\title{
Space weather: the solar perspective
}

\section{An update to Schwenn (2006)}

\author{
Manuela Temmer $^{1}$
}

Received: 18 August 2020 / Accepted: 15 April 2021 / Published online: 29 June 2021

(C) The Author(s) 2021

\begin{abstract}
The Sun, as an active star, is the driver of energetic phenomena that structure interplanetary space and affect planetary atmospheres. The effects of Space Weather on Earth and the solar system is of increasing importance as human spaceflight is preparing for lunar and Mars missions. This review is focusing on the solar perspective of the Space Weather relevant phenomena, coronal mass ejections (CMEs), flares, solar energetic particles (SEPs), and solar wind stream interaction regions (SIR). With the advent of the STEREO mission (launched in 2006), literally, new perspectives were provided that enabled for the first time to study coronal structures and the evolution of activity phenomena in three dimensions. New imaging capabilities, covering the entire Sun-Earth distance range, allowed to seamlessly connect CMEs and their interplanetary counterparts measured in-situ (so called ICMEs). This vastly increased our knowledge and understanding of the dynamics of interplanetary space due to solar activity and fostered the development of Space Weather forecasting models. Moreover, we are facing challenging times gathering new data from two extraordinary missions, NASA's Parker Solar Probe (launched in 2018) and ESA's Solar Orbiter (launched in 2020), that will in the near future provide more detailed insight into the solar wind evolution and image CMEs from view points never approached before. The current review builds upon the Living Reviews article by Schwenn from 2006, updating on the Space Weather relevant CME-flareSEP phenomena from the solar perspective, as observed from multiple viewpoints and their concomitant solar surface signatures.
\end{abstract}

Keywords Space weather - Solar physics - CMEs - Flares - SEPs - Dynamic corona $\cdot$ Magnetic field

Manuela Temmer

manuela.temmer@uni-graz.at

1 Institute of Physics, University of Graz, Graz, Austria 


\section{Contents}

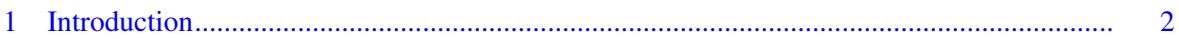

2 Space weather ................................................................................................. 7

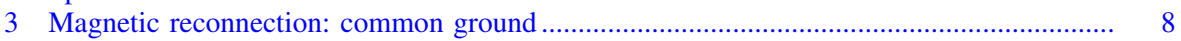

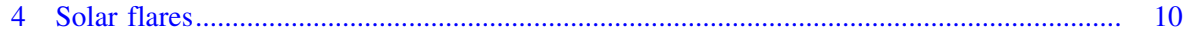

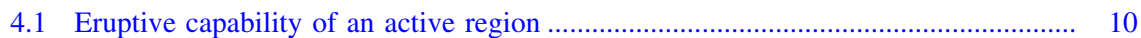

4.2 Eruptive solar flares: general characteristics ........................................................... 11

5 Coronal mass ejections (CMEs) ................................................................................ 14

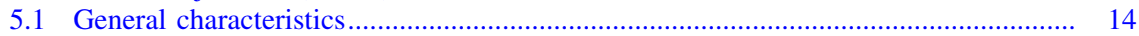

5.2 CME early evolution ............................................................................... 16

5.2.1 Shock formation, radio bursts, and relation to SEPs.................................... 19

5.2.2 Stealth CMEs....................................................................................... 22

5.3 Advantages due to multi-viewpoint observations ................................................ 22

6 Interplanetary counterparts of CMEs: ICMEs..................................................................... 27

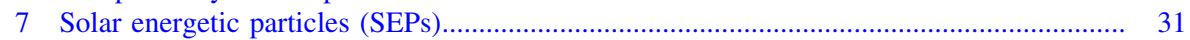

7.1 General characteristics ...................................................................................... 31

7.2 SEPs observed from multiple viewpoints............................................................ 35

8 Energy budget between flares, CMEs, and SEPs ............................................................ 37

9 Structuring of interplanetary space: the solar wind ........................................................ 38

9.1 General characteristics ................................................................................... 38

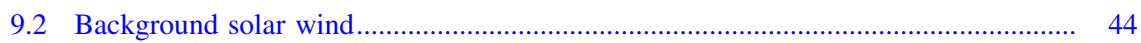

9.3 Solar wind structures affecting CME and SEP evolution..................................... 45

9.4 Preconditioning of interplanetary space............................................................... 46

10 The chain of action on the example of the September 2017 events ................................ 48

11 Space weather forecasting models .......................................................................... 53

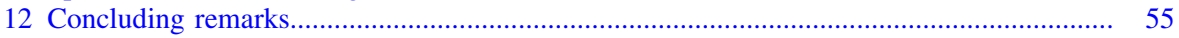

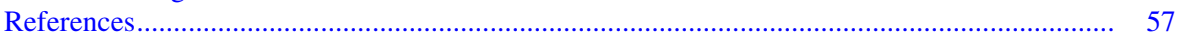

\section{Introduction}

Our Sun is an active star and as such undergoes cyclic variations, which are related to more or less frequently occurring activity phenomena observed at the solar surface. High energetic activity phenomena, produced due to changes in the Sun's magnetic field, propagate through our solar system where they interact with the planet's atmospheres. At Earth, these interactions are well documented and known to cause geomagnetic disturbances having consequences for modern society. The influence by the Sun on our solar system is termed Space Weather. Therefore, solar activity needs to be permanently monitored from space and ground in order to assess times of increased influence. International space agencies created programs, such as ESA Space Situational Awareness (SSA) or NASA Living With a Star (LWS) (cf. Fig. 1), to enhance Space Weather awareness and with that support and fund on a long-term basis fundamental research and development of Space Weather forecasting tools.

This review article focuses on the following Space Weather phenomena:

1. Coronal mass ejections

2. Flares 


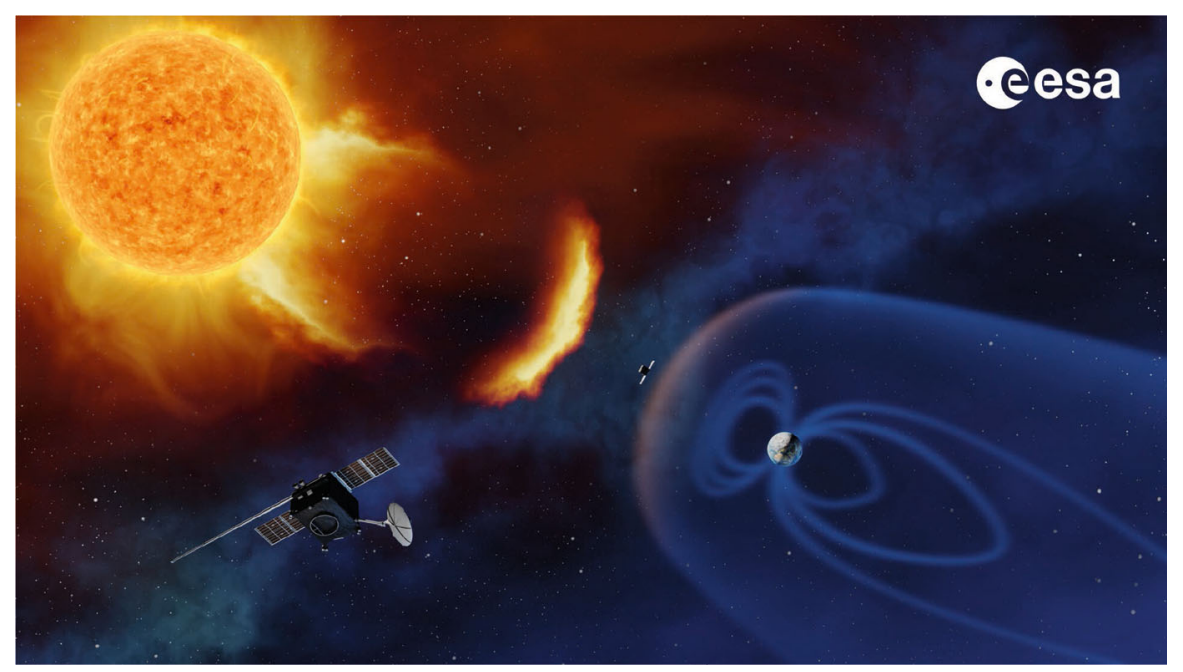

Fig. 1 Solar activity phenomena (depicted here as CME) affect Earth and near-Earth space and therefore, need to be permanently monitored. Space Weather forecasting is of global interest and funded by international agencies. In the near future, satellites will observe the Sun and its dynamic phenomena from different viewpoints, such as a combined L1 and L5 position. Image courtesy: ESA

\section{Solar Energetic Particles}

4. Solar wind stream interaction regions

To properly describe these phenomena from the solar perspective, a number of processes need to be understood, such as active region and magnetic field evolution, energy build-up and release, as well as the global structuring of inner heliospheric space. Space Weather is a topic of broad interest and sustains an exciting and wealthy interdisciplinary research community. ${ }^{1}$ With that it fosters information and knowledge exchange between international research groups on solar-, heliosphericand geo-space (Sun-to-impact disciplines) in order to enhance scientific knowledge for improving existing and developing new models for Space Weather forecasting.

Coronal mass ejections (CMEs) are a rather recent phenomenon, discovered just about 50 years ago, but in the meantime are known as the main drivers of the most severe Space Weather disturbances (see e.g., Howard 2006; Gopalswamy 2016). They are huge structures that manifest themselves within some tens of minutes as clouds of magnetized plasma impulsively expelled from the Sun and subsequently propagating into interplanetary space (see e.g., Forbes 2000). CMEs arise from usually complex and closed magnetic field structures in equilibrium that is disrupted due to some instability causing its eruption (e.g., emerging magnetic flux, remote reconfiguration of large scale magnetic field, or field rotation; see e.g., Török et al. 2013; Schmieder et al. 2015; Green et al. 2018). Instabilities in the solar magnetic

\footnotetext{
${ }^{1}$ For example, the SCOSTEP effort that resulted in excellent publications via CAWSES http://www. terrapub.co.jp/onlineproceedings/ste/CAWSES2007/index.html, the VarSITI programs (e.g., ISEST Zhang et al. 2018, see http://www.varsiti.org) or the international Space Weather Action Teams, iSWAT, where interdisciplinary groups gather together under https://www.iswat-cospar.org.
} 


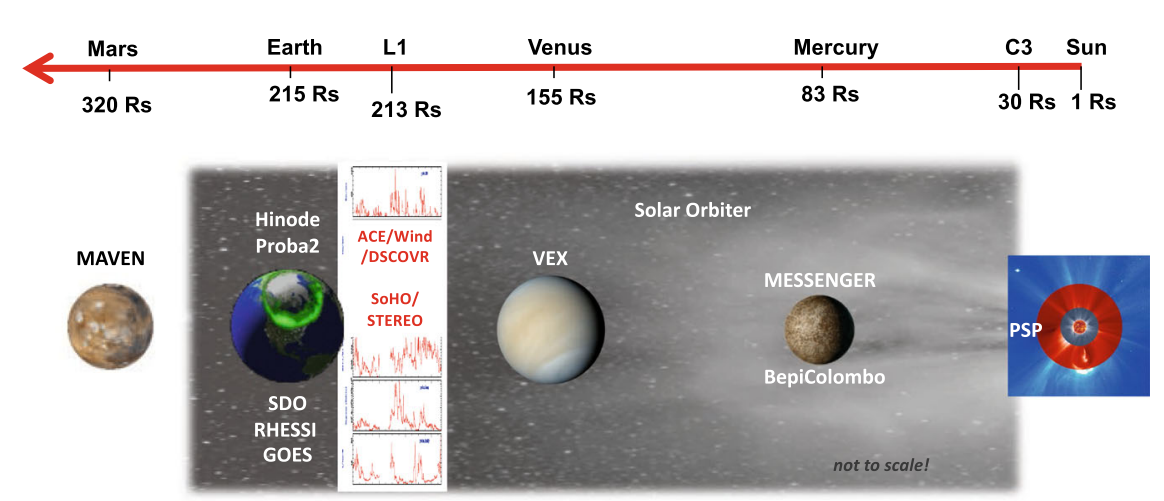

Fig. 2 Current and past space missions carrying instruments for gathering remote sensing image data and in-situ plasma and magnetic field measurements. The majority of spacecraft is located in the ecliptic plane orbiting planets or at the Lagrangian point L1. The coronagraph field of view of the SoHO/LASCO instrument $\mathrm{C} 3$ covers 30 solar radii. The background white-light image is taken from STEREO/HI1+2 data covering about 90 degrees in the ecliptic. Not to scale

field and their occurrence frequency are modulated by the 11-year activity cycle of the Sun. The most strong CME events may propagate the $1 \mathrm{AU}$ distance within a day (e.g., Cliver et al. 1990; Gopalswamy et al. 2005a; Liu et al. 2014). Less strong events, on average, propagate the same distance in up to 4 days (see e.g., Shanmugaraju and Vršnak 2014). CMEs may be linked to large geomagnetic disturbances, due to shock compression and reconnection with the Earth's magnetic field. They may lead to ionospheric and geomagnetically-induced currents (see e.g., Pirjola et al. 2005). Usually the most severe geomagnetic storms are caused by fast and massive CMEs, erupting from the central region of the visible solar disk and carrying a strong southward magnetic field component that reconnects with the Earth's magnetic field (see e.g., Pulkkinen 2007). Consequently, CMEs are a major topic of solar and Space Weather research.

The power for making a CME energetic (i.e., being fast and wide) undoubtedly stems from the free magnetic energy which is released as consequence of magnetic reconnection processes. Magnetic reconnection enables to impulsively drive plasma and to accelerate particles to high energies causing on the one hand flare emission, which is observed in the solar atmosphere, and on the other hand solar energetic particles (SEPs), which are measured in interplanetary space. Energetic particles from strong SEP events may reach almost speed of light and travel the $1 \mathrm{AU}$ distance within about $10 \mathrm{~min}$. High energy SEP events (about $1 \mathrm{GeV}$ ) may lead to enhanced proton fluxes even at ground level. Hence, most intense events can endanger life and technology on Earth and in space. Further consequences of CMEs and SEPs are disruptions of satellite operations, radio communications and ground power systems (e.g., Bothmer et al. 2007). Unlike CMEs, having lead times of some tens of hours between first observational signatures and impact at Earth, flares and SEP events occur and impact almost simultaneously (see e.g., Lugaz et al. 2017b; Cairns et al. 2018; Malandraki and Crosby 2018). Accordingly, to predict the 


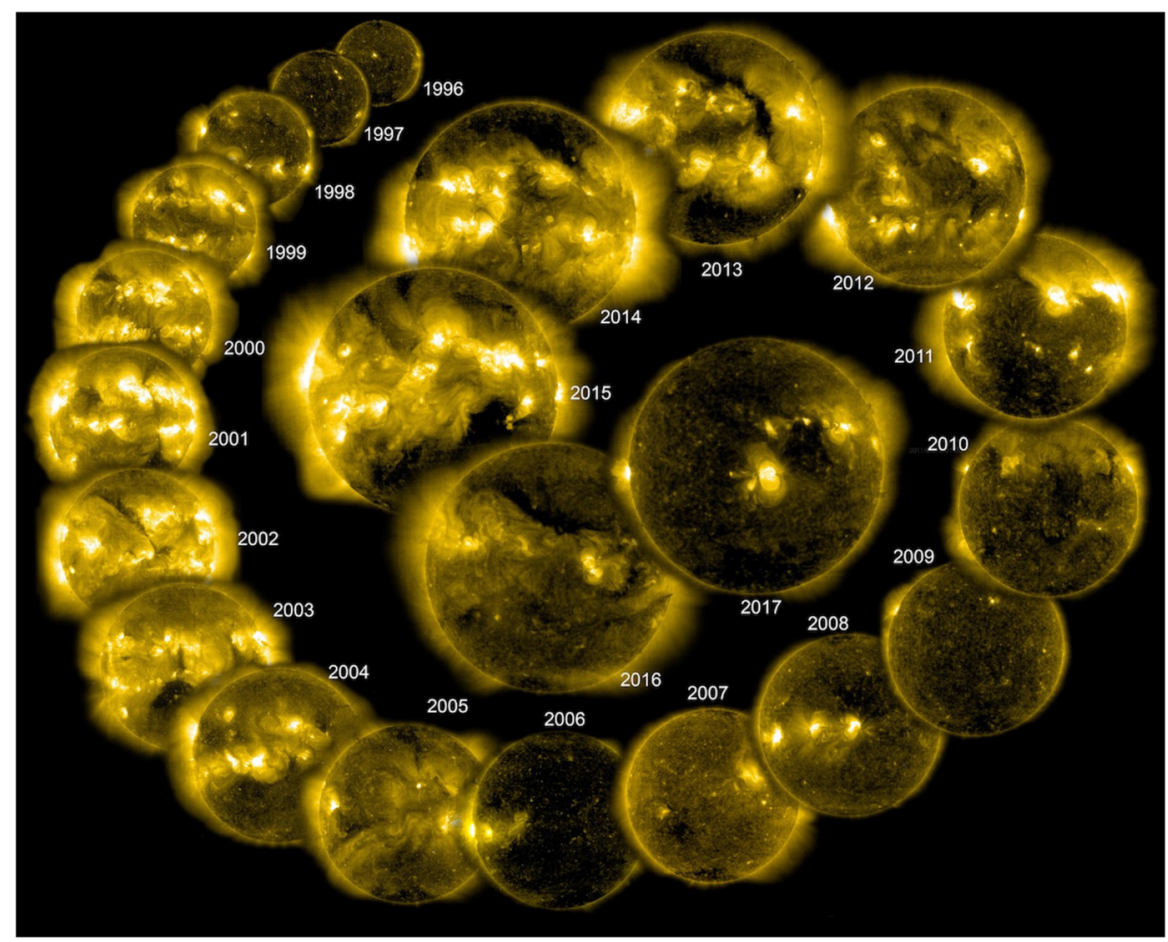

Fig. 3 Each image shown here is a snapshot of the Sun taken every spring with the SOHO Extreme ultraviolet Imaging Telescope (EIT) in the $284 \AA$ 政 wavelength range. It shows the variations of the solar activity in terms of increasing and decreasing number of bright active regions visible in the corona. Courtesy: NASA/ESA

occurrence of flares and SEPs one needs to predict the instabilities leading to the onset of magnetic reconnection processes, one of the big challenges in solar physics.

The continuous solar wind flow in a quiet state (usually termed background solar wind) is represented by an alternation of slow and fast solar wind streams that interact and form stream interaction regions (SIRs). If steady in their existence and persisting over more than one solar rotation, they are called co-rotating interaction regions (CIRs). During times of low solar activity, Space Weather is dominated by CIR induced storms (Tsurutani et al. 2006). Different flow speeds of the background solar wind also change the propagation behavior of CMEs in interplanetary space. This has consequences on the CME transit time and impact speed at planetary atmospheres (drag force; see Gopalswamy et al. 2000; Vršnak 2001; Cargill 2004; Vršnak 2006). Moreover, CMEs disrupt the continuous outflow of the solar wind and reconfigure the magnetic field on large spatial and short temporal scales altering the background solar wind. For Space Weather and CME modeling/forecasting purposes, these ever changing conditions in interplanetary space are very challenging to tackle.

For comprehensive investigations a rich source of observational data is currently available from many different instruments located at multiple viewpoints and 
different radial distances (see Fig. 2). In Earth orbit current operational missions are e.g., GOES (Geostationary Operational Environmental Satellite), SDO (SDO: Pesnell et al. 2012), Proba-2 (Santandrea et al. 2013), located at L1-1.5 million $\mathrm{km}$ upstream of Earth-there is the Solar and Heliospheric Observatory (SoHO: Domingo et al. 1995), the Advanced Composition Explorer (ACE: Stone et al. 1998), the WIND spacecraft Ogilvie et al. (1995), and DSCOVR (Burt and Smith 2012). At $\sim 1$ AU with variable longitudinal angles from Earth, there is the Solar TErrestrial RElations Observatory (STEREO: Kaiser et al. 2008) consisting of two identical spacecraft named STEREO-Ahead and STEREO-Behind (lost signal end of 2014). The combination of remote sensing image data and in-situ measurements is found to be optimal for enhancing our knowledge about the physics of Space Weather phenomena. For better understanding large eruptive activity phenomena, multi-viewpoint and multi-wavelength data are exploited (e.g., combined L1, STEREO as well as ground-based instruments). The various available data from spacecraft orbiting around planets [e.g., VEX (2006-2014), MESSENGER (2011-2015), MAVEN (2014-), BepiColombo (2018-)] also enable to analyze the evolution of Space Weather phenomena as function of distance and longitude.

A flagship of international collaboration and boost for Space Weather research, is SoHO which now achieved 25 years in space. Figure 3 shows SOHO/EIT (Delaboudinière et al. 1995) EUV image data covering the variations of the solar corona over a full magnetic solar cycle (Hale cycle). Long-term observations are of utmost importance for monitoring and learning about the interaction processes of solar activity phenomena with Earth and other planets as well as for improving our capabilities in Space Weather forecasting. Most recent and unprecedented missions are Parker Solar Probe, launched in August 2018 (Fox et al. 2016), and Solar Orbiter, launched in February 2020 (Müller et al. 2020) having on-board imaging and in-situ facilities with the goal to approach the Sun as close as never before ( $\sim 0.05 \mathrm{AU}$ and $\sim 0.3 \mathrm{AU})$ and investigating the Sun out of the ecliptic $(\sim 30$ degrees). To support space missions and for providing valuable complementary data, we must not forget the importance of ground based observatories that observe the Sun over broad wavelength and energy ranges allied in international networks such as the Global high-resolution $\mathrm{H} \alpha$ network, ${ }^{2}$ the Global Oscillation Network Group, ${ }^{3}$ the database for high-resolution Neutron Monitor measurements, ${ }^{4}$ muon telescope networks, or the Worldwide Interplanetary Scintillation Stations Network. ${ }^{5}$

From the derived research results based on the observational data, over the past years a plethora of models could be developed for predicting Space Weather and their geomagnetic effects. The permanent monitoring of the Sun and provision of

\footnotetext{
${ }^{2}$ http://bbso.njit.edu/Research/Halpha/.

3 https://gong.nso.edu.

${ }^{4}$ For example, http://www01.nmdb.eu/nest/search.php.

5 http://helios.mexart.unam.mx/pruebas/wipss/index.html.
} 
data in almost real-time enabled to apply those results and even to install operational services that produce forecasts mostly in an automatic manner (e.g., facilitated by ESA/SSA; ${ }^{6}$ NASA/CCMC; ${ }^{7}$ NOAA/SWPC ${ }^{8}$ ). However, the operational services also clearly demonstrated the limitations in the forecasting accuracy as on average the errors are large and get worse with increasing solar activity. This is mainly due to the large uncertainties coming from the model input, namely observational parameters at or close to the Sun. It also reveals the complexity of the interplay between the different driving agents of Space Weather, that makes it difficult to fully capture the physics behind and to improve models. Reliable Space Weather forecasting is still in its infancy.

\section{Space weather}

From the historical perspective, the so-called "Carrington-event" from September 1,1859 is the reference event for referring to extreme Space Weather and with that the beginning of Space Weather research (see also Schwenn 2006). At that time only optical observations of the solar surface were performed and the observed emitted radiation in white-light for that event showed impressively the vast amount of energy that was distributed to the dense lower atmospheric layers of the Sun where it heated the photosphere. At Earth, the associated geomagnetic effects were observed in terms of aurorae occurring from high to low latitudes (e.g., Honolulu at 20 degrees northern latitude) and ground-induced currents in telegraph wires (see Eastwood et al. 2017). The associated SEP event is thought to be about twice as large as the huge SEP events from July 1959, November 1960, or August 1972 (Cliver and Dietrich 2013). Only several years after the Carrington event, the usage of spectroscopes enabled to regularly observe prominence eruptions revealing the dynamic changes of the solar corona and material ejections with speeds exceeding hundreds of km/s (Tandberg-Hanssen 1995). The continuous monitoring of the Sun was intensified in the 1940s, when solar observations in radio, white-light and in the $\mathrm{H} \alpha$ wavelength range were performed. At that time also galactic cosmic rays were studied and found that they are anti-correlated with solar activity (so-called Forbush decrease, measured as sudden drop in the cosmic ray flux due to interplanetary disturbances; see also Cane 2000). In the early 1960s, magnetic structures driving shocks were inferred from observations in the metric radio observations and geomagnetic storm sudden commencements (Gold 1962; Fokker 1963). The transient events with mass moving through the solar corona and actually leaving the Sun, i.e., CMEs, that were associated with the prominence/filament eruptions were discovered only in the early 1970s with the advent of the space era (see Tousey 1971; MacQueen et al. 1974). Recent reviews on the history of prominences and their role in Space Weather can be found in (Vial and Engvold 2015; Gopalswamy 2016, and references therein). While most of the extreme space weather events

\footnotetext{
6 http://swe.ssa.esa.int.

7 https://ccmc.gsfc.nasa.gov.

${ }^{8}$ https://www.swpc.noaa.gov.
} 
happen during the solar cycle maximum phase, occasionally strong geoeffective events may occur close to the solar cycle minima and also during weak solar activity, provided there are appropriate source regions on the Sun (see also e.g., Vennerstrom et al. 2016; Hayakawa et al. 2020). For more details about the solar cycle see the Living Review by Hathaway (2010).

Nowadays, a wealth of space and ground-based instruments are available, delivering valuable observational data, as well as modeling facilities. This enables to study in rich detail the manifold processes related to Space Weather events and to better understand the physics behind. To forecast the geomagnetic effects of an impacting disturbance at Earth (e.g., by the $\mathrm{Dst}^{9}$ or $\mathrm{Kp}$ index ${ }^{10}$ ), the most common parameters we need to know in advance-and various combinations of these-are the amplitude/orientation and variation of the north-south component of the interplanetary magnetic field $\left(B_{\mathrm{z}}\right)$, speed $(v)$, and density $(n)$. Especially, the electric field $v B_{\mathrm{s}}\left(B_{\mathrm{s}}=B_{z}<0\right)$ is found to show a high correlation with the Dst storm index (see e.g., Baker et al. 1981; Wu and Lepping 2002; Gopalswamy et al. 2008a). For details on the geomagnetic effects of Space Weather phenomena as described here, see the Living Review by Pulkkinen (2007).

The Space Weather "chain of action" from the solar perspective is described best by the recent example of the multiple Space Weather events that occurred in September 2017 (see Sect. 10). But before that, we elaborate the physical basis.

\section{Magnetic reconnection: common ground}

The commonality that unites everything and yet produces such different dynamic phenomena is magnetic reconnection and the release of free magnetic energy. This leads to particle acceleration, heating, waves, etc. and to a restructuring of the (local) magnetic field in the corona by newly connecting different magnetic regimes and with that changing magnetic pressure gradients. Especially the latter shows to affect the solar corona globally.

In order to derive a complete picture about Space Weather, we first need to understand the interrelation between these many individual processes starting at the Sun. This covers a cascade of small and large scale phenomena varying over different time scales. The primary source of Space Weather producing phenomena, i.e., CMEs-flares-SEPs (note that in the following eruptive phenomena are considered and not stealth CMEs), are active regions representing the centers of strong magnetic field and energy (more details on the evolution of active regions, see the Living Reviews by van Driel-Gesztelyi and Green 2015; Toriumi and Wang 2019). However, in detail the energy build-up and release processes are not well understood. The key-driver certainly is the magnetic field configuration below the visible surface (photosphere), that cannot be directly observed and characterized for giving reliable predictions of its status and further development. The lack of magnetic field information is also given in the upper atmospheric layers. There are

\footnotetext{
9 The disturbance storm time (Dst) index monitors variations in the Earth's equatorial ring current.

10 The planetary $\mathrm{K}$ index (Kp) monitors variations in the horizontal component of the Earth's magnetic field.
} 

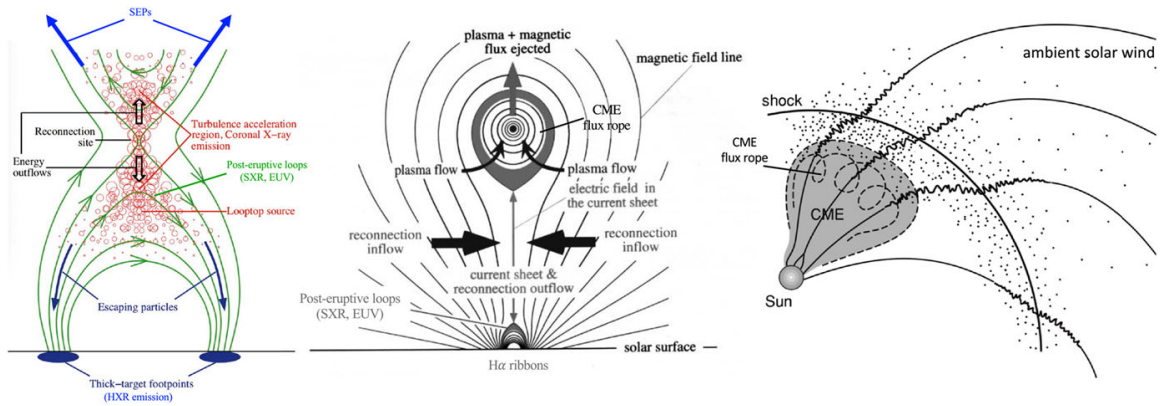

Fig. 4 Left: Stochastic acceleration model for solar flares. Magnetic field lines (green) and turbulent plasma or plasma waves (red circles) generated during magnetic reconnection. Blue arrows and areas mark accelerated particles impinging on the lower denser chromosphere where they produce Bremsstrahlung and on the upside may escape to interplanetary space where they are detected as SEPs (adapted from Petrosian and Liu 2004; Vlahos et al. 2019). Middle: CME-flux rope configuration in the classical scenario (CSHKP) covering also the post eruptive arcade usually observed in SXR and EUV wavelength range (adapted from Lin and Forbes 2000); Right: CME flux rope acting as driver of a bow shock (black arc) may accelerate SEPs (black dots) in the corona or heliosphere via diffusive shock acceleration (adapted from Mikić and Lee 2006)

currently no instruments enabling measurements of the magnetic field in the corona, hence, we need to rely on models simulating the coronal and, further out, interplanetary magnetic field (see, e.g., the Living Review by Gombosi et al. 2018, on coronal and solar wind MHD modeling ). While active regions are characterized by closed magnetic field, coronal holes cover mainly open magnetic field from which high speed solar wind streams emerge. They structure interplanetary space and set the coupling processes between continuous solar wind flow and transient events. To better understand the propagation behavior of transient events, we also need to study the evolution and characteristics of the solar wind flow, and hence, the interplay between open and closed magnetic field.

Figure 4 sketches three different time steps in the evolution of an eruptive flare event, causing a CME and SEPs, as a consequence of magnetic reconnection (see Petrosian and Liu 2004; Lin and Forbes 2000; Mikić and Lee 2006). The left panel of Fig. 4 focuses on the early evolution stage of the eruptive event, introducing stochastic acceleration processes causing high energetic particles to precipitate along magnetic field lines towards and away from the Sun. Flare emission is observed on the solar surface due to the acceleration of particles towards the Sun. Particles that escape into interplanetary space along the newly opened magnetic field, produce SEPs. The middle panel of Fig. 4 shows the creation of the CME body, i.e., the production of a closed magnetic field structure (flux rope), as well as the related post-eruptive arcade which is formed below. The exact acceleration mechanism(s) of SEPs is still an open issue, hence, cartoons as shown here usually present both possible driving agents, the flare and the CME shock. To complete the picture for a flare-CME-SEP event, the right panel of Fig. 4 depicts the interplanetary magnetic field and its behavior which differs from the typical Parker spiral orientation due to the propagating CME shock component causing SEP acceleration in interplanetary space. The deviation of the interplanetary magnetic 
field from the nominal Parker spiral is an important issue when dealing with magnetic connectivity for studying SEPs and propagation behavior of CMEs.

In the following, we will discuss in more detail the characteristics of the different manifestations occurring in an eruptive flare event.

\section{Solar flares}

\subsection{Eruptive capability of an active region}

Active regions may be classified either by the morphology of an active region using the McIntosh classification (McIntosh 1990) or the magnetic structure using Hale's/ Künzel's classification (Künzel 1960). Due to the emergence of magnetic flux the degree of complexity in the magnetic field of an active region grows, which increases the likeliness to create strong flares and CMEs (e.g., Sammis et al. 2000; Toriumi et al. 2017). The probability that an X-class flare is related to a CME is found to be larger than $80 \%$ (Yashiro et al. 2006), however, there are well observed exceptions reported. So-called confined flares are neither accompanied by a CME nor a filament eruption (e.g., Moore et al. 2001). Their special magnetic field configuration allows particle acceleration (observed as flare), but they do not escape into interplanetary space and, hence, do not produce SEPs (Gopalswamy et al. 2009). Therefore, confined flares may produce strong X-ray emission but, presumably due to a strong bipolar overlying coronal magnetic field configuration, are not related to the opening of the large-scale magnetic field (e.g., Wang and Zhang 2007; Sun et al. 2015; Thalmann et al. 2015). The electromagnetic radiation of confined flares can still instantaneously cause sudden changes in the ionospheric electron density profile (disturbing radio wave communication or navigation), also known as solar flare effect or geomagnetic crochets (Campbell 2003) but occurring rather rarely. However, confined flares are also potential candidates for false Space Weather alerts in terms of an erroneous forecast of geomagnetic effects due to the magnetic ejecta that would have arrived tens of hours later at Earth.

Therefore, the manifestation of the eruptive capability of an active region is one of the prime targets for prospective forecasting of SEPs and CMEs. For example, the length of the main polarity inversion line of an active region or the magnetic shear and its sigmoidal morphology, is obtained to be highly indicative of the potential to open large scale magnetic field and to produce CMEs and SEPs (e.g., Canfield et al. 1999). Studies also showed that active regions, for which the polarity inversion line quickly changes with height into a potential field configuration, are more favorable for producing non-eruptive events (Baumgartner et al. 2018). Likewise, the decay index of the horizontal magnetic field (ratio of the magnetic flux in the lower corona to that in the higher corona) is found to be lower for failed eruptions compared to that for full eruptions (cf. Török and Kliem 2005; Fan and Gibson 2007; Guo et al. 2010; Olmedo and Zhang 2010).

For more details on the issue of flare-productive active regions, I refer to the Living Review by Toriumi and Wang (2019). See also Forbes (2000), Webb and 
Fig. 5 Flare-CME-SEP relation in time. The onset of the solar flare is indicated by the vertical red line. The grey shaded area marks the time difference between flare start and SEP flux increase for $\mathrm{MeV}$ energies. Image reproduced with permission from Anastasiadis et al. (2019), copyright by the authors, who adapted it from Miroshnichenko (2003)

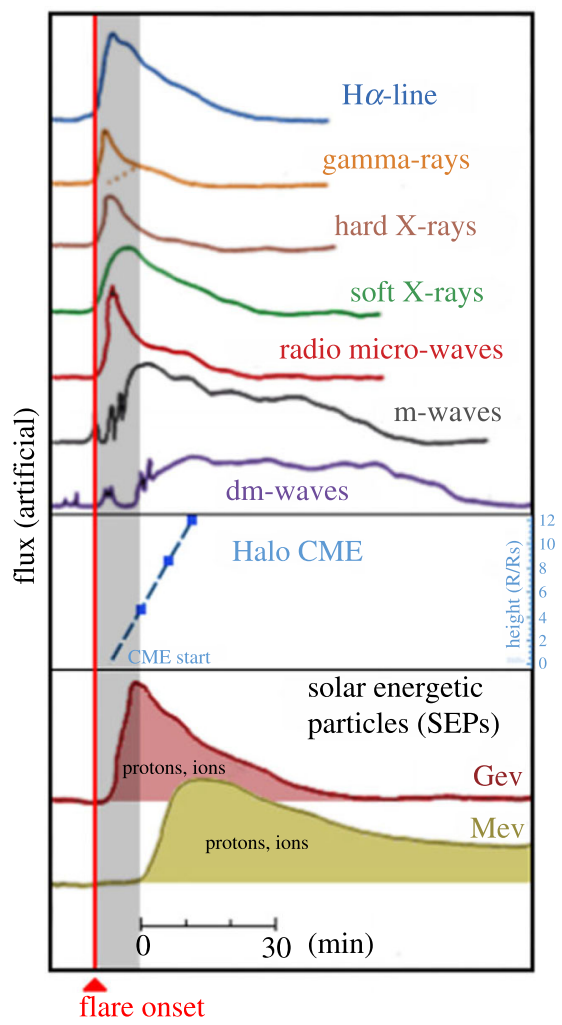

Howard (2012), Parenti (2014), or Chen (2017) for a more theoretical approach on that issue.

\subsection{Eruptive solar flares: general characteristics}

Flares are observed to release a huge amount of energy from $10^{19}$ up to $10^{32} \mathrm{erg}$ over a timescale of hours. ${ }^{11}$ With the advent of modern ground-based and spaceborne instruments, our small optical window was massively enlarged and it is now well-known that this energy is radiated over the entire electromagnetic spectrum from decameter radio waves to gamma-rays beyond $1 \mathrm{GeV}$. Figure 5 depicts the temporal relation between flare emission, observed in different wavelength ranges, CME kinematics and SEP flux profiles in the $\mathrm{GeV}$ and $\mathrm{MeV}$ energy range. The flare activity profile consists of a so-called pre-flare phase, showing thermal emission in SXR and EUV, as well as $\mathrm{H} \alpha$ kernel brightenings. If related to a filament eruption, this phase partly coincides with the slow rise phase of the filament. ${ }^{12}$ This is

\footnotetext{
11 An automatically updated list of flares is available under http://www.lmsal.com/solarsoft/latest_ events/ or https://www.solarmonitor.org.

12 Filament detection and eruption catalogues can be found e.g., under http://cesar.kso.ac.at/sn_iv/ filaments.php or http://aia.cfa.harvard.edu/filament/.
} 
followed by the impulsive flare phase during which most of the energy is released and non-thermal emission in terms of hard X-ray (HXR) footpoints appears due to particles accelerating out of the localized reconnection area and bombarding the denser chromosphere where they emit Bremsstrahlung (for a review on solar flare observations see e.g., Fletcher et al. 2011). At this point also the CME body forms as consequence of the closing of the magnetic field lines in the upper part of the reconnection area revealing a flux rope structure (note that the most compelling argument for an already existing flux rope is actually a filament). As the flare emission increases also the SEP flux in the GeV energy range starts to rise. After the flare reaches a maximum in intensity, the decay phase is observed during which the intensity level goes back to the background level from before the flare start. The exact timing of the rise and decay phase is dependent on the energy release and the energy range in which the flare is observed which is known as the so-called Neupert effect (the HXR flux rise phase time profile corresponds to the derivative of the SXR flux time profile; see Neupert 1968). The last phase may have a duration of several hours or longer. During that phase also post-eruptive arcades (or loops) start to form, that may still grow over 2-20 hours. The growth of the post-eruptive arcade is hinting towards an ongoing reconnecting process, which is not energetic enough to produce a significant emission in EUV or SXR (see e.g., Tripathi et al. 2004). For

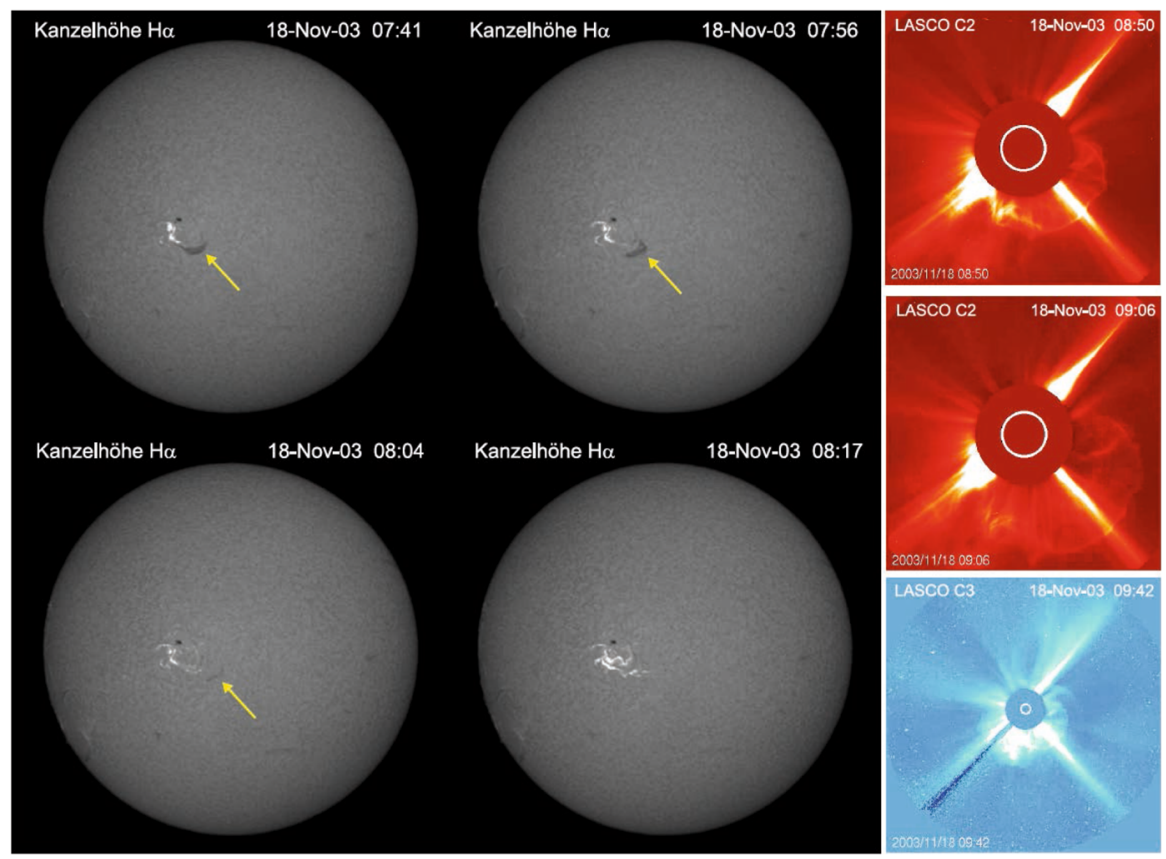

Fig. 6 Global flare evolution and relation to CME from the November 18, 2003 event. Left panels: $\mathrm{H} \alpha$ filtergrams from the Kanzelhöhe Solar Observatory (Austria). The associated erupting filament is indicated by arrows. Right panels: Temporal evolution of the CME in coronagraph images from $\mathrm{SOHO} /$ LASCO. Image reproduced with permission from Möstl et al. (2008), copyright by the authors 
more details on the global properties of solar flares, I refer to the review by Hudson (2011).

Figure 6 shows the temporal evolution of a flare and erupting filament observed in $\mathrm{H} \alpha$ and the associated $\mathrm{CME}$ observed in white-light coronagraph image data. The event is classified in the emitted SXR flux as GOES M3.9 flare (corresponding to the measured power of $3.6 \times 10^{-5} \mathrm{~W} / \mathrm{m}^{2}$ ) which occurred on November 18, 2003 in a magnetically complex $\beta \gamma$ active region. The associated CME caused two days later one of the strongest geomagnetic storms of solar cycle 23 having a minimum Dst value of $-472 \mathrm{nT}$ (Gopalswamy et al. 2005b). Inspecting the time stamps on the image data of that event, about one hour after the appearance of the flare signatures, the CME became visible in the coronagraph. The filament started to rise some tens of minutes before the flare emission occurred.

The orientation of the magnetic structure, especially of the $B_{\mathrm{z}}$ component, of an ICME is key to forecast its geoeffectiveness and poses the Holy Grail of Space Weather research. Knowing the flux rope orientation already at the Sun could provide information on the impact of CMEs early in advance, hence, as soon as they erupt or even before. While the handedness of flux ropes can be well observed from in-situ measurements (Bothmer and Schwenn 1998; Mulligan et al. 1998), on the
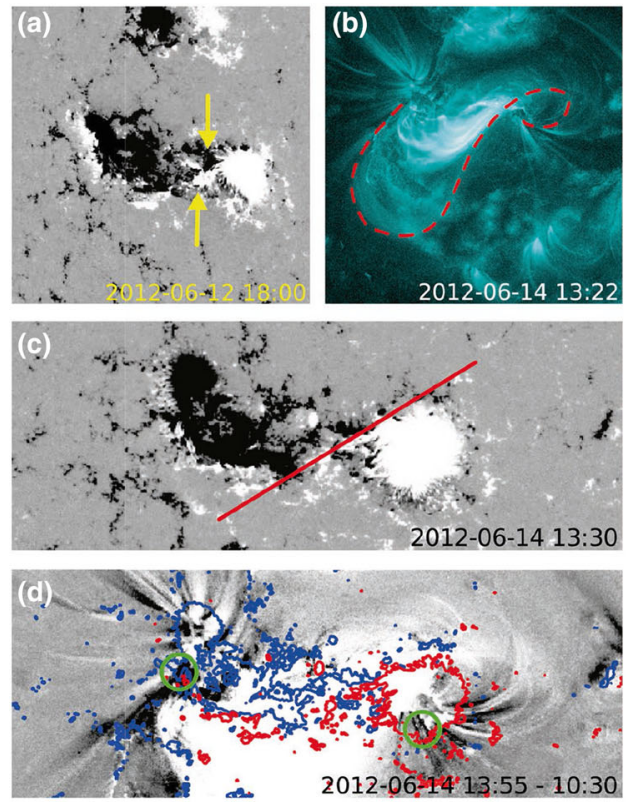

(e)

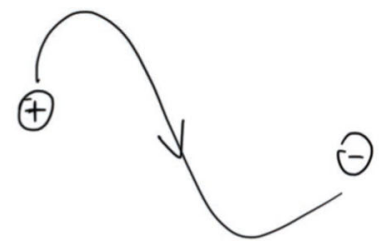

left-handed

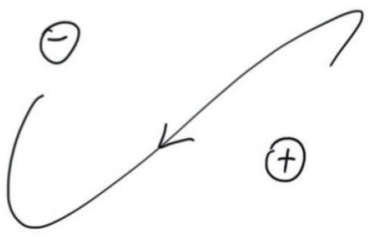

right-handed

Fig. 7 a SDO/HMI data from June 14, 2012 showing the magnetic tongues of the erupting active region revealing a positive chirality. b Forward-S sigmoidal structure from the coronal loops observed by SDO/ AIA $131 \AA$, indicating a right-handed flux rope. c SDO/HMI magnetogram showing the approximated polarity inversion line (red line). d Base-difference SDO/AIA $131 \AA$ image overlaid with the HMI magnetogram contours saturated at $\pm 200 \mathrm{G}$ (blue = negative polarity; red = positive polarity). The dimming regions indicating the flux rope footpoints are marked by green circles. e The cartoon shows the handedness inferred from the magnetic field and sigmoidal structures or orientation of post eruptive loops. Images (a-d) reproduced with permission from Palmerio et al. (2018), copyright by AGU 
Sun observational proxies need to be used. Figure 7 shows several surface signatures from which the magnetic helicity (sense of twist of the flux rope: righthanded or left-handed) can be inferred. Typically, in EUV observations these are sigmoidal structures (S- or reverse $\mathrm{S}$-shaped) or post-eruptive arcades (skewness of EUV loops and polarity of the underlying magnetic field), in $\mathrm{H} \alpha$ the fine structures of filaments are used (orientations of barbs) or statistical relations like the hemispheric helicity rule (see Wang 2013). However, strong coronal channeling, latitudinal and also longitudinal deflection and/or rotation, that the magnetic component of the CME undergoes as it evolves through the low solar corona, may change those parameters as shown in various studies by e.g., Shen et al. (2011), Gui et al. (2011), Bosman et al. (2012), Panasenco et al. (2013), Wang et al. (2014), Kay and Opher (2015), Möstl et al. (2015), or Heinemann et al. (2019). Recent approaches in ICME $B_{z}$ forecasting can be found in, e.g., Savani et al. (2015), Palmerio et al. (2017), or Kay and Gopalswamy (2017).

For more details about the energetics and dynamics of solar flares, I refer to the Living Review by Benz (2017) and for the magnetohydrodynamic processes in active regions responsible for producing a flare to the Living Review by Shibata and Magara (2011).

\section{Coronal mass ejections (CMEs)}

\subsection{General characteristics}

CMEs are optically thin large-scale objects, that quickly expand, and are traditionally observed in white-light as enhanced intensity structures. The intensity increase is due to photospheric light that is Thomson scattered off the electrons forming the CME body and integrated over the line-of-sight (Hundhausen 1993). Due to strong projection effects their apparent morphology greatly depends on the viewpoint and, hence, makes CMEs a rather tricky object to measure (see e.g., Burkepile et al. 2004; Cremades and Bothmer 2004).

By using coronagraphs, CMEs are visible with teardrop-like shapes that are characterized by multiple structures. Figure 8 shows SOHO/LASCO (Brueckner et al. 1995) coronagraph white-light images of two CMEs having different propagation directions. For the CME that leaves the Sun in a rather perpendicular angle to the observer (left panel of Fig. 8), the various CME structures are well visible. In general, we distinguish between the shock (yellow arrow) and CME body (green arrow) that are followed by some cavity created by the expanding magnetic flux rope (red arrow) and an increased brightness structure (orange arrow). Partly these structures are detected also from in-situ measurements for the interplanetary counterparts of CMEs (ICMEs; see Sect. 6). The increased brightness structure consists of prominence material (Vourlidas et al. 2013) or is suggested to appear due to a brightness increase of the two overlapping CME flanks (Howard et al. 2017). The sheath region behind the shock has less clear signatures in coronagraph images taken close to the Sun as it is generated later when the solar wind plasma gets piled-up in interplanetary space (see e.g., Kilpua et al. 2017; Salman et al. 

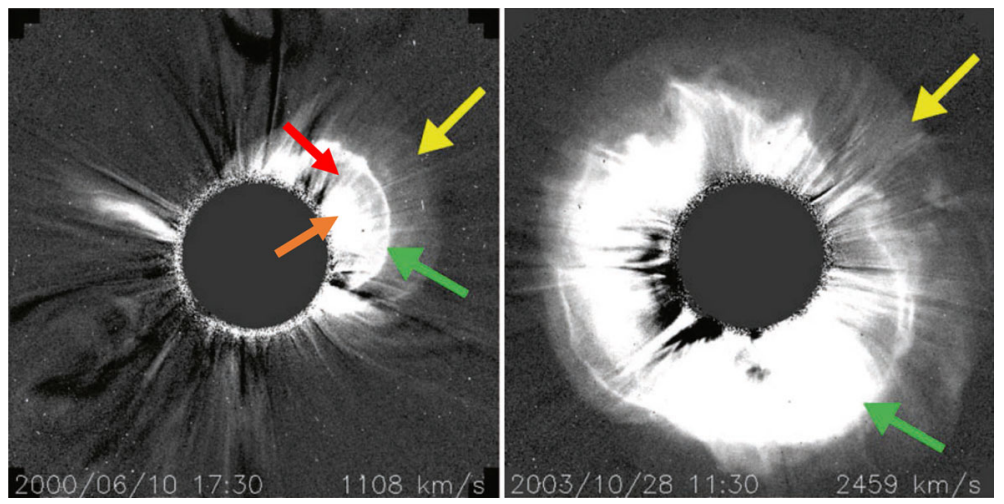

Fig. 8 LASCO CME excess mass images showing the expanding shock wave front (yellow arrow) and the CME leading edge density enhancement (green arrow) for two different events. For the CME propagating rather in the plane of sky (left panel), typical structures such as the cavity due to the expanding magnetic ejecta (red arrow) followed by some intensity enhancement (orange arrow) can be observed, that is less well visible for the halo CME (right panel). The projected LASCO CME speeds are given in the legend. Image adapted from Vourlidas et al. (2013), copyright by Springer

2020). For CMEs propagating in the line-of-sight towards or away from the observer (right panel of Fig. 8), the different structures are less well visible. As these CMEs are launched close to the central meridian of the observed disk, they most severely suffer from projection effects. Energetic ones are frequently observed as so-called halo CMEs, revealing extensive white-light signatures made of compressed plasma material surrounding the occulting disk of a coronagraph. For halo CMEs, evidence that the CME is actually moving towards the observer is given from the associated activities observed on the solar disk (such as filament eruptions, flare emission, dimming regions, or coronal wave signatures). Highly relevant for Space Weather, halo CMEs are of special interest and are diversely studied mostly by using single spacecraft data from the coronagraphs aboard SoHO.

Up to the distance of about 30 solar radii (LASCO/C3 field of view), statistical studies showed that CMEs undergo several phases in their dynamics. Before the actual launch a slow rising phase occurs (initiation phase), continued by the acceleration phase over which a rapid increase in speed is observed in the inner corona, that is followed by a rather smooth propagation phase as the CME leaves the Sun (Zhang and Dere 2006). On average, over the coronagraphic field of view, CME fronts reveal radial speeds in the range of $300-500 \mathrm{~km} / \mathrm{s}$ with maximum values observed up to $3000 \mathrm{~km} / \mathrm{s}$, accelerations of the order of $0.1-10 \mathrm{~km} / \mathrm{s}^{2}$, angular widths of about $30-65$ degrees and masses of $\sim 10^{14}-10^{16} \mathrm{~g}$ (e.g., Vourlidas et al. 2010; Lamy et al. 2019). The ratio in density between the CME body and surrounding solar wind decreases from $\sim 11$ at a distance of 15 solar radii to $\sim 6$ at 30 solar radii (Temmer et al. 2021). However, CMEs vary in their occurrence rate as well as in their characteristics over different solar cycles. While flare rates and their properties have not changed much over the past solar cycles, the CME properties for solar cycle 24 are significantly different as given in recent statistics (Lamy et al. 2019; Dagnew et al. 2020b). CMEs were found to be more numerous 


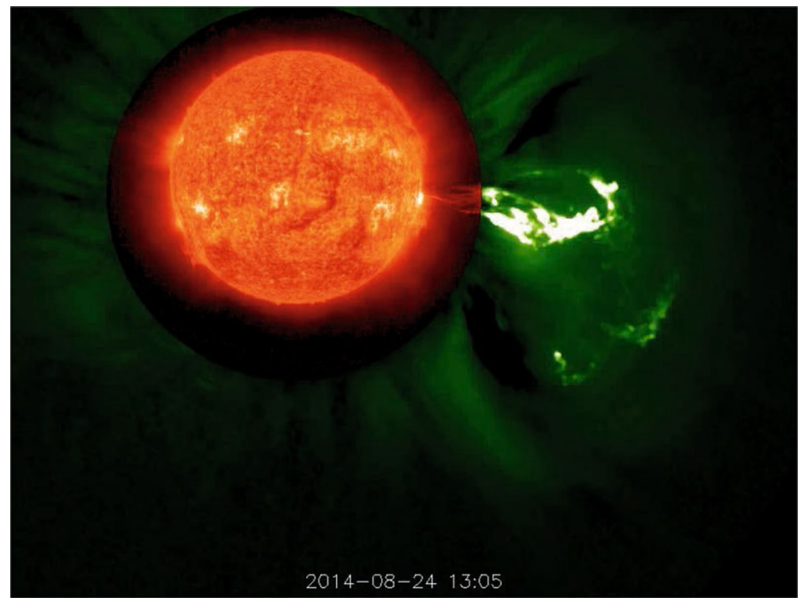

Fig. 9 STEREO-B observations of the CME from August 24, 2014. The images show combined EUVI (304 $\AA$ ) and COR1 image data. Filament plasma material is ejected into space forming the bright CME core following the cavity. Plasma that is lacking sufficient kinetic energy to escape from the Sun's gravity, falls back onto the solar surface. STEREO/NASA. The movie is available in the online supplement

and wide compared to solar cycle 23. Close to the Sun, the CME expansion is driven by the increased magnetic pressure inside the flux rope, while further out they most probably expand due to the decrease of the solar-wind dynamic pressure over distance (Lugaz et al. 2020). Therefore, the increased width for CMEs of cycle 24 may be explained by the severe drop $(\sim 50 \%)$ in the total (magnetic and plasma) heliospheric pressure (see e.g., McComas et al. 2013; Gopalswamy et al. 2014, 2015; Dagnew et al. 2020a). Interestingly, also the maximum sunspot relative number in cycle 24 reached only $65 \%$ of that from cycle $23^{13}$. The different expansion behaviors have consequences also for Space Weather effects in terms of their abilities in driving shocks (see e.g., Lugaz et al. 2017a).

\subsection{CME early evolution}

Besides the traditional observations in white-light images, also EUV or SXR imagery reveal CME signatures, presumably due to compression and heating that makes it visible in filtergrams sensible for high temperatures (see e.g., Glesener et al. 2013). Satellite missions that carry EUV instruments having large field of views can be effectively used with combined white-light coronagraph data to track CME structures for deriving the kinematical profile over their early evolution covering the CME main acceleration phase. The SECCHI instrument suite (Howard et al. 2008) aboard STEREO provides EUV and white-light data that seamlessly overlap $^{14}$ as shown in Fig. 9. For such studies one needs to keep in mind that the

\footnotetext{
13 http://sidc.be/silso/cyclesminmax.

14 SoHO EIT and C1 also provided that possibility but C1 was lost in June 1998 due to spacecraft failure. For a couple of events the usage of combined EIT-C1 data could be shown (see Gopalswamy and Thompson 2000; Zhang et al. 2001; Cliver et al. 2004b).
} 
observational data image different physical quantities (density and temperature in EUV, and density in white-light), hence, dark and bright features in both image data do not necessarily match.

From combined high temporal resolution EUV and white-light data a more detailed understanding about the energy budget (see also Sect. 8) and relation between flares, filaments and CMEs is revealed providing relevant information for SEP acceleration and generation of radio type II bursts. It is found that the thermal flare emission observed in SXR and the CME speed profile show similar behavior in timing (Zhang et al. 2001, 2004; Chen and Krall 2003; Maričić et al. 2007). For strong eruptive events an almost synchronized behavior between flare HXR emission and CME acceleration is obtained through a feedback relation (Temmer et al. 2008, 2010). The CME acceleration is found to peak already as low as about 0.5 solar radii above the solar surface (for statistics see Bein et al. 2011). Figure 10 gives the schematic profiles and distances over time between non-thermal (HXR) and thermal (SXR) flare energy release and CME kinematics (acceleration, speed).
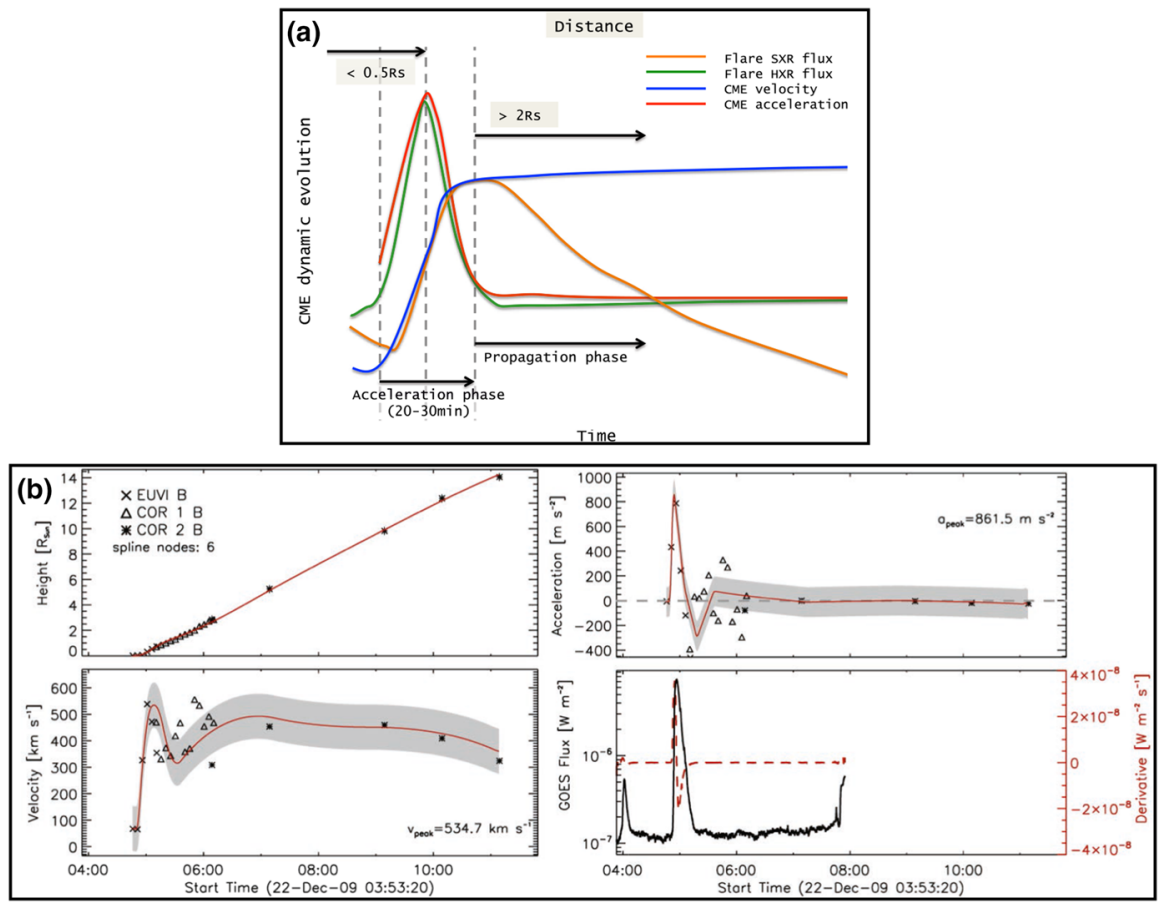

Fig. 10 CME-flare relation. a Schematics of the thermal (SXR) and non-thermal (HXR) flare energy release in comparison to the CME kinematical evolution close to the Sun. It is found that CME acceleration and HXR emission as well as the CME speed and SXR emission, respectively, are closely related. b Observational results for the December 22, 2009 CME event revealing the early evolution from combined EUV and coronagraph data (STEREO-B spacecraft) and GOES SXR flux profile for the related flare and derivative (proxy for HXR emission). Images reproduced with permission a from Temmer (2016), copyright by Wiley-VCH; and b from Bein et al. (2012), copyright by AAS 
The flare-CME feedback loop can be well explained by the CSHKP standard model (Carmichael 1964; Sturrock 1966; Hirayama 1974; Kopp and Pneuman 1976) through the magnetic reconnection process underlying both activity phenomena. In a simplistic scenario, we may summarize that magnetic reconnection drives particle acceleration (neglecting details on the actual acceleration process) leading to flare emission and closes magnetic field increasing the magnetic pressure inside the presumable CME flux rope (neglecting details on the actual magnetic configuration of the active region and surrounding). For strong flares that are related to CMEs of high acceleration values, the available free magnetic energy might be larger. This occurs preferably for CMEs initiated at lower heights where the magnetic field is stronger. With that, particles get accelerated to larger energies, hence, producing stronger flares, and more poloidal flux can be added per unit time, hence, generating a stronger expansion of the flux rope and a faster CME eruption. This is supported by theoretical investigations on the feedback process, covering magnetic reconnection with the ambient coronal magnetic field (reconnective instability, see Welsch 2018). More details are found in, e.g., Chen and Krall (2003), Vršnak et al. (2007), Vršnak (2008), Jang et al. (2017).

Associated to the erupting CME, we frequently observe coronal dimming regions that evolve over a few tens of minutes (Hudson et al. 1996; Webb et al. 2000). Core dimming regions are assumed to be located at the anchoring footpoints of the associated magnetic flux rope and reveal the loss of plasma from the corona into the CME structure adding mass to the CME body (see Temmer et al. 2017b). Secondary dimming regions most probably refer to mass depletion in the wake of the largescale magnetic field opening as the CME fully erupts (for more details on core and secondary dimming regions, see Mandrini et al. 2007). Recent studies discovered a strong relation between dimming intensity and flare reconnected flux as well CME speed (e.g., Dissauer et al. 2018, 2019). Also the final width of the CME can be estimated from the amount of magnetic flux covered by the CME associated posteruptive flare arcade as the surrounding magnetic field prevents the CME flux rope from further expansion (Moore et al. 2007). On the contrary, the CME surrounding shock as well as associated coronal waves on the solar surface, that are ignited by the lateral CME expansion, are freely propagating and are not limited in their spatial extend (for more details on globally propagating coronal waves, I refer to the Living Review by Warmuth 2015).

To derive in more detail the temporal linking of flare-CME-SEP events, image data covering large field of views for observing the lower and middle corona is of utmost importance. Figure 11 shows the different field of views of currently available and future EUV instruments to observe and study the middle corona (distance up to about 4 solar radii). The Extreme EUV Imager suite aboard Solar Orbiter works at the $174 \AA$ and $304 \AA$ EUV passbands (EUI: Rochus et al. 2020). The EUVI-LGR instrument aboard ESA's Lagrange L5 mission (launch planned for 2027) has an extended field of view to the West limb of the Sun, that is perfectly suited to track the early evolution of Earth directed CMEs from L5 view (60 degrees separation with Earth). We must not forget the capabilities of ground-based 


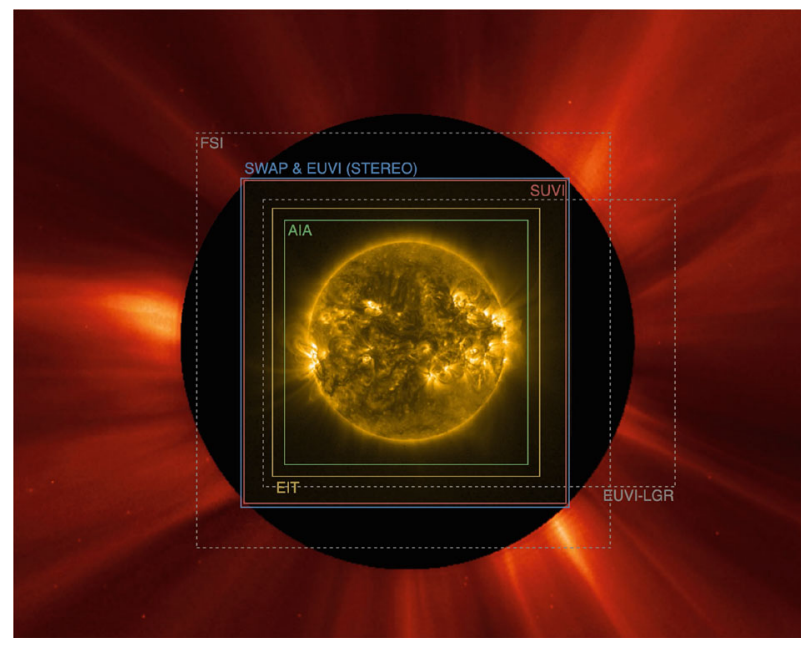

Fig. 11 EUV image from Proba-2/SWAP combined with a LASCO/C2 coronagraph image covering in total a field of view up to $\sim 4$ solar radii. The colored boxes mark the relative nominal field of views of different EUV observing instruments. FSI (Full Sun Imager is part of the EUI suite aboard Solar Orbiter), EUVI-LGR (aboard the planned L5 Lagrange mission), and SoHO/EIT. STEREO/EUVI, Proba-2/SWAP, GOES/SUVI are instruments with the largest field of view of about 1.7 solar radii. Image reproduced from http://middlecorona.com/instruments.html

coronagraph instruments such as the COSMO K-Cor at the Mauna Loa Solar Observatory in Hawaii (replaced in 2013 the aging MLSO Mk4 K-coronameter ${ }^{15}$ ) observing a field of view starting as low as 1.15 solar radii, however, quite restricted in observational time compared to satellite data.

For more details on $\mathrm{CME}$ trigger mechanisms I refer to recent review articles by Schmieder et al. (2015) or Green et al. (2018). For a more specific background on CME initiation models, see, e.g., the Living Review by Webb and Howard (2012).

\subsubsection{Shock formation, radio bursts, and relation to SEPs}

Closely related to studies of the CME early evolution and acceleration profiles, are shock formation processes. To generate a shock wave, a short-duration pulse of pressure is needed. Besides the CME, acting as piston, there is also the possibility that a strong flare energy release initiates a blast wave or simple-wave shock (e.g., Vršnak and Cliver 2008). At which height shocks are formed by an erupting disturbance is important for understanding particle acceleration processes. The acceleration profile derived from tracking the CME frontal part suggests its formation at rather low coronal heights $<1.5$ solar radii. The shock formation height itself is also strongly depending on the plasma environment. From model calculations a local minimum of the Alfvén speed is derived for a distance of about 1.2-1.8 solar radii and a local maximum around 3.8 solar radii from the Sun (Mann et al. 1999; Gopalswamy et al. 2001b; Vršnak et al. 2002). Hence, the

\footnotetext{
15 Details can be found under: https://www2.hao.ucar.edu/cosmo/documentation.
} 
statistical maximum of CME acceleration profiles is also in accordance with the local minimum of the Alfvén speed. The occurrence of such local extrema has major consequences for the formation and development of shock waves in the corona and the near-Sun interplanetary space as well as their ability to accelerate particles.

The most compelling argument for shock formation is the observation of a radio type II burst. In the case of being driven by a CME, they are reported not only to occur at the apex of a CME shock front, but also to originate from the lateral expansion of the CME as observed with LOFAR $^{16}$ (e.g., Zucca et al. 2018). Due to the large density in the lower coronal heights a large compression appears with a quasi-perpendicular geometry, favoring the shock formation process. In that respect, moving type IV radio bursts might actually represent shock signatures due to CME flank expansion, that can be used as additional diagnostics for studying the lateral evolution of a CME (Morosan et al. 2020). The SEP intensity is found to be correlated with the width of a CME, and as such identifies the CME flank region to be an efficient accelerator of particles (see Holman and Pesses 1983; Mann and Klassen 2005; Richardson et al. 2015). Comparing the CME apex and flanks, the field lines are disturbed at different heights that may lead to different onset times for the acceleration of SEPs (cf. Fig. 20). The time needed for shock formation also leads to a temporal delay of the onset of SEP events with respect to both, the initial energy release (flare) and the onset of the solar type II radio burst (evidence of shock formation). Hence, the timing is an important factor and has to be taken into account when relating these phenomena to each other.

Shocks may also be formed at larger distances from the Sun (several tens of solar radii), depending on the acceleration phase duration, the maximum expansion velocity and the width of the CME (Žic et al. 2008). Due to the declining magnetic field with distance (well defined band-splits in type II bursts can be used to estimate the magnetic field in the corona; see e.g., Vršnak et al. 2002), shocks forming at larger heights are related to softer SEP spectra (e.g., Gopalswamy et al. 2017a). As can be seen, CME acceleration, shock formation height and hardness of SEP spectra is closely connected. Compared to SEPs, which strongly depend on the magnetic connectivity with the observer, type II bursts can be observed without connectivity issues and thus, give additional information about particle acceleration processes driven by CME shocks. In that respect, type II radio bursts may be used for predicting SEPs as well as shock arrival times (e.g., Gopalswamy et al. 2008b; Cremades et al. 2015). In combination, these parameters have strong implications for Space Weather impact, revealing the importance of monitoring and studying the early evolution phase of solar eruptive events. More details on SEPs are given in Sect. 7.

Figure 12 presents a case study about the evolution of a CME front close to the Sun by using high cadence EUV images from SDO for June 12 and 13, 2010. The derived kinematics of the CME reveals a fast acceleration of its frontal part with about $1 \mathrm{~km} / \mathrm{s}^{2}$ over the distance range 1.1-2.0 solar radii. The almost vertical traces in the radio spectra are type III bursts, identified with streams of electrons (radio

\footnotetext{
${ }^{16}$ Low Frequency Array (van Haarlem et al. 2013). Recent attempts to use LOFAR for Space Weather purposes are reported under http://lofar4sw.eu.
} 

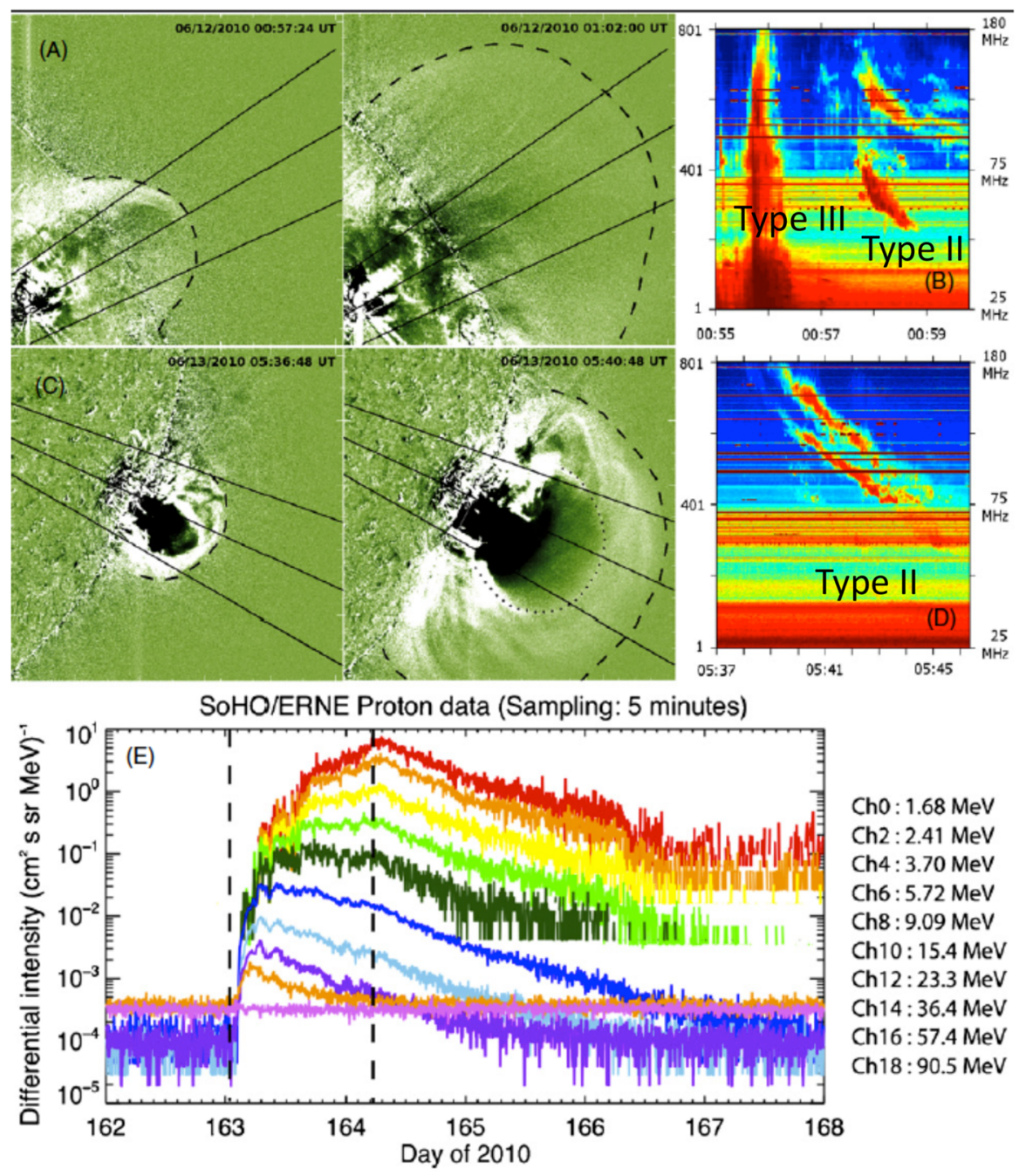

Fig. 12 Two CME events and associated coronal waves (June 12 and 13, 2010) are investigated with high cadence SDO EUV $211 \AA$ Aata. Manually tracked positions of the wavefronts are marked by dashed black lines. The connection between coronal surface wave and CME front is nicely observed. The right panels give radio data (Learmonth Observatory, Australia) revealing type III and type II bursts (upper and lower frequency band) and measurements from particle detectors at L1 (bottom panel; vertical dashed lines show AIA waves onsets). Image adapted from Kozarev et al. (2011), copyright by AAS

emission due to particles moving along open magnetic field lines), followed by diagonal structures of a moving type II burst, identified with shock waves. The onset of the type II burst appears together with the CME shock front, as observed in EUV, with a bit of a delay with respect to the shock formation that occurs close to the maximum CME acceleration. By the time the CME occurs in the LASCO field of view, the CME speed decreased to the sub-Alfvénic regime. The event produced an 
enhanced proton flux at $1 \mathrm{AU}$. However, the complex magnetic topology related to the active region prevents from making strong conclusions about the possible sites of particle acceleration (see also Kozarev et al. 2011; Ma et al. 2011; Gopalswamy et al. 2012). Definitely, more such detailed case studies combined with improved modeling of the magnetic environment is needed for advancing our understanding in the processes that accelerate particles.

\subsubsection{Stealth CMEs}

In contrast to fast and massive CMEs and their related cascade of solar surface signatures, there exist so-called stealth $C M E$ events that are most probably caused by some simple (low-energetic) magnetic field reconfiguration in the upper corona releasing magnetic flux ropes of low density that usually do not exceed the solar wind flow speed. Actually, they were recognized already in the mid 1980s and were identified as spontaneous CMEs or unassociated CMEs (meaning no surface signatures) by Wagner and Wagner (1984). Later studies showed, that they start at very large heights in the corona without noticeable signatures, such as flare emission, filament eruptions, coronal waves, or coronal dimmings (Robbrecht et al. 2009). Stealth CMEs are potential candidates to cause problem storms and missed Space Weather events, as they are hardly recognized in white-light data and due to the lack of observational imprints on the solar disk. In the recent years several studies have been published on this issue discussing those events (see e.g., D'Huys et al. 2014; Nitta and Mulligan 2017; Vourlidas and Webb 2018).

\subsection{Advantages due to multi-viewpoint observations}

In contrast to a flare, which is a rather localized phenomenon, the analyzes of CMEs and related coronal waves, propagating over large areas of the solar surface, as well as SEPs profit enormously from at least two viewpoints. The twin-spacecraft STEREO unprecedentedly provides, since its launch end of 2006, image data in EUV and white-light from multiple perspectives. STEREO consists of two identical spacecraft, STEREO Ahead (A) and Behind (B; lost signal October 2014), orbiting the Sun in a distance close to Earth, with STEREO-A being closer and STEREO-B further away from the Sun. The separation angle between the two spacecraft increases by about 45 degrees per year. ${ }^{17}$ There are four instrument packages mounted on each of the two STEREO spacecraft, SECCHI comprising EUV and white-light coronagraphs and imagers (Howard et al. 2008), IMPACT sampling the 3D distribution of solar wind plasma and magnetic field (Luhmann et al. 2008; Acuña et al. 2008), SWAVES tracking interplanetary radio bursts (Bougeret et al. 2008), and PLASTIC measuring properties of the solar wind plasma characteristics (Galvin et al. 2008). Conjoined with instruments from Earth perspective, such as SoHO (1995-), Hinode (2006-) and SDO (2010-), as well as ground based observatories (covering the radio and visual wavelength range, e.g., chromospheric

\footnotetext{
${ }^{17}$ Current position of STEREO and other spacecraft can be found under https://stereo-ssc.nascom.nasa. gov/where.shtml.
} 

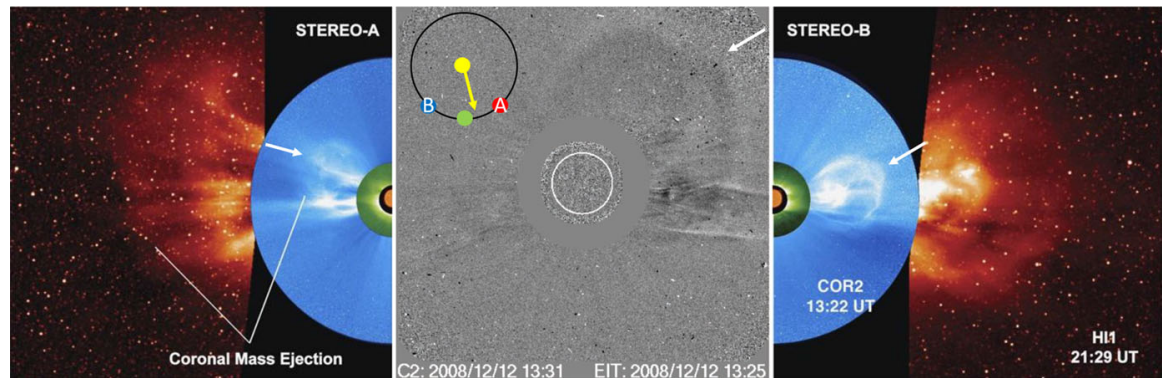

Fig. 13 Earth-directed CME from December 12, 2008 as observed from multiple perspectives. STEREOA (left) and STEREO-B (right) are separated from Earth by an angle of about 45 degrees. The running difference image from LASCO/C2 (middle panel) observes the CME as weak partial halo event. The inlay in the middle panel gives the spacecraft location (STEREO-A red filled circle; STEREO-B blue filled circle) with respect to Earth (green filled circle) and the CME propagation direction (yellow arrow). White arrows in each panel point to roughly similar parts of the CME observed with the different instruments. The closer the CME propagates in the plane-of-sky of the instrument, the higher the intensity in white-light. Image adapted from Byrne et al. (2010), copyright by Macmillan

$\mathrm{H} \alpha$ and $\mathrm{Ca}$ II lines), the evolution of active regions together with flares, CMEs, and SEPs could be for the first time stereoscopically observed. Unfortunately, a big drawback for multi-viewpoint magnetic field investigations was the lack of magnetographs onboard STEREO (this might be overcome by the ESA Lagrange L5 mission planned to be launched in 2027; see also e.g., Gopalswamy et al. 2011; Lavraud et al. 2016).

Besides having more than one vantage point, STEREO carries the heliospheric (HI) instruments, enabling to seamlessly observe the entire Sun-Earth line in whitelight. They provide a unique long-term, synoptic data-set to be exploited for Space Weather application. Wide-angle image data allow to undoubtedly link CMEs to their interplanetary counterparts (ICMEs) as measured in-situ and to investigate in detail the in-situ signatures caused by the different CME structures and orientations. More details on ICMEs are given in Sect. 6.

Figure 13 shows an Earth-directed CME observed from multiple perspectives and over a large distance range using STEREO data. From Earth perspective (shown in the middle panel), the CME is observed as weak halo event which makes it almost impossible to reliably determine a propagation direction and its radial speed. From STEREO perspective, the CME is observed close to the plane of sky of the instruments, lowering the projection effects for deriving its radial kinematics. Hence, the multiple viewpoints and homogeneous dataset of STEREO, enable to do $3 \mathrm{D}$ reconstructions of solar structures and to investigate projection effects with the attempt of correcting them, or at least limit and assess the uncertainties of the projected measurements. For SEPs, the identical instruments aboard the two spacecraft bring the advantage of having the same energy threshold, allowing systematic studies of SEPs coming from the same active region but related to a different magnetic connectivity and to probe the longitudinal dependencies.

Multi-spacecraft views enable to study especially the CME geometry and its substructures in more detail. With that, the different manifestations of shock and 

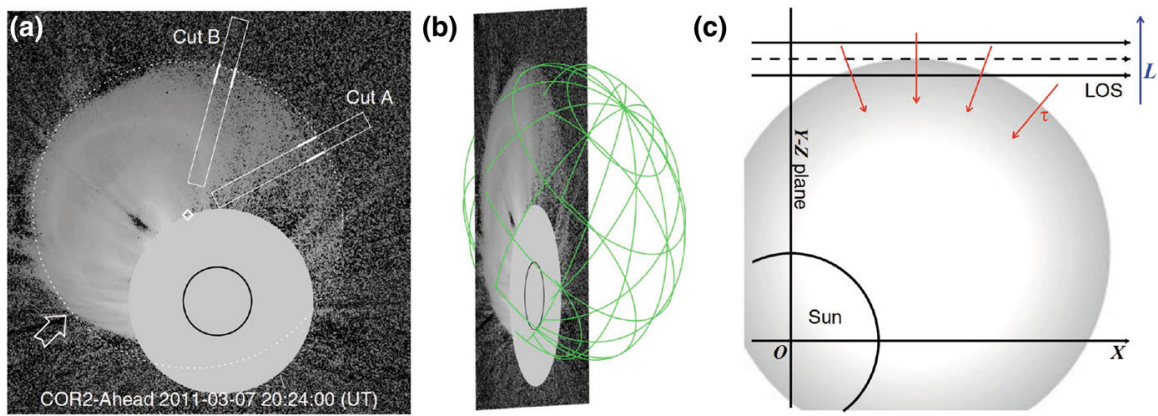

Fig. 14 CME from March 7, 2011: a Excess brightness image from STEREO-A COR2. 3D shock front (green mesh in panel (b)) projected on the image plane is shown with the dotted line. The diamond marks the geometric center of the ellipsoid model projected onto the same plane. b Excess brightness in panel (a) with the 3D shock front (green mesh) modeled with the ellipsoid model described in Kwon and Vourlidas (2017). c Geometric relation among the Sun, shell-like sheath, and line-of-sight. A partial circle around the origin $\mathrm{O}$ is the solar disk. A shell-like sheath is represented in gray color. Arrows in black, blue and red are the line-of-sight, the projected shock normal on the image plane, and the actual shock normal in 3D, respectively. Image reproduced with permission from Kwon and Vourlidas (2018), copyright by the authors

driver could be well confirmed and it is now well acknowledged that the outer envelope of the observed CME presents the expanding shock or compressed shell that encompasses the driver (e.g., Ciaravella et al. 2006; Ontiveros and Vourlidas 2009; Vourlidas et al. 2013). As the different parts have a different impact on Earth, for forecasting purposes, measurements of the CME's outer front should be clearly specified (e.g., shock front versus magnetic structure). In addition, the long-standing question whether halo CMEs would be different compared to limb events (see e.g., the Living Reviews by Chen 2011) could be solved. The shock shell of the CME can be presented as sphere-like structure expanding over 360 degrees (see Fig. 14). It was found that especially strong events (having a large compression) can be observed as halo CME independent of the viewpoint (Kwon et al. 2015; Kwon and Vourlidas 2018). In that respect STEREO data also showed that the outermost shock component of the CME matches well the solar surface structure of coronal EUV waves (Kienreich et al. 2009; Patsourakos and Vourlidas 2009; Veronig et al. 2010; Kwon and Vourlidas 2017). Therefore, observations of the surface signatures of CME related coronal waves give supportive information about the CME expansion and propagation direction and should be closely monitored for early Space Weather forecasting.

The CME speed is actually a mixture of lateral and radial expansion dynamics making it tricky to derive the "true" propagation behavior. Multiple viewpoints enable to separately study projected versus deprojected speeds and radial versus lateral expansion behaviors of CMEs. Comparing single and multiple spacecraft data revealed that single viewpoint measurements are definitely valid. However, especially measurements of the CME width (or lateral expansion) and speed for slow CMEs (deprojected speeds below $900 \mathrm{~km} / \mathrm{s}$ ) reveal high uncertainties depending on the perspective (Shen et al. 2013; Balmaceda et al. 2018). Models taking into account projection effects showed to significantly decrease the 
uncertainties in forecasting the arrival times of CMEs (e.g., Colaninno et al. 2013; Mishra and Srivastava 2013; Shi et al. 2015; Mäkelä et al. 2016; Rollett et al. 2016). A well known empirical relation exists between the radial and the lateral expansion speed, $V_{\text {rad }}=0.88 V_{\text {exp }}$, as described by Dal Lago et al. (2003) and Schwenn et al. (2005). Follow-on studies showed that this relation can also be described by the CME half-width, $w$ (assuming a cone model), given by $f(w)=$ $1 / 2(1+\cot w)$ and that the kinematics of extremely fast CMEs is better estimated by $V_{\text {rad }} \approx V_{\exp }$ (Michalek et al. 2009). Moreover, statistical studies revealed that the relationship between the radial and lateral expansion speed is a linear function, hinting towards the self-similar expansion behavior of CMEs already close to the Sun (Vourlidas et al. 2017; Balmaceda et al. 2020). However, in the low corona, for some events a strong overexpansion is observed (e.g., Patsourakos et al. 2010). The assumption of a rather self similar expansion is found to be valid for most of CME events when propagating in interplanetary space (e.g., Bothmer and Schwenn 1998; Leitner et al. 2007; Démoulin 2008; Gulisano et al. 2012; Vršnak et al. 2019).

Since we cannot gather the full complexity of the CME structure, idealized geometries assuming self-similar expansion, act as basis of many CME models and $3 \mathrm{D}$ reconstruction techniques that were developed over the past years. Basic models make use of a simple cone-type geometry (e.g., St Cyr et al. 2000; Schwenn et al. 2005; Michalek et al. 2006; Xie et al. 2004). With the availability of image data from multiple views, those tools were refined and full 3D reconstructions were enabled from which estimates of the deprojected kinematics, geometry, and propagation direction are derived. Methods comprise, e.g., inter-image tie points and triangulation in various wavelength ranges (see e.g., Harrison et al. 2008; Howard and Tappin 2008; Maloney et al. 2009; Reiner et al. 2009; Temmer et al. 2009; Liewer et al. 2010), forward models related to white-light data (e.g., Wood et al. 2009), or center of mass calculations (Colaninno and Vourlidas 2009). Also online tools were made available, such as e.g., the CCMC tools StereoCat. ${ }^{18}$ A well known and widely used technique is the graduated cylindrical shell (GCS) forward model developed by Thernisien et al. (2006, 2009). Coronagraph image data showing the CME from at least two different vantage points are required, on that an idealized flux rope structure in the form of a croissant is fitted. The GCS model depends on a number of free parameters, such as the flux-rope height and angular width as well as the aspect ratio which determines the rate of self-similar expansion. Figure 15 gives the 3D reconstruction of two CME events using GCS applied on STEREO and LASCO data in a study by Patsourakos et al. (2016). Especially for multiple events, the investigation and determination of the cause of geoeffectiveness is rather challenging as the processes happening on the Sun are complex. In that respect, geometrical fitting methods help to derive the propagation direction of a particular solar event in order to reliably link it to a geoeffective event at Earth. In a similar way, stereoscopy can be applied also on radio data. Figure 16 shows results from so-called goniopolarimetric observations using WIND and STEREO spacecraft data studying the location of radio type II bursts. That method is used to derive

\footnotetext{
18 StereoCat https://ccmc.gsfc.nasa.gov/analysis/stereo/.
} 


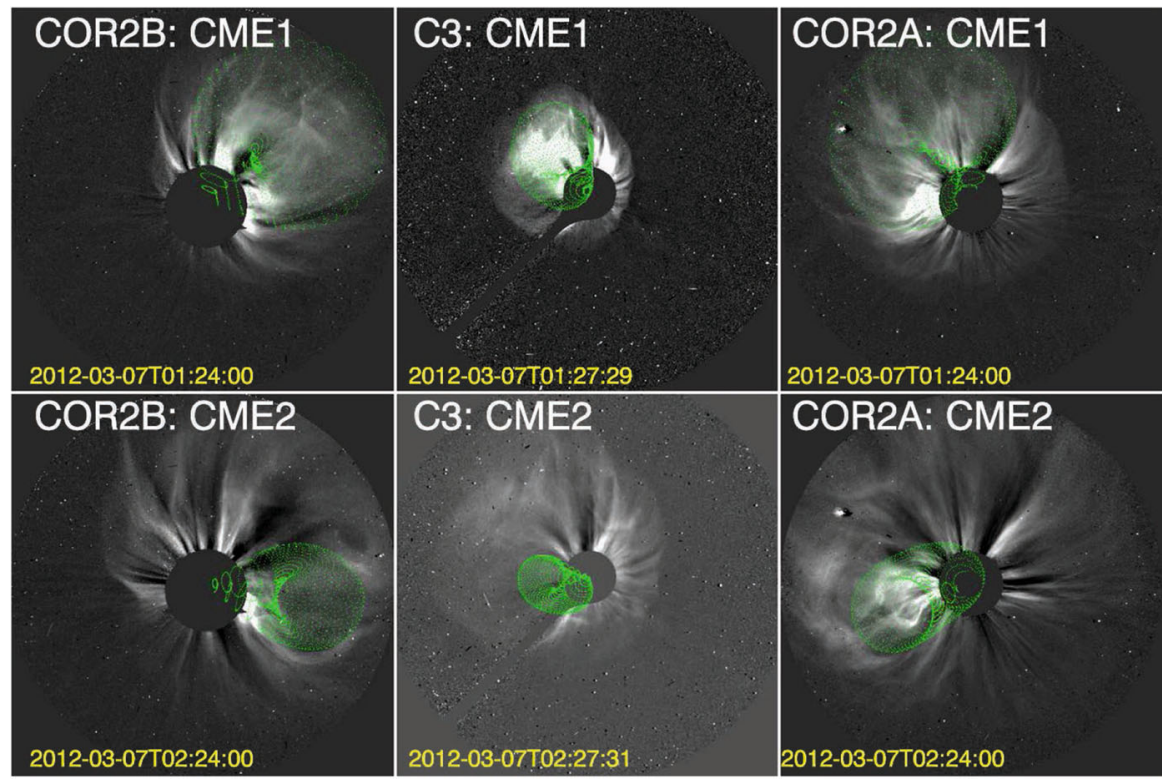

Fig. 15 March 7-11, 2012: GCS fitting (green mesh) of two CMEs (CME1: top panels. CME2: bottom panels-note that CME1 is visible as extended bright structure in these images) using white-light data from the three spacecraft, STEREO-A, SOHO and STEREO-B. The first, second, and third columns contain coronagraph images from COR2 aboard STEREO-B, C3 aboard SOHO, and COR2 aboard STEREO-A, respectively. Image reproduced with permission from Patsourakos et al. (2016), copyright by AAS
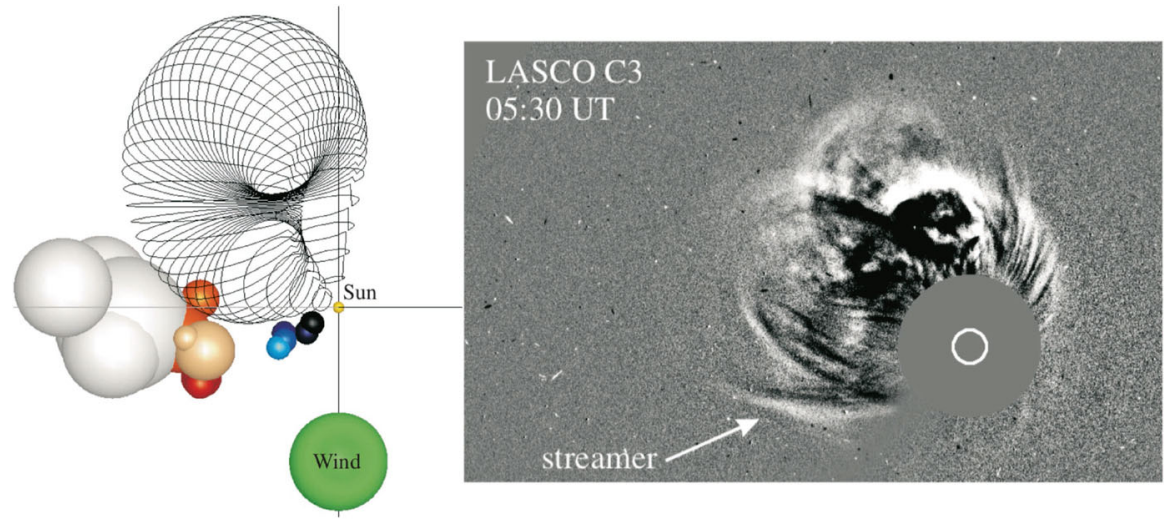

Fig. 16 Left panel: View of the flux rope and radio sources as seen from Earth. The CME flux rope obtained from GCS 3D reconstruction (black grid croissant) together with the 3D reconstruction of the radio type II burst (dark and light blue spheres) using gonopolarimetric technique. Right panel: SOHO/ LASCO C3 difference image showing the CME as seen from Earth, for comparison with the left panel. Image adapted from Magdalenić et al. (2014), copyright by AGU 
the direction of arrival of an incoming electromagnetic radio wave, its flux, and its polarization.

Besides geometry related information, STEREO coronagraph data can also be used to derive the CME deprojected mass close to the Sun (Colaninno and Vourlidas 2009; Bein et al. 2013) which, together with the early acceleration phase, are taken for better estimating the energy budget between flares and CMEs (see also Sect. 8). STEREO and its wide-angle HI instruments also enable to derive the 3D geometry of compressed density structures like CIRs (see Rouillard et al. 2008; Wood et al. 2010). Sometimes the disentanglement between CMEs and CIRs in HI is tricky, hence, it needs careful inspection of the data when tracking specific features (see e.g., Davis et al. 2010).

Using multi-viewpoint data and applying different reconstruction techniques we vastly gained important insight about CME characteristics. Moreover, the results stemming from STEREO observations clearly challenged existing CME and SEP models. However, using idealized geometries, the real 3D structure of a CME or SEP paths can only be approximated and we need to keep in mind that there are strong deviations from these. Especially, in interplanetary space, the geometry of the CME front clearly changes, as flanks and nose interact differently with the nonuniform solar wind (pancaking effect; see e.g., Riley and Crooker 2004; NievesChinchilla et al. 2012). In addition, the CME shape might vary due to intensity changes as the relative position to the Thomson sphere changes, that makes the tracking of specific white light structures complicated (e.g., Vourlidas and Howard 2006). Therefore, the derived deprojected values and forecasts based on them still need to be treated with caution (see also Mierla et al. 2010; Riley et al. 2018). For more details on the complex interactions of CMEs with their surroundings, I refer to the review by Manchester et al. (2017).

For more information on solar stereoscopy and tomography techniques, applied to various large-scale structures in the solar corona, I refer to the Living Review by Aschwanden (2011). For comprehensive investigations, the EU funded project HELCATS $^{19}$ established databases ready to use for analyzing STEREO 3D CME characteristics and HI CME tracks on a statistical basis (see e.g., Murray et al. 2018; Barnes et al. 2019). Out of that an extensive ICME catalogue ${ }^{20}$ was compiled by Möstl et al. (2017) and Palmerio et al. (2018). A conjunction catalogue covering CME in-situ measurements by two or more radially aligned spacecraft (MESSENGER, Venus Express, STEREO, Wind/ACE) is given by Salman et al. (2020).

\section{Interplanetary counterparts of CMEs: ICMEs}

Newly developed imaging capabilities clearly enhanced our understanding about the relation between solar eruptions, CMEs, and their counterparts in interplanetary space (ICMEs). SMEI, the Solar Mass Ejection Imager (SMEI: Eyles et al. 2003) on the Earth-orbiting Coriolis spacecraft, was the first heliospheric white-light imaging

\footnotetext{
${ }^{19}$ Heliospheric Cataloguing, Analysis and Techniques Service; https://www.helcats-fp7.eu.

20 https://helioforecast.space/icmecat.
} 
instrument covering the Sun-Earth space (for more details see the review by Howard and Harrison 2013). The successor of SMEI are the heliospheric imagers (HI: Eyles et al. 2009) aboard STEREO (Kaiser et al. 2008). The WISPR instrument (Vourlidas et al. 2019) aboard the Parker Solar Probe mission and the SoloHI instrument (Howard et al. 2020) aboard Solar Orbiter build upon the STEREO/HI heritage and make similar observations of the inner heliosphere. The observational principle is like a coronagraph, but as these are wide-angle instruments, they observe much larger distances from the Sun. These unprecedented image data facilitated the tracking of CMEs through interplanetary space and with that could unambiguously relate the CME white-light structure to in-situ measurements (see e.g., Möstl et al. 2009, 2014) and moreover, get better insight on how CMEs interact with the ambient solar wind structures. Figure 17 shows a so-called Jmap which is constructed from running difference white-light $\mathrm{HI}$ data covering the Sun-Earth distance range. By extracting the central part of the HI images in the horizontal direction, the ICME front can be rather easily followed as function of the elongation angle. Before further analysis, the elongation-time measurements need to be converted into radial distance. For that, methods assume either a certain CME geometry and apply the propagation direction of the CME (see e.g., Lugaz 2010) or use fitting functions (see e.g., Rouillard et al. 2008). These procedures cover rather high uncertainties in the derived kinematical profiles, that needs to be taken into account when interpreting CME propagation profiles for interplanetary space (e.g., Rollett et al. 2012; Liu et al. 2013).

It is well known that CMEs during their propagation phase tend to get adjusted to the ambient solar wind flow owing to the drag force exerted by the ambient solar wind (Gopalswamy et al. 2001a; Wang et al. 2004). As consequence, CMEs which are faster than the ambient flow speed get decelerated while those which are slower get accelerated. This alters their speed, hence, travel time and with that has impact on Space Weather forecasting. The adjustment to the ambient flow speed happens

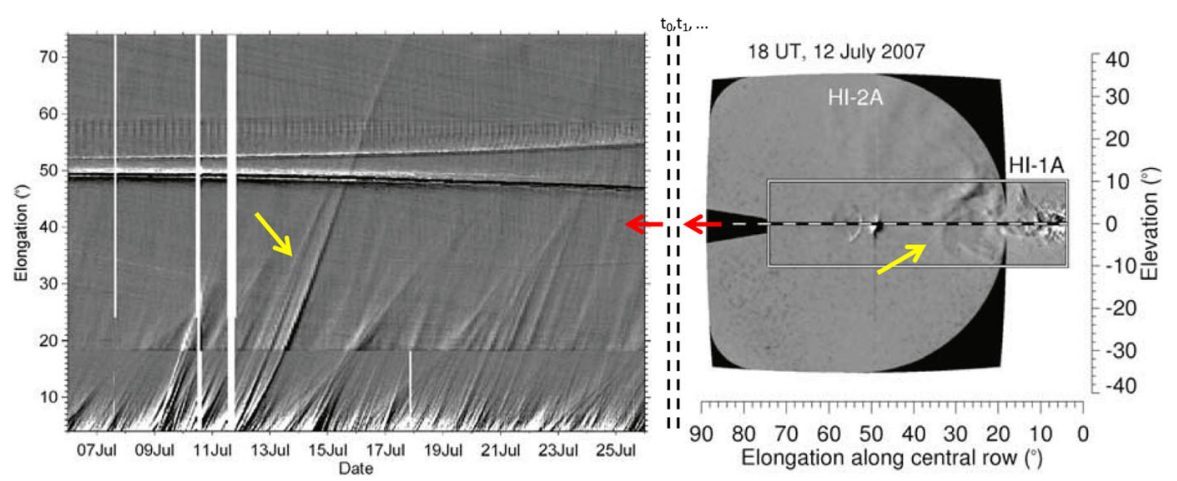

Fig. 17 From running difference STEREO-A HI1+2 image data the central rows are extracted (right) at each time step and rotated by 90 degrees (middle). From this a time-elongation plot (so-called Jmap) is constructed (left). The CME front is marked by a yellow arrow in the direct image as well as in the Jmap. Image adapted from Davies et al. (2009), copyright by AGU 
most probably in interplanetary space (e.g., Sachdeva et al. 2015). At which distance exactly depends on the competing forces acting on a CME, Lorentz versus drag force (e.g., Vršnak 2008). The longer the CME is driven, hence, the longer the magnetic reconnection process is ongoing (which might be inferred from flare emission and growing post eruptive arcades), the farther away from the Sun the adjustment may occur. Empirical relations found between CME kinematics and flare properties (flare ribbons, coronal dimmings, or post-eruptive arcade regions) actually may be used to estimate the reconnected flux that empowers the CME (e.g., Gopalswamy et al. 2017b; Temmer et al. 2017b; Dissauer et al. 2018; Tschernitz et al. 2018). ${ }^{21}$ The amount of drag from the solar wind depends on the relative speed and density between the solar wind and the CME as well as the CME width/size. It is found that wide CMEs of low mass tend to adjust rather quickly to the solar wind speed and, hence, their transit time (i.e., how long a CME needs to traverse a certain distance) is determined primarily by the flow speed in interplanetary space. Narrow and massive CMEs propagating in a fast solar wind have the shortest transit times (see e.g., Vršnak et al. 2010).

Figure 18 shows typical in-situ signatures of a well-defined ICME at $1 \mathrm{AU}$, revealing a simultaneous jump of all measured components (shock) with a subsequent sheath structure (compressed plasma) of increased density, speed and turbulence, that is followed by signatures of a smooth and enhanced magnetic field together with a rotation as observed in the vector components (changing from plus to minus or vice versa). The ICME magnetic structure is usually identified by that smooth field rotation (flux rope), a plasma-beta lower than 1 (referring to a dominant magnetic component), a low temperature, and a linearly decreasing proton speed (see also the Living Review by Kilpua et al. 2017). Sometimes that flux rope can be associated with twisted structures observed already in white-light image data. Having a long-lasting southward directed magnetic field (measured in the $B_{\mathrm{z}}$ component), flux ropes are the main contribution of strong geoeffectiveness. The passage of rather isolated magnetic ejecta at 1 AU typically takes about 1 day (cf. Richardson and Cane 2010). ${ }^{22}$ Hence, geomagnetic disturbances may last for many hours. Flank hits, interacting CMEs and complex ejecta, can have passage durations of about 3 days at 1 AU (see Burlaga et al. 2002; Xie et al. 2006; Marubashi and Lepping 2007; Möstl et al. 2010), affecting the Earth's atmospheric layers over a much longer time range, and, hence, causing stronger geomagnetic effects (see also Sect. 9.4).

By combining remote sensing and in-situ data using multi-spacecraft reconstruction methods, it is revealed that from in-situ measurements we observe localized variations of the magnetic field behavior that may not be representative of the global structure (see Möstl et al. 2008, 2009; Rouillard et al. 2010; Farrugia et al. 2011; DeForest et al. 2013). Studies using multi-spacecraft encounters separated in radial distance and longitude give insight on the magnetic coherence of

\footnotetext{
21 A database of more than 3000 solar flare ribbon events observed by SDO and reconnection flux is given in Kazachenko et al. (2017).

22 A near-Earth ICME catalogue is given under: http://www.srl.caltech.edu/ACE/ASC/DATA/level3/ icmetable2.htm.
} 


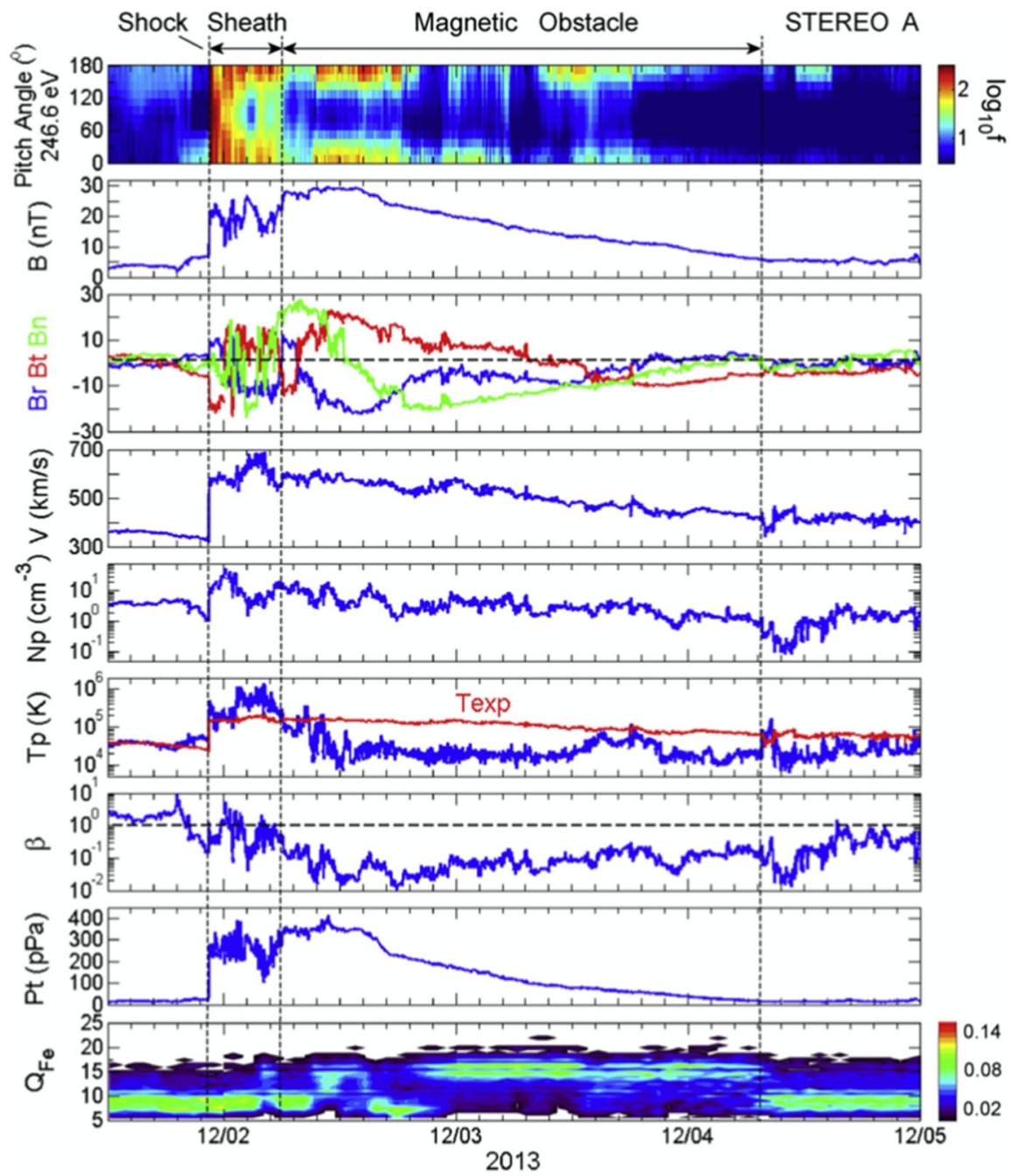

Fig. 18 STEREO-A in-situ measurements and identification of a CME together with its closed magnetic structure. From top to bottom: pitch-angle distribution data of suprathermal electrons, total magnetic field intensity, magnetic field vectors (in RTN coordinates), solar wind proton bulk speed, proton number density, proton temperature (in red the expected proton temperature is given calculated from an empirical relation to the solar wind speed as given by Richardson and Cane 1995), plasma-beta, total pressure, distribution of the iron charge state. Vertical dashed lines mark the shock-sheath, and the boundaries of the magnetic structure. Image reproduced with permission from Jian et al. (2018), copyright by AAS

ICMEs on various scales and with that raise questions on the inner structure of CMEs as well as their interaction processes with the interplanetary magnetic field (see e.g., Good et al. 2018; Lugaz et al. 2018). Using flux rope reconstruction methods applied on in-situ measurements (see e.g., Al-Haddad et al. 2013) a comparison between the physical parameters derived close at the Sun with those 
measured in-situ can be performed enabling to interpret changes in the mass, flux, etc. due to the interaction with the interplanetary solar wind (see e.g., Bisi et al. 2010; Temmer et al. 2017b, 2021). Especially the reconnection of the magnetic flux rope with the interplanetary magnetic field is found to lead to either a loss of magnetic flux (so-called erosion) or adding of magnetic flux (see e.g., Dasso et al. 2007; Manchester et al. 2014; Ruffenach et al. 2015). Removing or adding magnetic flux may lead to a change in the ICME propagation behavior. Filament material is found less often from in-situ measurements (identified by low charge state species) despite the fact that most CMEs are accompanied by filament eruptions. Heating mechanisms or simply missing the cold filament material due to the localized in-situ measurements might be a reason for that (Filippov and Koutchmy 2002). This is supported by findings that magnetic ejecta are only partly filled with hot plasma related to heating by the flare (e.g., Gopalswamy et al. 2013a).

More details on the relation between white light remote sensing image data and in-situ measurements, including proper nomenclature, is given by Rouillard (2011). A review on multi-point ICME encounters before and during the early years of STEREO is given by Kilpua et al. (2011). The recent review by Luhmann et al. (2020) comprises a thorough overview on the ICME propagation in the inner heliosphere.

\section{Solar energetic particles (SEPs)}

\subsection{General characteristics}

SEP events are observed in-situ as enhanced electron, proton and heavy ion flux (and as increased level of cosmic rays on ground) largely exceeding the thermal energy levels, ranging from $\mathrm{keV}$ to $\mathrm{GeV}$. Strong fluxes of energetic protons (socalled proton events) cause strongest geoeffective phenomena. High energy SEPs in the range of $\mathrm{GeV}$ reach the Earth within less than 10 minutes and may produce ground level enhancements (GLE; measurable in neutron monitors at Earth surface), that are of special interest as they have major effects on crewed spaceflight and aircraft due to the increased radiation exposure (e.g., Malandraki and Crosby 2018).

In general, there are two populations of SEP events, gradual and impulsive ones (e.g., Reames 1999, cf. Fig. 19). It is the different temporal scaling which is disentangling those two populations. Driving agents acting on longer time scales are related to CME shock acceleration mechanisms. However, gradual events seem to be accompanied as well by an impulsive part which is thought to be related to shorttime magnetic reconnection processes, as observed in flares. Obviously gradual events are caused by both driving agents prolonging the acceleration process but on a less energetic level (see also Anastasiadis et al. 2019). Impulsive events are also obtained to be related to those SEP events where the location of the particle accelerator is magnetically well connected to the observer. SEP/GLE events are found to have the hardest spectra and the largest initial acceleration (Gopalswamy et al. 2016). There are still many open issues about the processes leading to 
(a)

Gradual SEP events
(CME shocks in corona

and IP space)
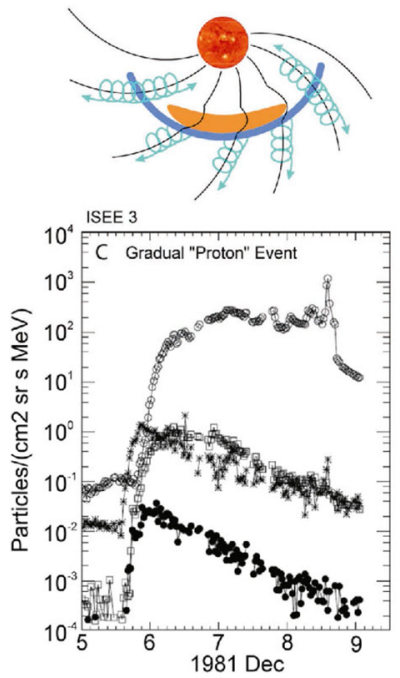

(b) Impulsive SEP events (acceleration in
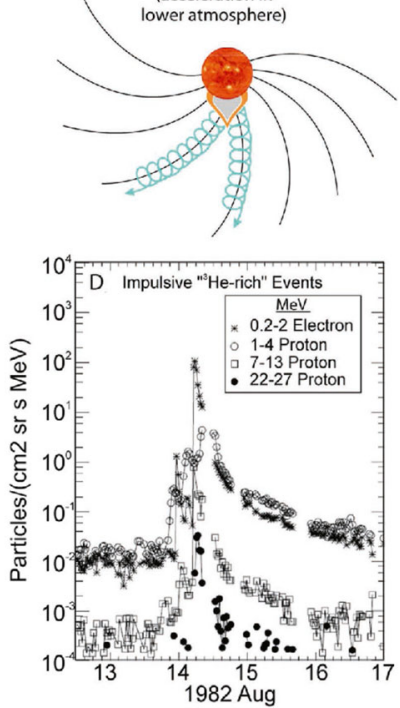

Fig. 19 Separation into gradual and impulsive SEP events and their suggested driving mechanisms. a Gradual SEP events result from diffusive acceleration of particles by large-scale shocks produced by CMEs and populate interplanetary space over a wide range of longitudes. b Impulsive SEP events result from acceleration during magnetic reconnection processes in solar flares and are observed well when magnetically connected to the flare site. Intensity-time profiles of electrons and protons in $\mathbf{c}$ gradual and d impulsive SEP events. Image reproduced with permission from Desai and Giacalone (2016) after Reames (1999)

energetic particles as well as about their (suprathermal) seed populations in the corona and interplanetary space (e.g., Mason et al. 1999; Desai et al. 2006; Mewaldt et al. 2012). Clearly, the primary condition for the production of SEPs is the opening of magnetic field lines into interplanetary space (as for eruptive events) and that accelerated particles have access to that open field lines. It is confirmed that for confined flares no SEPs are observed (e.g., Trottet et al. 2015). As shocks play an important role in the acceleration of particles, coronal shock waves on the solar surface and interplanetary space related to CMEs as well as interacting CMEs are investigated in relation to SEPs (see e.g., Park et al. 2013; Lario and Karelitz 2014; Miteva et al. 2014, and related MHD modeling results, e.g., Pomoell et al. 2008).

SEP events that become Space Weather effective are controlled by many factors, such as the source region location of the eruption (longitude and latitude) and width of the CME, background solar wind, seed populations, multiple CMEs and their interaction, or magnetic field configuration near the shock. For example, narrow CMEs (slow or fast) do not efficiently accelerate particles (see e.g., Kahler et al. 2019), and CMEs originating from the eastern hemisphere are less likely to create a SEP event near the Earth because of the weak magnetic connection between Sun and the Earth. The highest-energy particles are most likely accelerated close to the 
shock nose where the shock is strongest, while the lower energy particles are accelerated at all regions (see e.g., Bemporad and Mancuso 2011; Gopalswamy et al. 2018a). Hence, also the ecliptic distance to the shock nose, i.e., the event source region latitude, is an important parameter for SEP prediction (see e.g., Gopalswamy et al. 2013b).

Knowledge about the magnetic connectivity is a crucial parameter in order to detect SEPs in-situ and to relate them to the proper driving agent (Reames 2009). Figure 20 depicts the propagating idealized circular-shaped CME shock front in relation to the radially oriented magnetic field lines near the Sun. The cartoon describes a scenario in that a narrow range of heights (2-4 solar radii) exists where compression is sufficient for effective particle acceleration (e.g., Cliver et al. 2004a) and for having a good connection to the observer, with heights increasing at the eastern and western flanks. Concluding, the connectivity changes with distance from the Sun. On the other hand, flare locations lying close to open structures like coronal holes, have different magnetic configuration and facilitate the acceleration of particles into the heliosphere (Cane et al. 1988; Reames et al. 1996; Shen et al. 2006). In that respect, the interplay between open and closed magnetic field is important to know and due to the lack of observations needs to be supported by reliable coronal modeling.

For Space Weather forecasting purposes, it is desired to derive clear signatures showing that particles associated to eruptive events were able to escape to the high corona and interplanetary space. Therefore, the monitoring of possible radio emission (from decimetre and longer waves) is found to be of utmost importance (e.g., Klein et al. 2010). Observations of flares in the high energy range provide additional information on the location, energy spectra, and composition of the flare accelerated energetic particles at the Sun that can be compared to 1AU SEP events

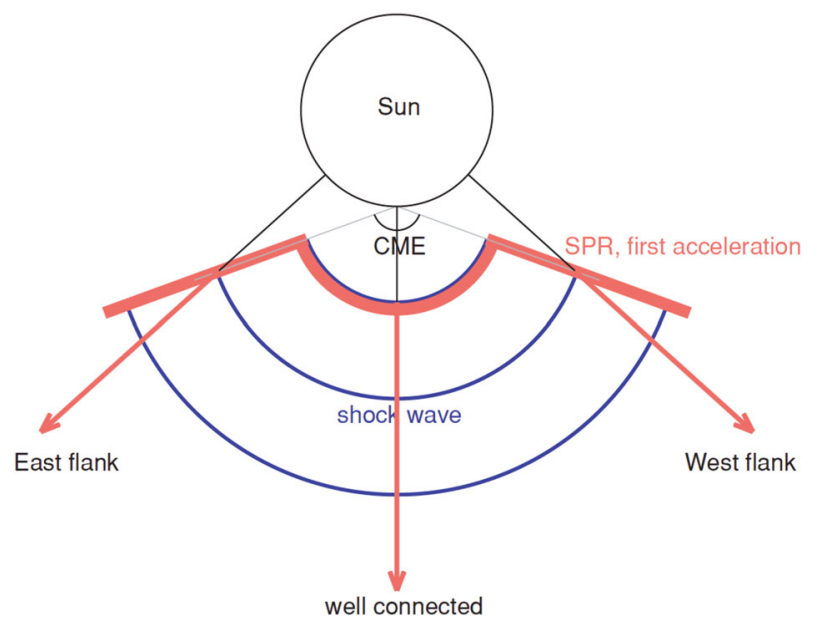

Fig. 20 Cartoon showing a possible acceleration scenario for SEPs. The radial field lines (black lines) are hit by the CME shock front (blue) at different heights for the nose and the flank. The solar particle release (SPR) likely begins at a 2-4 solar radii (marked by the red region) for the apex or higher up for the flanks. Image reproduced with permission from Reames (2009), copyright by AAS 
(Lin 2006). RHESSI imaging capabilities could show that flare $\gamma$-ray sources are not co-spatial with flare HXR sources (Fletcher et al. 2011). Laurenza et al. (2009) developed a technique for short-term forecasting of SEPs based on flare coordinates and flare flux together with the time-integrated intensity of SXRs and type III radio emission $(\sim 1 \mathrm{MHz})$. Similar, the forecast of the occurrence of SEP events could be determined using the peak ratios in flare fluxes measured over $(0.05-0.4 \mathrm{~nm}) /$ (0.1-0.8 nm) as described by Kahler and Ling (2018). For improved SEP forecasting, it is suggested to take into account parameters from both driving agents, flares and CMEs (see Klein and Dalla 2017). Figure 21 shows for an eruptive event the timing between flare SXR emission (GOES flux), radio type III burst (WIND), electron and proton spectra measured at $1 \mathrm{AU}$ (SOHO), and combined white-light image data from the ground-based K-Cor coronagraph and LASCO/C2 instrument. Especially, observations of the early evolution of CMEs and derivation of shock formation heights (see also Sect. 5.2.1) as well as distribution of Mach numbers along the shock surface (Rouillard et al. 2016) might give some lead time for SEP

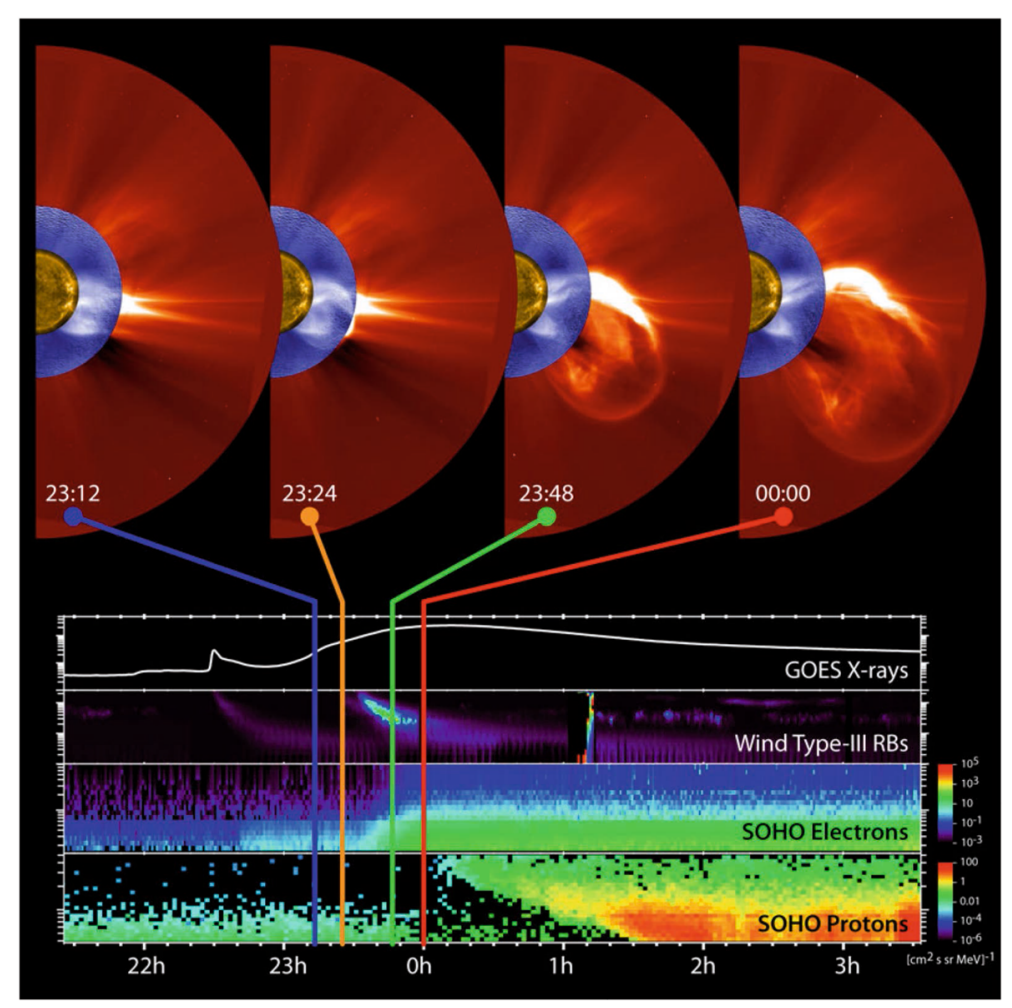

Fig. 21 In the top row, the January 1, 2016, eruptive event appears in the sequence of images from SDO AIA (gold), MLSO K-Cor (blue), and SOHO/LASCO (red). A fast CME associated with an SEP event detected near Earth, is seen appearing off the southwest limb of the Sun. The time profiles reveal that data from the ground-based K-Cor coronagraph could be used for a timely warning of particle events as described in that case study. Image reproduced with permission from the issue cover of St Cyr et al. (2017), copyright by AGU 
forecasting. Monitoring the generation of flare associated coronal surface waves (EIT/EUV waves; see Thompson et al. 1998), gives additional hints on shocks ignited by the CME lateral expansion (for confined events no coronal waves are observed).

\subsection{SEPs observed from multiple viewpoints}

Using STEREO data, the observation of wide-spread SEP events shed new light on the possible generation mechanisms together with the lateral expansion of CMEs and their interaction with other coronal structures (see Rouillard et al. 2012). Figure 22 gives for the February 25, 2014 SEP event intensity profiles as measured by different spacecraft that are separated from Earth by 152 degrees (STEREO-A) and 160 degrees (STEREO-B). The SEP producing eruptive event is located at E82 (marked by the green arrow). As STEREO-B is closest to the SEP source, that flux profile reveals the highest intensity. The related CME was observed as halo event from Earth, having a projected speed of more than $2000 \mathrm{~km} / \mathrm{s}$ and before that, other CMEs were launched from that region. SEPs may be directed to wide-spread angles by field line draping around the closed magnetic field of the CME and/or complex magnetic fields due to CME-CME conglomerates, or CME-CIR interaction (e.g., Dresing et al. 2016, 2018; Gómez-Herrero et al. 2011, 2017; Xie et al. 2017; Guo et al. 2018). For more details on wide-spread SEP events, including a comprehensive catalogue see Paassilta et al. (2018).

During solar cycle 24 strong SEP events produced only two GLEs, that could be related to CMEs launched from the Sun on May 17, 2012 and September 10, 2017. Gopalswamy et al. (2013b) did a comparative study between the GLE May 17, 2012

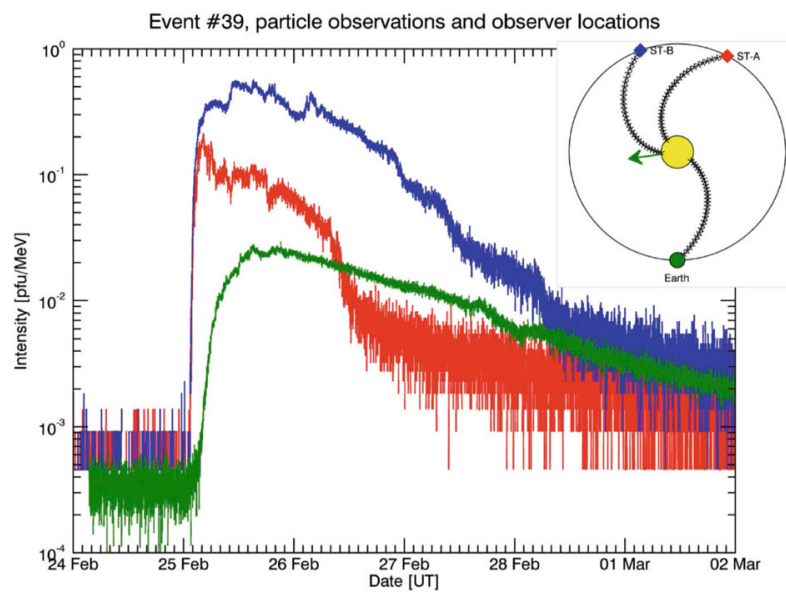

Fig. 22 February 25, 2014 SEP event and detected proton intensities (red = STEREO-A/HET, blue = STEREO-B/HET, green $=$ SOHO/ERNE). Inset: relative locations of the STEREO spacecraft and the Earth during the event. The arrow pointing out from the Sun shows the location of the SEP producing active region (at longitude E82), and the asterisks mark the nominal Parker spiral magnetic field lines connecting each observer to the Sun. Image reproduced with permission from Paassilta et al. (2018), copyright by Springer 


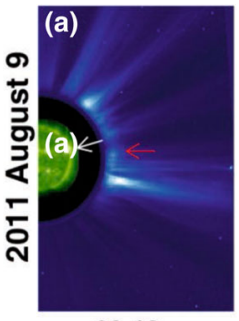

08:12

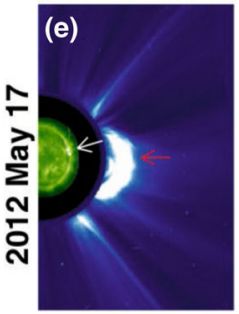

01:48

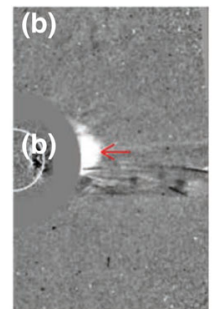

08:12

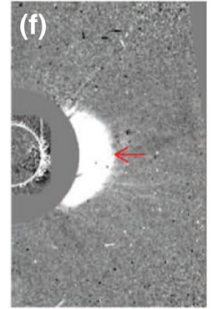

01:48

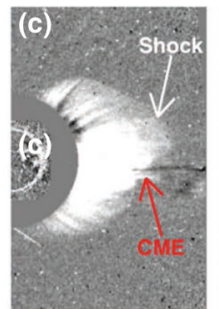

08:24

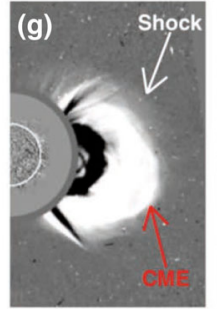

02:00

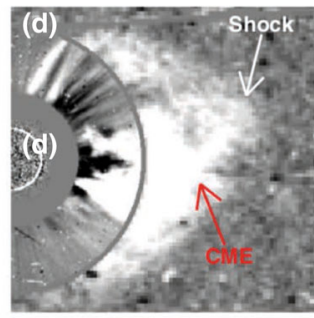

08:42

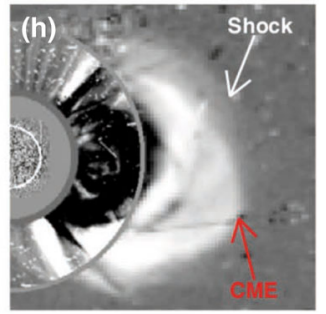

02:18

Fig. 23 Coronagraph images from SOHO/LASCO (left panel: direct image; other panels to the right: difference images) showing the evolution of two CMEs from August 9, 2011 and from May 17, 2012 (GLE event). Inlay images are from SDO/AIA $193 \AA$ showing the solar sources. Red arrows point to the CME nose. The May 17, 2012 event remains bright over the LASCO field of view and its shock structure is located close to the CME $(\mathbf{g}, \mathbf{h})$. The August 9, 2011 event reveals a smaller CME main body and a wider shock structure $(\mathbf{c}, \mathbf{d})$, which is a sign of a weak shock. Image reproduced with permission from Gopalswamy et al. (2013b), copyright by AAS

and a non-GLE CME event from August 9, 2011 using STEREO image data as given in Fig. 23. The study revealed for the GLE event a shock formation height very low in the corona (1.38 solar radii from solar center) and that the shock remained closer to the driver structure over the coronagraphic field of view. This is indicative of a stronger shock that is driven over a longer time and, hence, can produce very energetic particles. Rouillard et al. (2016) modelled for the May 17, 2012 event the background topology of the magnetic field using multiple viewpoints to derive the geometry of the shock front and to find where particles get accelerated most efficiently (see also Plotnikov et al. 2017; Kouloumvakos et al. 2019). Particles that get magnetically trapped in between CME structures pose a particle reservoir that may play a key role in the late acceleration of gradual SEPs related to CME-CME interaction events (e.g., Lugaz et al. 2017b). Despite these tremendous enhancements in our knowledge gained from stereoscopic observational data, still a major drawback in unraveling the SEP nature is the unknown configuration of the interplanetary magnetic field along which SEPs propagate and internal distribution (Kahler and Vourlidas 2013). Therefore, the acceleration process of SEPs seems not to be spatially limited but happens over a wide range of longitudes including transport before being injected at distant longitudes (e.g., Vlahos et al. 2019; Kozarev et al. 2017; Malandraki and Crosby 2018).

For recent reviews on SEP events covering in detail the production and acceleration processes, I refer to the Living Review by Desai and Giacalone (2016) 
or the book by Reames (2017). The review by Lugaz et al. (2017b) is focusing on SEPs with respect to CME-CME interaction events and the Living Reviews by Kilpua et al. (2017) on particle acceleration due to ICME shocks.

\section{Energy budget between flares, CMEs, and SEPs}

Figure 24 schematically shows the relevant components of energy build-up and dissipation processes. How much of the free energy is actually released and to which parts that released energy partitions into primary and secondary processes can only be answered by statistics. In cutting-edge studies performed by Emslie et al. (2004, 2012), a sample of eruptive events was investigated with respect to the energy release and its distribution into different components, concluding that about a third of the total available energy might be released in an eruptive event. Similar conclusions are drawn by Gopalswamy (2018) from calculating for extreme eruptive events the ratio of the total reconnected flux to the available active region flux. However, discrepancies were found from simulation studies (e.g., Reeves et al. 2010). Recent extensive statistics by Aschwanden et al. (2017), yield that $\sim 87 \%$ of the magnetic energy is released. About $10 \%$ of the released energy drives the CME and $\sim 80 \%$ goes into particle acceleration. In total about $10 \%$ percent of the free magnetic energy goes particularly into SEP acceleration. With respect to the CME, SEPs dissipate about 3\% of the CME kinetic energy (similar as derived by Emslie

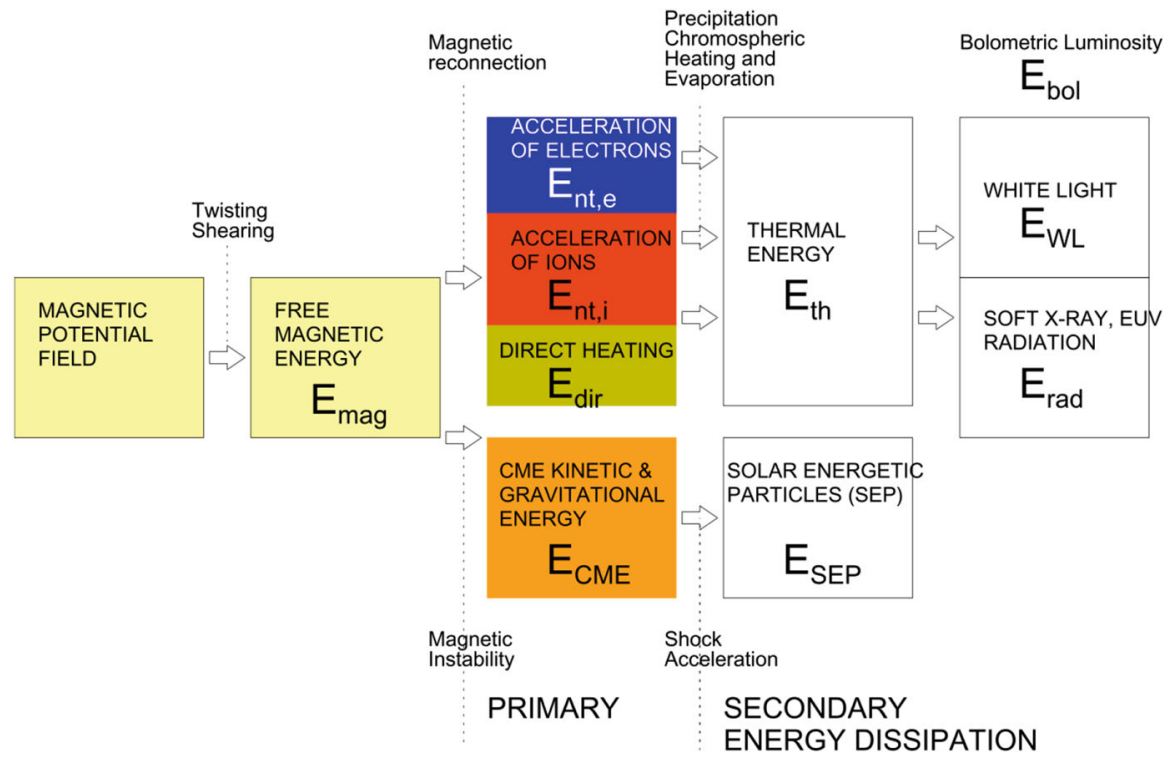

Fig. 24 Schematic diagram showing the different energy dissipation processes of the observed activity phenomena. The chart covers the energy input (light yellow shaded boxes) and energy dissipation via primary (colored boxes) and secondary processes (white boxes). Those are the major processes identified for studying their energy closure relationship. Image reproduced with permission from Aschwanden et al. (2017), copyright by AAS 
et al. 2012). The CME velocity shows strongest correlations with SEP characteristics and all that is consistent with CME-driven shock acceleration (see Mewaldt 2006; Papaioannou et al. 2016).

The discrepancies in the results show that there might be processes that cannot be disentangled from each other, cover energy conversion (e.g., non-thermal into thermal due to cooling), or are simply not well observed. Nevertheless, the conclusion is that the free magnetic energy of an active region is sufficient to generate flare-CME-SEP events and with that confirms their common magnetic origin. For the interested reader I refer to the book by Aschwanden (2019).

\section{Structuring of interplanetary space: the solar wind}

The solar wind is the major hub in interplanetary space dictating how fast disturbances may evolve and guiding the motion of accelerated particles. Knowledge about the prevailing structure of the solar wind in terms of plasma and magnetic field distribution is therefore of utmost importance in order to obtain reliable Space Weather forecasts. In turn, this also leads to a better understanding and interpretation of the propagation behavior of CMEs as well as the occurrence and energetics of SEPs. Interplanetary space is strongly shaped by the interplay between slow and fast solar wind flows, causing stream interaction and compression regions, transient disturbances such as shocks and closed magnetic structures (flux ropes) of evolving CMEs. These structures pose magnetic barriers that are able to change the propagation characteristics of a specific CME and affect SEP fluxes. In the following, I will focus on solar wind structures relevant for CMEs and SEPs. For more details on the heliospheric magnetic field, I refer to the Living Review by Owens and Forsyth (2013) and for solar wind stream interaction regions throughout the heliosphere to the Living Review by Richardson (2018).

\subsection{General characteristics}

The solar wind is a continuous flow of charged particles propagating radially outward from the hot solar corona into interplanetary space. The characteristics of the solar wind are measured in-situ at specific locations such as the Lagrangian point L1 close to Earth (ACE, WIND, DSCOVR), from satellites orbiting planets (e.g., BepiColombo, VEX, MESSENGER, MAVEN), STEREO having varying longitudinal separation close to Earth's orbit, or PSP and Solar Orbiter with special mission trajectories in the inner heliosphere (cf. Fig. 2). Figure 25 shows the solar wind speed measurements from spacecraft Ulysses which had the goal to examine the poles of the Sun (Wenzel et al. 1992). In total, Ulysses performed three polar orbits, with each one taking six years to complete, over different phases of the solar cycle 22 and 23. The first one covers the solar minimum phase revealing slow solar wind streams over the equator and a fast wind over the poles where $\mathrm{CHs}$ are situated. The second orbit happened over solar maximum activity and shows the intermix of fast and slow winds at all latitudes. Three quarters of the third orbit were completed during the minimum of the next solar cycle. 


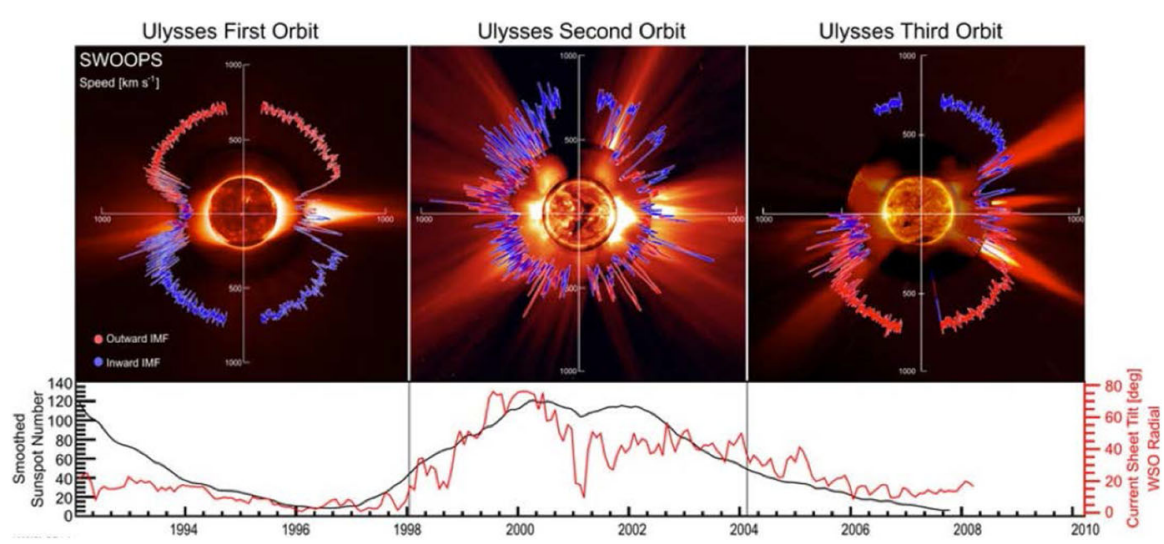

Fig. 25 Solar wind variation over the solar cycle. Outward interplanetary magnetic field in blue, inward interplanetary magnetic field in red. The bottom panel shows the timeline and line graphs of the relative smoothed sunspot number. Note also the inverted interplanetary magnetic field lines between the first and third orbit due to the reversal of the magnetic field. Courtesy: ESA

Besides the observed changes over latitude, the solar wind characteristics differ over distance. Early missions like Helios 1 and 2 (Schwenn et al. 1975; Rosenbauer et al. 1977), achieved a perihelion of 0.29 AU and gathered valuable information about the solar wind characteristics close to the Sun. Figure 26 shows the radial dependence of the solar wind parameters over the Sun-Earth distance derived from HELIOS (1974-1981) and OMNI (1963-2016) observations at 1 AU. The study by Venzmer and Bothmer (2018) extrapolates that information to regions as close as 10 solar radii, based on an empirical solar-wind model for the inner heliosphere in dependence on the solar cycle. Solar wind measurements from PSP at a distance of about 35 solar radii basically confirm the results from these earlier missions and their derived radial scalings, but also obtain that the magnetic field is very strongly fluctuating (Bale et al. 2019; Kasper et al. 2019). ${ }^{23}$

The left panel of Fig. 27 schematically depicts various large-scale structures in the interplanetary space. We differentiate between the heliospheric current sheet, separating opposite magnetic field, solar wind streams of different speeds, and closed magnetic fields of CMEs that, due to their rapid expansion, act as pistons creating shocks. The interaction between fast and slow streams (plasma volume with frozen-in magnetic field) leads to compression, forming so-called stream interaction regions (SIRs) to the West, and rarefaction regions to the East. On the large scale it is assumed that the interplanetary dynamics can be described by ideal MHD equations. The right panel of Fig. 27 gives the results from a simulation using a 3D MHD model (see more details on coronal and solar wind MHD modeling in the Living Review by Gombosi et al. 2018). The model results obtain a warping of the heliospheric current sheet and show the latitudinal dependence of the fast solar wind stream in the radial direction (Wilcox et al. 1980). All that reveals the complexity

${ }^{23}$ For recent PSP result see also special issues of the Astrophysical Journal Supplement Series and Astronomy and Astrophysics at https://iopscience.iop.org/journal/0067-0049/page/Early_Results_from_ Parker_Solar_Probe and https://www.aanda.org/component/toc/?task=topic\&id=1326. 

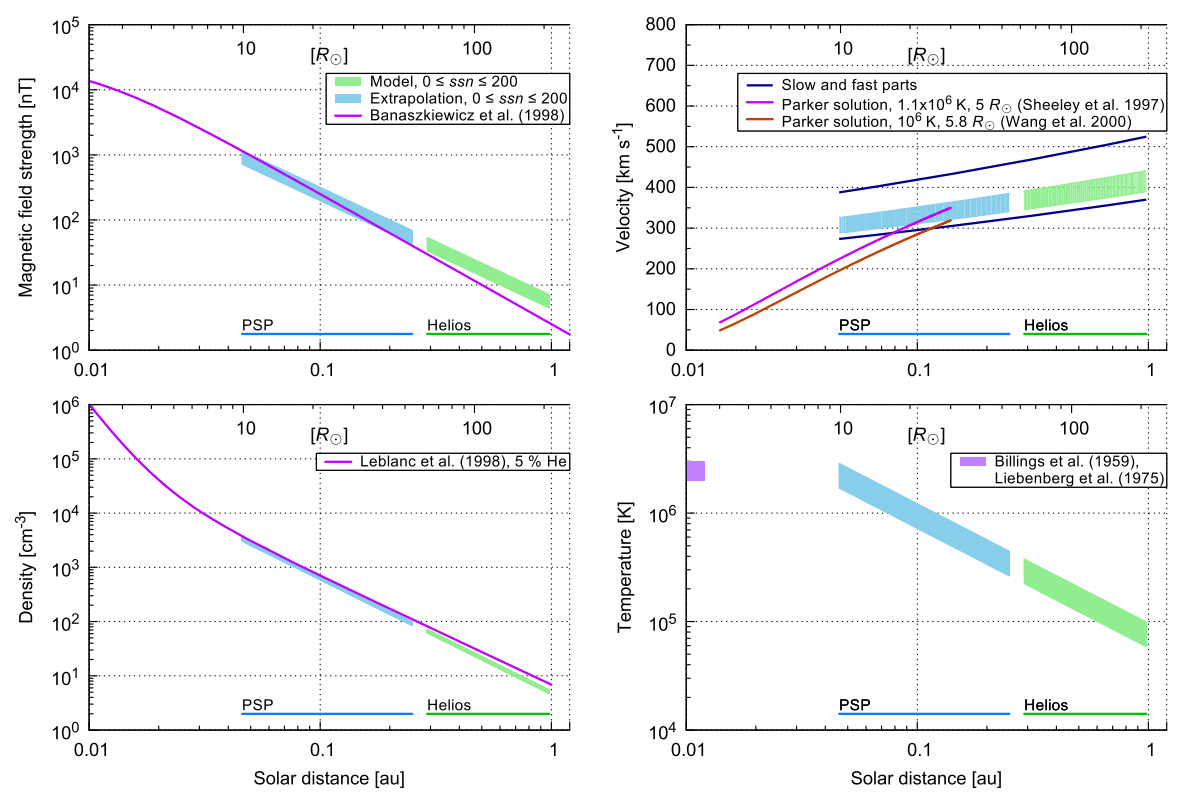

Fig. 26 Radial profiles of solar-wind parameters for the Sun-Earth distance. Median values are obtained from Helios and OMNI measurements and are extrapolated to the PSP orbit region as close as 10 solar radii. The lower edges of the shaded areas correspond to solar minimum and the upper edges to solar maximum. As comparison, overplotted are model results from Banaszkiewicz et al. (1998), from Sheeley et al. (1997) and Wang et al. (2000) for the slow solar wind speed, from Leblanc et al. (1998) for the density and the range of temperature measurements given by Billings (1959) and Liebenberg et al. (1975). Image reproduced with permission from Venzmer and Bothmer (2018), copyright by ESO
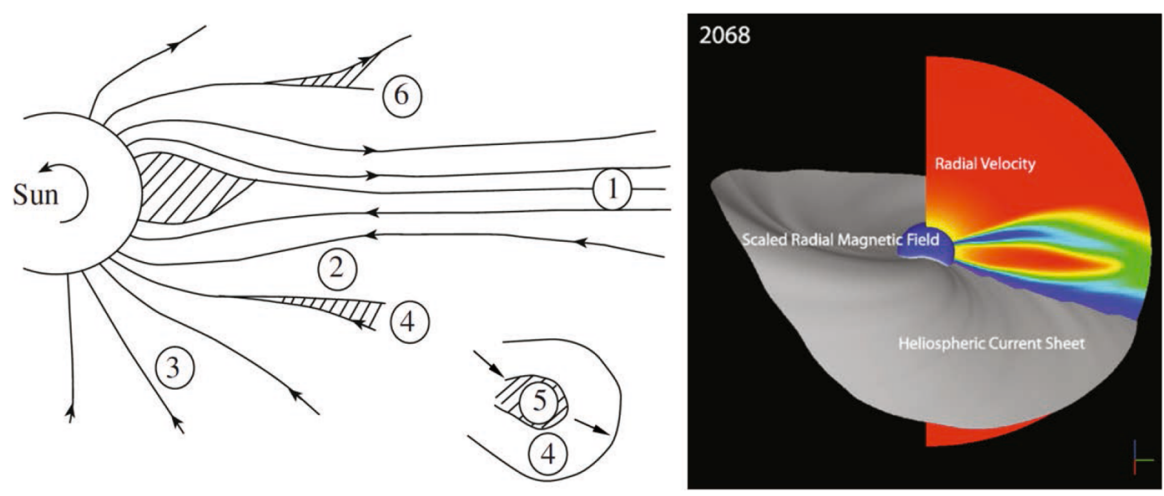

Fig. 27 Left: schematic drawing of the different large scale structures in interplanetary space. Digits designate (1) heliospheric current sheet, (2) slow streams from coronal streamers, (3) fast streams from coronal holes, (4) compressed plasma (CIR on the front of fast and slow streams, and sheath region before the leading edge of a "piston"), (5) "pistons" (such as a magnetic cloud or ejecta), (6) rarefaction region. Right: Large-scale properties of the inner heliosphere (out to $1 \mathrm{AU}$ ) for Carrington Rotation 2068 from a global MHD solution. The two meridional slices in each panel show the radial velocity and radial magnetic-field strength, scaled to $1 \mathrm{AU}$. The slice in the equatorial plane shows the scaled number density. The sphere at 30 solar radii shows the scaled radial magnetic-field strength. Images reproduced with permission from [left] Yermolaev et al. (2009), copyright by Pleiades; and from [right] Riley et al. (2011), copyright by the authors 
and interplay between open and closed magnetic field structures, occurring with different dynamics. For more details on the multi-scale nature of the solar wind, I refer to the Living Review by Verscharen et al. (2019).

The observed large-scale structures of the solar wind are intimately connected to the coronal magnetic field originating from the solar photosphere. The quasi-steady fast wind ( $>450 \mathrm{~km} / \mathrm{s}$ ) emanates from coronal holes, locations of predominantly open magnetic field, while the variable slow component is believed to originate mostly from closed magnetic field configurations around the streamer belt (McComas et al. 2000). Coronal holes are observed as low density and low temperature structures, and therefore appear as dark areas in the wavelength ranges of EUV and SXR, imaging coronal temperatures of a few million Kelvin. Figure 28 depicts the interplay between slow and fast solar wind streams. After a coronal hole passed the central part of the solar disk, in-situ measurements reveal about 1-2 days later an increase in the density and magnetic field, and about 3-4 days later in the plasma speed (Vršnak et al. 2007). Since coronal holes, and with that the fast component of the solar wind, are long-lived structures, SIRs can often be observed for several solar rotations, and are correspondingly called co-rotating interaction regions (CIRs) when observed more than once. The leading edge of a CIR represents a forward pressure wave and the trailing edge of a CIR a reverse pressure wave (cf. right panel of Fig. 28; for more details see the review by Cranmer et al. 2017). These waves may develop into shocks, and as such, large periodically recurrent coronal holes may cause geomagnetic storms roughly appearing with the frequency of the solar rotation, i.e., every 27 days (e.g., Rotter et al. 2012). During times of low solar activity, induced storms by recurrent CIRs may put equally much

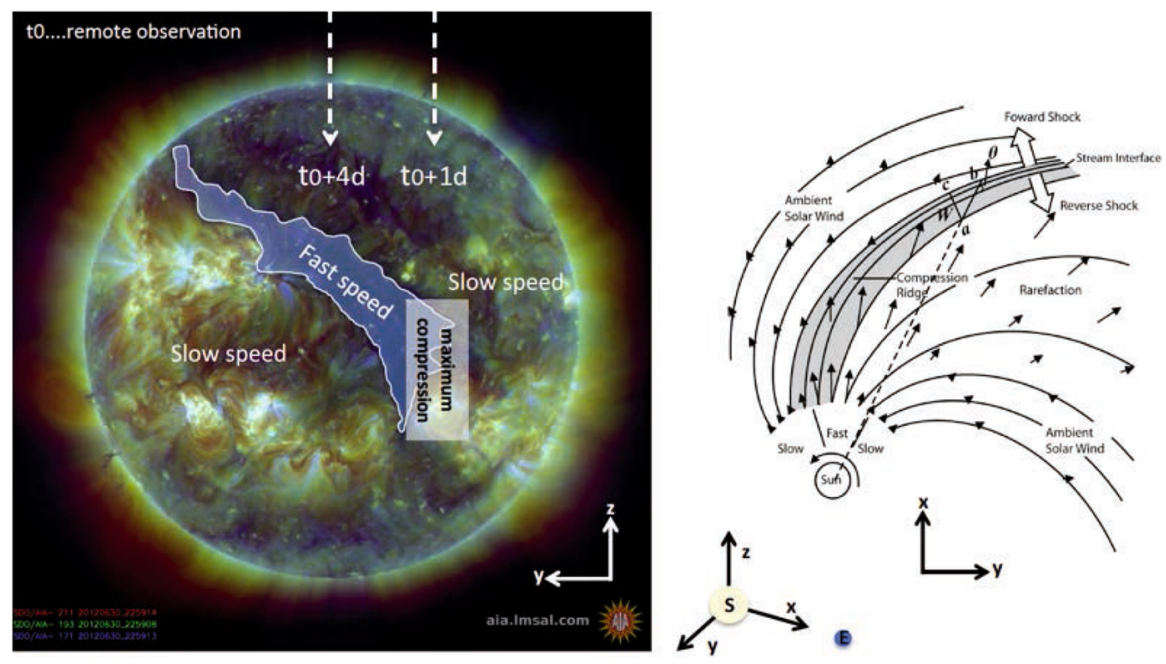

Fig. 28 Left: SDO/AIA composite image of the wavelength channels 211-193-171 $\AA$ from June 30, 2012 showing the reduced density region of a coronal hole (shaded area). At the time to, the coronal hole reaches a central position. From in-situ data at $1 \mathrm{AU}$ about 1 day later the maximum in the density/magnetic field is measured and about 4 days later the maximum in the speed/temperature. Right: Fundamental processes involved in the 3D dynamics of stream evolution, adapted from Pizzo (1978) 


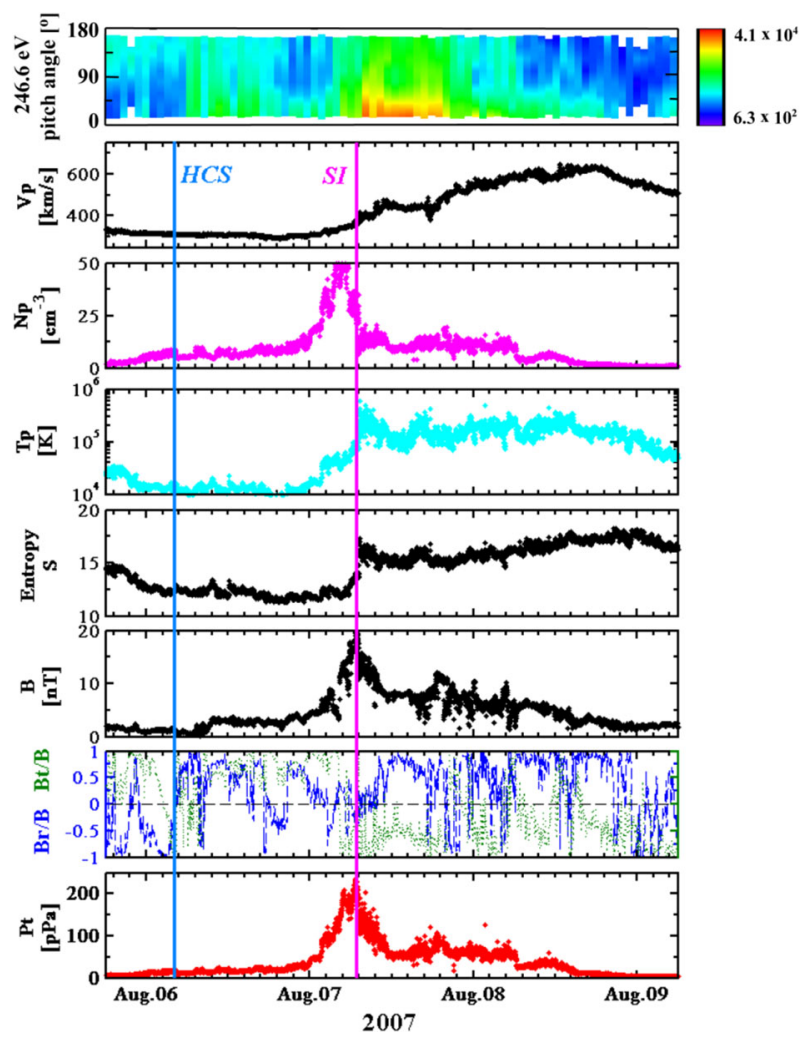

Fig. 29 STEREO-A in-situ solar wind measurements of a SIR and identification of the stream interface (SI; given by a magenta vertical line marking the peak of the total perpendicular pressure). As blue vertical line, the heliospheric current sheet (HCS) is marking the sector structure of different interplanetary magnetic field polarity. Top to bottom panels: pitch-angle distribution data of suprathermal electrons, solar wind proton bulk speed, proton number density, proton temperature, entropy, total magnetic field intensity, the ratios of the radial and transversal component of the magnetic field, total perpendicular pressure. Image reproduced with permission from Jian et al. (2009), copyright by the authors

energy into the Earth's magnetosphere-ionosphere system as CMEs (e.g., Richardson et al. 2001; Tsurutani et al. 2006). On average, the strongest geomagnetic storms due to CIRs occur during the early declining phase of a solar cycle (e.g., Verbanac et al. 2013; Grandin et al. 2019). Compared to CMEs, CIRs may drive prolonged geomagnetic activity and cause strong high energy particle enhancements in the Earth's radiation belts (e.g., Reeves et al. 2003; Miyoshi et al. 2013; Kilpua et al. 2015). As the solar wind parameters vary with the level of solar activity, so does their geoeffectiveness (see e.g., Jian et al. 2011; Richardson and Cane 2012; Watari 2018). There is substantial effort in the solar and heliospheric physics community to improve the understanding and modeling of the spatial and temporal distribution of solar wind plasma and magnetic field properties (see Cranmer and Winebarger 2019). 
Since the source regions of slow and fast streams on the Sun, namely closed and open magnetic field, are different, their intermix affects detailed analyzes of the solar wind. Therefore, it is suggested that solar wind studies should be organized by the origin of the solar wind plasma (Schwenn 1983; Zhao et al. 2009; Borovsky et al. 2019). As minimum requirement, it is accepted to distinguish between the different solar wind structures by their in-situ measured plasma (density, speed, temperature) and magnetic field characteristics. A categorization scheme developed by $\mathrm{Xu}$ and Borovsky (2015) can be applied to separate the solar wind plasma into four types, namely, coronal-hole-origin (fast solar wind), streamer-belt-origin (slow solar wind), sector-reversal-region (plasma from top of helmet streamers), and ejecta (solar transients, such as CMEs).

Figure 29 shows typical plasma and magnetic field characteristics for a well defined SIR at 1 AU. The SIR measurements reveal an abrupt drop in density, a simultaneous rise in the proton temperature, and an East-West flow deflection (see e.g., Jian et al. 2006, 2019). The stream interface (SI), given by a rather symmetric profile in the total perpendicular pressure peaking shortly after the density maximum, separates originally slow, dense plasma from originally fast, thin plasma back at the Sun (e.g., Wimmer-Schweingruber et al. 1997). The behaviour of the suprathermal electrons gives additional information about the topology, hence, connectivity of the interplanetary magnetic field lines between the Sun and the observer. That also allows to investigate, e.g., the Parker spiral versus non-Parker spiral orientation of the magnetic field and the related open flux versus closed or disconnected flux natures of the magnetic field (see Owens and Forsyth 2013). SIR signatures differ clearly from those of ICMEs (cf. Fig. 18) and as such, the specific characteristics can be applied to identify SIRs and CMEs from visual inspection of the in-situ solar wind plasma and magnetic field measurements. Together with the information of transit times (can be roughly derived from the average in-situ speed of the specific structure) we can link the in-situ signatures back onto the solar surface to study their relation. Table 1 gives average values of characteristic parameters for the different structures observed, such as slow and fast speed solar wind streams, CME sheath and magnetic ejecta. The time range used for that statistics covers the years 1976-2000 (see Yermolaev et al. 2009). As can be seen,

Table 1 Properties of the different solar wind types derived from OMNI data analyzed over the period 1976-2000

\begin{tabular}{lllll}
\hline & Fast wind & Slow wind & CMEs (shock-sheath) & CME (magnetic ejecta) \\
\hline$v_{\mathrm{p}}, \mathrm{kms}^{-1}$ & $>450-500$ & $<400-450$ & $\sim 450 \pm 110$ & $\sim 410 \pm 110$ \\
$n_{\mathrm{p}}, \mathrm{cm}^{-3}$ & $6.6 \pm 5.1$ & $10.8 \pm 7.1$ & $14.3 \pm 10.6$ & $10.1 \pm 8.0$ \\
$B, \mathrm{nT}$ & $6.4 \pm 3.5$ & $5.9 \pm 2.9$ & $8.5 \pm 4.5$ & $12.0 \pm 5.2$ \\
$T_{\mathrm{p}} \times 10^{4} \mathrm{~K}$ & $13.1 \pm 11.8$ & $4.4 \pm 4.4$ & $12.9 \pm 17.6$ & $4.5 \pm 6.6$ \\
Dst, nT & $-28.7 \pm 25.9$ & $-10.7 \pm 18.2$ & $-21.5 \pm 33.0$ & $-52.1 \pm 45.8$
\end{tabular}

Average values and standard deviations are given for the flow speed $\left(v_{\mathrm{p}}\right)$, proton density $\left(n_{\mathrm{p}}\right)$, total magnetic field $(B)$, and proton temperature $\left(T_{\mathrm{p}}\right)$. In addition, the geoeffectiveness of the different solar wind types is given as measured by the disturbance storm time (Dst). Taken from Table 3 in Yermolaev et al. (2009) 
the values reveal large standard deviations reflecting the variations of the interplanetary conditions over the solar cycle. Similar as the activity level changes over time with respect to the occurrence of sunspots, active regions, flares and CMEs (see also Sect. 5), also the occurrence of coronal holes varies having implications on the global structuring of interplanetary space (e.g., Harvey and Recely 2002; Heinemann et al. 2019).

\subsection{Background solar wind}

Open magnetic flux from the solar surface structures interplanetary space and with that influences CME and SEP propagation behavior. We assume that the majority of open flux originates within coronal holes. This is supported by an empirical relation linking the size of coronal holes observed on the solar surface to the in-situ solar wind plasma and magnetic field measured at 1 AU a few days later (Vršnak et al. 2007). In detail, the width of a coronal hole, i.e., its longitudinal extension, is found to be strongly related to the in-situ measured peak speed (Garton et al. 2018). Deriving the solar wind characteristics stemming from a specific coronal hole, is found to be tricky as each coronal hole evolves rather individually and the surrounding solar surface structures may play a major role in shaping the interplanetary solar wind (Heinemann et al. 2020). Still, an open discussion is the discrepancy between estimates of open solar magnetic flux from remote photospheric and in-situ spacecraft observations that may differ by as much as a factor of two (e.g., Arden et al. 2014; Linker et al. 2017; Wallace et al. 2019). This suggests a fundamental issue in our understanding about the topology of the coronal magnetic field and the energization of plasma, hence, the acceleration of fast solar wind flows. Recent studies find that the open magnetic field within coronal holes is predominantly concentrated in unipolar magnetic flux tubes with high outflow velocities but covering only a fraction of about $10 \%$ of the coronal hole area (see Akiyama et al. 2013; Wiegelmann et al. 2014; Hofmeister et al. 2017). For more details on modeling the coronal magnetic field and open solar flux see e.g., the Living Reviews by Mackay and Yeates (2012) and Lockwood (2013).

Due to the rather slow evolution of coronal holes, a legitimate assumption is that the solar wind parameters do not vary strongly over the duration of an entire solar rotation. Based on that, to forecast the occurrence of high speed solar wind streams at Earth, L1 spacecraft data may simply be forward shifted over one Carrington rotation period (27.28 days). These so-called persistence models are found to work remarkably well (see e.g., Owens et al. 2013; Reiss et al. 2016). However, spacecraft used for forecasting may be located over different latitudes and in-situ measurements gather the characteristics of rather localized structures within that large scale three dimensional objects. The spatial restrictions of such localized structures are demonstrated in Fig. 30, showing how solar wind streams appear differently as measured by STEREO-A and STEREO-B spacecraft that are separated in latitude by $\sim 10$ degrees. The data cover two Carrington rotations (2076 and 2077) revealing a solar wind stream well observed by STEREO-B, but clearly missed by STEREO-A. For comparison, the top panel of Fig. 30 gives the photospheric magnetic field configuration on the Sun using magnetic field 


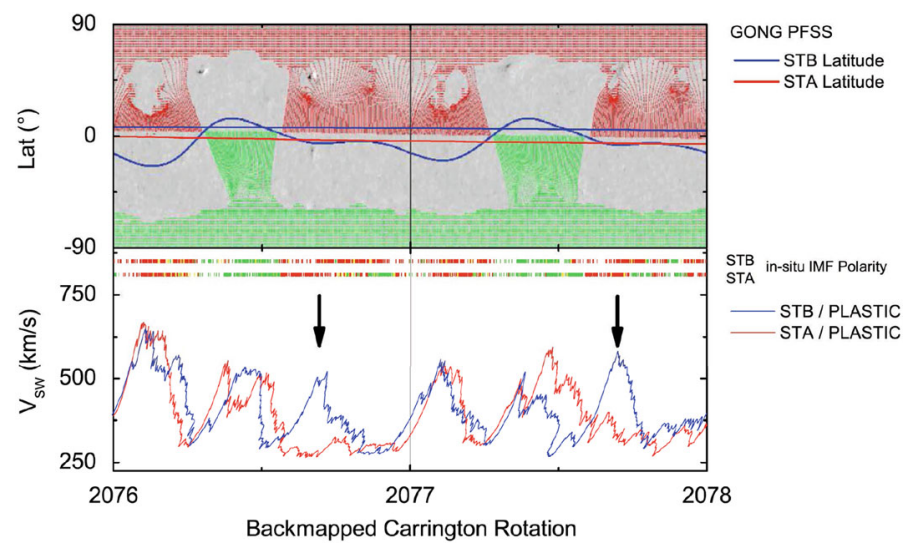

Fig. 30 Top panel: synoptic map from GONG overlaid with magnetic field extrapolation results from the PFSS (potential field solar surface; see e.g., Schrijver and De Rosa 2003) model showing the location of open field lines at the ecliptic plane. Bottom panel: solar wind bulk speed measured by PLASTIC instruments aboard STEREO-A (red) and STEREO-B (blue). Colored bands on the top part of this panel represent the interplanetary magnetic field polarity. Black arrows mark the solar wind stream observed over two rotations (CR 2076 and 2077) by STEREO-B, but missed by STEREO-A due to the latitudinal separation of the spacecraft. Image reproduced with permission from Gómez-Herrero et al. (2011), copyright by Elsevier

extrapolations from GONG data marking open and closed field lines. With such inevitable differences in the latitude between the measuring spacecraft, rather large uncertainties in the $1 \mathrm{AU}$ measured speed of a particular coronal hole are obtained (see Hofmeister et al. 2018; Owens et al. 2019). Recent studies suggest that solar wind forecasting based on persistence models may work best when using a combination of spacecraft located behind Earth (L5 position or STEREO data from time-varying spacecraft position) and empirical or numerical solar wind modeling (see e.g., Opitz et al. 2010; Temmer et al. 2018; Owens et al. 2019; Bailey et al. 2020).

\subsection{Solar wind structures affecting CME and SEP evolution}

CMEs are known to change shape, accelerate or decelerate, depending on the background solar wind flow properties, as well as may change their propagation direction if encountering other magnetic structures (cf. Sect. 5). From recent results it is found that many coronal fine structures and small scale magnetic flux ropes are embedded in the solar wind (first PSP/WISPR results see Howard et al. 2019). Likewise, PSP in-situ measurements show that low-latitude coronal holes might be an additional source of slow solar wind, causing close to the Sun strong fluctuations in the solar wind flow (Bale et al. 2019). Such local solar wind dynamics, comprising of numerous voids, compact small- to large-scale sized structures ("woodgrain" appearance), clearly have impact on the evolution of a CME and with that complicates forecasting. As example, Fig. 31 shows a disrupted CME front close to the Sun as consequence of an interaction with a high speed solar wind 

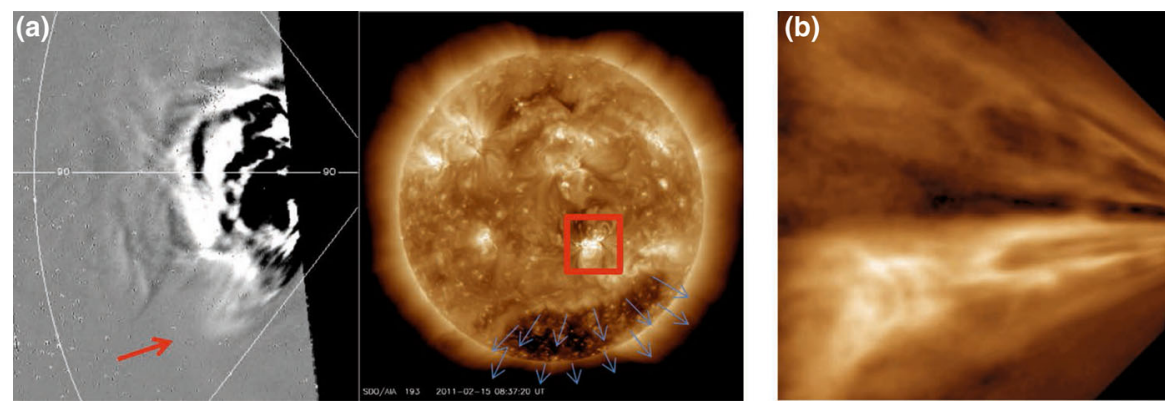

Fig. 31 a Left panel shows a STEREO/HI difference image with the distorted CME front (red arrow). Right panel gives SDO/AIA $193 \AA$ EUV image with the CME source region (red box) and the southern coronal hole. The fast solar wind stream out of the coronal hole (depicted with blue arrows) deforms the CME front. b Highly structured solar wind flow close to the Sun from a STEREO/HI image after computer processing. NASA's Goddard Space Flight Center/Craig DeForest, SwRI

stream emanating from the southern polar coronal hole. The increased speed of the ambient solar wind flow and stretched magnetic field, causes a weaker compression and, hence, deviations from the shape of a circular/elliptic CME front. The structured solar wind in the outer corona is also revealed from specially noise reduced post-processed STEREO/HI image data applying an algorithm to dim the appearance of bright stars and dust (right panel of Fig. 31; cf. DeForest et al. 2018). See also recent results from PSP image data resolving small-scale flux ropes, density structures and fluctuations in solar wind streamers (Howard et al. 2019). Besides adjusting to the ambient solar wind flow speed, CMEs also tend to rotate for adjusting to the ambient magnetic field (e.g., Yurchyshyn et al. 2001, 2009; Vourlidas et al. 2011; Isavnin et al. 2014).

SEPs propagate along the magnetic field lines, hence are directly guided by the interplanetary magnetic field structure. During CME events, SEP path lengths are found to be much longer compared to quiet solar wind conditions (e.g., Masson et al. 2012). For SEPs, which are injected into the legs of a CME, the magnetic path lengths from the Sun to Earth may vary largely, up to a factor of two, depending on the specific width of the CME (Reames 2009). CME geometry as well as kinematics (propagation of shock front) and time-dependent changes play an important role with respect to particle acceleration processes. In that respect, SEPs can be taken as probes as they map the interplanetary magnetic field structure (Reames 2017).

\subsection{Preconditioning of interplanetary space}

The correct simulation of the prevailing background solar wind structures in interplanetary space is important for a reliable CME-SEP forecasting. Using MHD modeling to simulate the solar wind distribution works well for low solar activity. However, increased solar activity changes the magnetic field in the photosphere, which serves as main observational model input, more quickly and propagating CMEs strongly disturb the interplanetary background solar wind making models tend to fail (see e.g., Lee et al. 2009; Gressl et al. 2014). This effect is commonly 
known as preconditioning of interplanetary space, which alters the initial conditions for CME and SEP evolution. It is found that single CME events may disturb the slowly evolving solar wind flow for 2-5 days (Temmer et al. 2017a; Janvier et al. 2019) and most strong preconditioning effects are obtained due to CME-CME or CME-CIR interacting events as they form complex magnetic structures (e.g., Gopalswamy et al. 2000; Burlaga et al. 2002; Harrison et al. 2012; Dumbović et al. 2019). Multiple CME activity is supposed to cause a decrease of density in interplanetary space and a radial stretching of the interplanetary magnetic field. That leads to a low drag force acting on subsequently propagating CMEs (see e.g., Farrugia and Berdichevsky 2004; Maričić et al. 2014). The STEREO-A directed July 23, 2012 event was one of the fastest CMEs ever recorded and propagated over a 1 AU distance in less than 21 hours (Liu et al. 2014). It could be shown that the drag parameter, due to a preceding CME from July 19, 2012, was lowered by one order of magnitude (Temmer and Nitta 2015). If the super-fast CME from July 2012 would have been Earth directed, it would have caused an extreme Space Weather event with an estimated Dst of -600 to -1100 nT (e.g. Ngwira et al. 2014; Baker et al. 2013). Due to increased fluctuations and extended periods of negative $B_{\mathrm{z}}$ most intense geomagnetic storms occur for complex interacting CMEs (e.g., Wang et al. 2003; Farrugia et al. 2006; Xie et al. 2006; Dumbović et al. 2015; Scolini et al. 2020).

For SEPs, the preconditioning that comes from a preceding CME has consequences in the seed population. The presence of a previous CME is found to increase the probability for the subsequent fast CME to be SEP-rich (Gopalswamy et al. 2002, 2004; Kahler and Vourlidas 2005). The amount of particles that can get accelerated is increased for multiple CMEs and the increased turbulence in the interaction region is likely to accelerate the particles more efficiently to higher energies (so-called twin-CME scenario as proposed by Li and Zank 2005). Furthermore, the magnetic structure configuration in the sheath region for ICMEs is changed as the CME propagates in interplanetary space, by which the magnetic connectivity is altered (see also review by Kilpua et al. 2017, on CME sheath regions). Interacting CMEs are also found to be more often related to

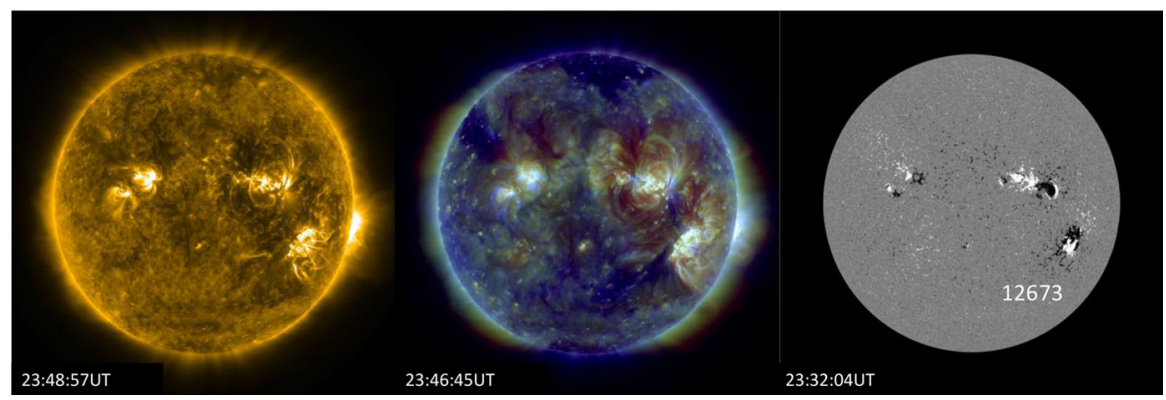

Fig. 32 High-resolution SDO/AIA multi-wavelength imagery from September 6, 2017 (left: $171 \AA$, middle: composite from 211-193-171 $\AA$ ) and SDO/HMI line-of-sight magnetic field (right panel). The prominent active region NOAA 12673 caused the strongest eruptions during solar cycle 24 including SEPs. Courtesy of AIA team, taken from http://suntoday.lmsal.com 
widespread SEPs that can be observed all around the Sun using multiple viewpoints (e.g., Dresing et al. 2016; Gómez-Herrero et al. 2017). For more details on CMECME interaction and SEPs, I refer to the review by Lugaz et al. (2017b).

Over the solar cycle, the CME occurrence rate lies on average in the range of 0.3 per day during solar minimum phase and about 4-5 per day during solar maximum phase (e.g., St Cyr et al. 2000). With average CME transit times from Sun to 1 AU of the order of 1-4 days we can assume that during times of increased solar activity CME-CME interaction happens rather frequently. Reliably modeling these dynamic conditions in interplanetary space is, therefore, key for improving Space Weather forecasting capabilities.

\section{The chain of action on the example of the September 2017 events}

The September 2017 activity phenomena are so far the most well studied strong Space Weather events in our modern space research era. Therefore, the chain of action can be described in great detail, especially with respect to the solar surface signatures and deduced parameters. The multiple disturbances can be well connected from Sun to Earth (and also up to Mars) and show how complex interactions lead to preconditioning effects and strong geoffectiveness.

A strong emergence of magnetic flux in the southern hemisphere of the Sun rapidly led to the development of active region NOAA 12673 into a complex $\alpha \beta \gamma$ magnetic field configuration. As consequence, between September 4-10, 2017 that active region released a series of major flare events, which were actually the largest in more than a decade. These multiple events caused very strong geomagnetic disturbances with a minimum Dst of $-142 \mathrm{nT}$ on September 7, 2017. Additional minor storms were produced by high speed solar wind streams that arrived together with the transient events. Figure 32 gives EUV image data taken with SDO/AIA on

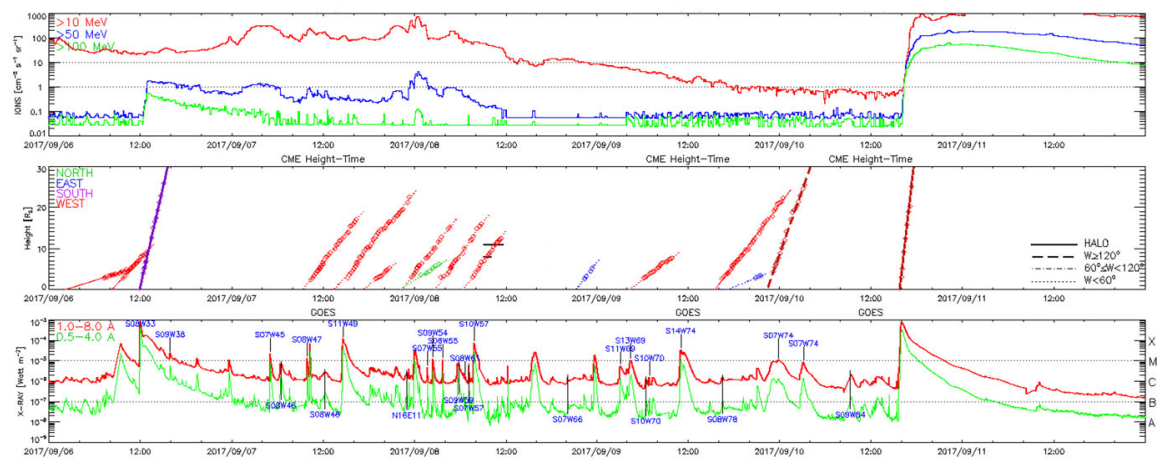

Fig. 33 September 2017 flare-CME-SEP series taken and adapted from the CDAW catalogue (protonheight/time-X-ray plots_PHTX_can be found under https://cdaw.gsfc.nasa.gov/CME_list). Top panel shows solar energetic particle events for protons in the GOES energy channels $>10,>50$ and $>100 \mathrm{MeV}$. Middle panel gives the CME height-time profile as measured from LASCO (colors give the main propagation direction-see legend to the left in the middle panel). Bottom panel gives the GOES flare SXR emission disk integrated over the wavelength ranges $0.5-4.0$ and $1.0-8.0 \AA$ 
September 6, 2017 in different wavelength ranges showing the hot corona from about 0.6 to $2 \mathrm{MK}$. The wavelength ranges cover $171 \AA$ (left panel), and with a triple-filter $211 \AA$ (red), $193 \AA$ (green), and $171 \AA$ (blue) to highlight different temperatures (middle panel). The line-of-sight magnetogram for the same day taken with SDO/HMI reveals the magnetic field in the photosphere (right panel).

Figure 33 gives a combined overview on the series of activity pulses covering the two major flare-CME-SEP events during September 6-10, 2017. In total, active region NOAA 12673 produced an intense solar storm period revealing five X-class flares and $39 \mathrm{M}$-class flares (including the two largest flares from solar cycle 24, the X9.3 flare on September 6, 2017 and the X8.2 flare on September 10, 2017). The first SEP event was measured in the GOES channels at 1AU over September 6, 2017 12:15UT-September 7, 2017 23:25UT and is related to the halo CME that occurred on the Sun on September 6, 2017 12:24UT (first observation in LASCO/C2) with a projected speed of $1570 \mathrm{~km} / \mathrm{s}$ over the coronagraph field of view. The CME has the source region location coordinates S08W33 and is launched together with a flare that started on September 6, 2017 11:53UT and reached X9.3 class in the measured

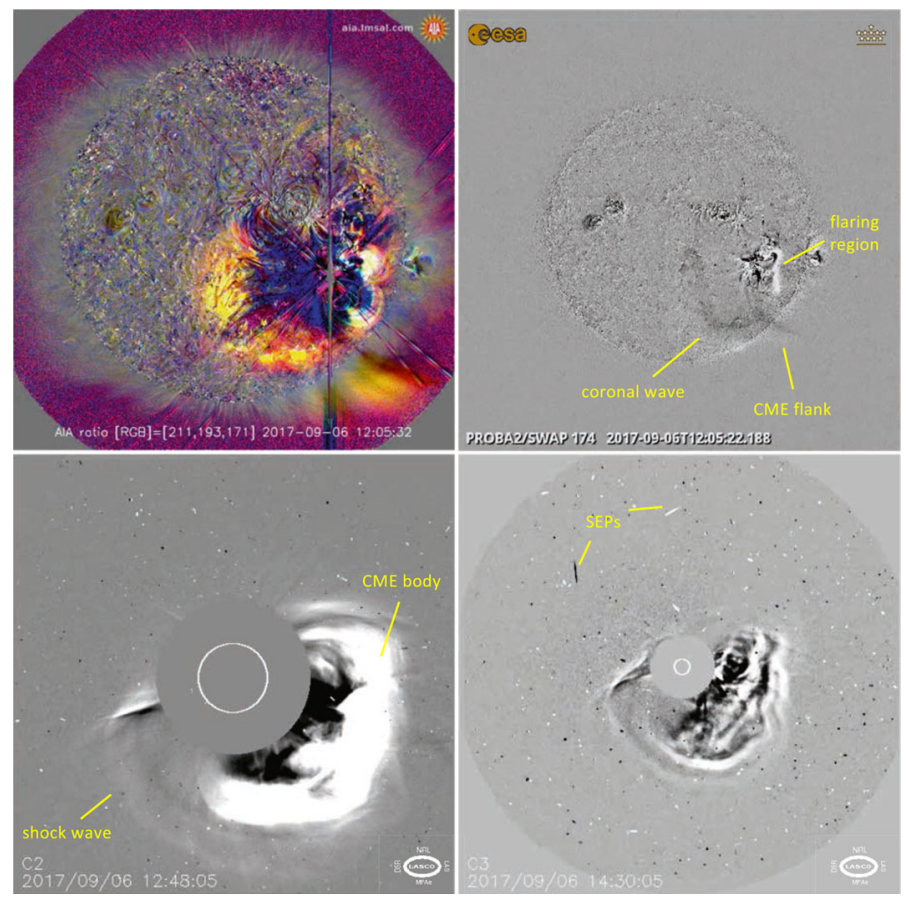

Fig. 34 Top panels: EUV images from September 6, 2017 observed with SDO AIA (running ratio of composite image data) and Proba-2/SWAP (difference image of 171 A data). Bottom panels: LASCO/C2 and $\mathrm{C} 3$ coronagraphs covering a field of view up to 30 solar radii. The generated SEPs are accelerated to relativistic speeds producing spikes in the image data ("snowstorm" effect). This event was the first flare event in a sequence of X-class flares on 6, 7, and 10 September 2017 causing strong disturbances at Earth and Mars. Note that the field of view of Proba-2 is larger compared to AIA and that due to different CCD techniques no saturation effects are visible in Proba-2. Movies for each panel are available in the online supplement 
GOES SXR flux. The second flare-CME-SEP event occurred September 10, 2017 (SEP: September 10, 2017 16:25UT-September 11, 2017 11:40UT; halo CME: September 10, 2017 16:00 with 1490 km/s; flare: X8.2 class on September 10, 2017 15:35UT) with the source region located behind the west limb. For both events long-duration high-energy gamma-ray emission was detected by the Fermi-Large Area Telescope, having durations exceeding 15 hours and with that being the third and fifth largest among all detected (Longo et al. 2017; Gopalswamy et al. 2018a; Omodei et al. 2018).

Figure 34 impressively shows the manifestation of the eruptive event from September 6, 2017 around 12 UT in solar observations using EUV image and whitelight coronagraph data. To make changes visible, different techniques are used, such as a running ratio as applied on the SDO/AIA composite image and difference images as applied on the Proba-2/SWAP $171 \AA$ or on the LASCO white-light data. SDO and Proba-2 processed images nicely reveal the flare component (X9.3 class), a coronal surface wave that was ignited, and the CME flank on-disk as well above the limb visible in EUV due to compression and heating of the plasma. The off-disk counterpart of the coronal wave is observed in white-light as the shock wave surrounding the CME body. In white-light coronagraph data from LASCO C2 and C3 the CME is detected as partial halo event. The projected CME speed over the field of view up to 20 solar radii was measured from LASCO data with $\sim 1500 \mathrm{~km} / \mathrm{s}$. SEPs that are produced by relativistic protons hit the CCD camera of LASCO within minutes and generate the well-known "snowstorm" effect. Heavy snowstorms strongly disturb the image quality which may complicate the analysis of halo events (especially during times of increased solar activity).

Figure 35 shows for the September 6, 2017 event the associated dimming region and post-eruptive arcade together with the magnetic field. The core dimmings are
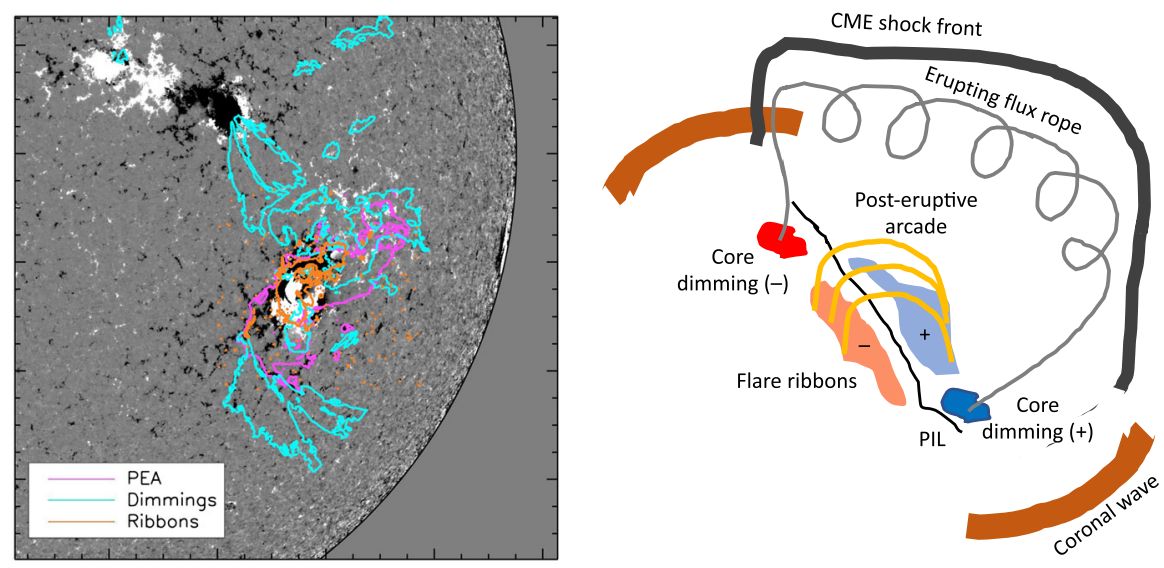

Fig. 35 Left panel: location of flare ribbons (orange contour), dimming areas (cyan contour) and posteruptive arcade (PEA, magenta contour) derived for the eruptive event from September 6, 2017. The greyscale background HMI line-of-sight magnetogram is scaled to $\pm 100 \mathrm{G}$ with black and white representing negative and positive polarities, respectively (adapted from Scolini et al. 2020). Right panel: cartoon giving the relation between flare, CME erupting flux rope, coronal wave and dimming areas 


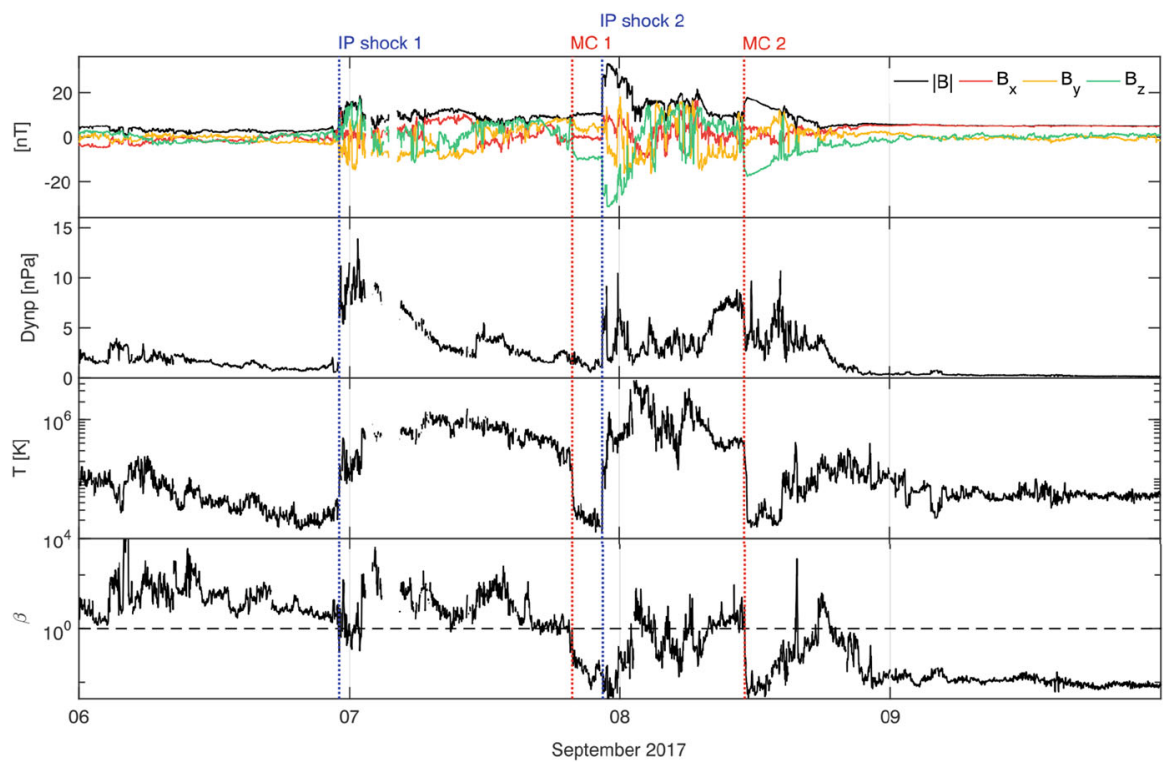

Fig. 36 Interacting in-situ signatures from September 6-9, 2017 caused by the multiple CME events. Magnetic field and plasma data are given from WIND at L1. Top to bottom: Total magnetic field and vector components, dynamical pressure, proton temperature, and plasma-beta. Image reproduced with permission from Werner et al. (2019), copyright by AGU

accompanied by secondary dimmings covering larger areas. The cartoon to the right in Fig. 35 depicts the relation between different features of an eruptive event and associated solar surface signatures. For example, measurements of the post-eruptive arcade (PEA) areas and their underlying magnetic field were taken to derive the reconnected flux to feed CME propagation models using magnetized CMEs (Scolini et al. 2020). The orientation of the PEA with respect to the underlying magnetic field, revealed the handedness of the flux rope. Coronal waves initiated close to the eruption side, hint towards the main compression direction of the eruption, hence, a southward propagation direction of the associated CME (confirmed by the coronagraph images; cf. Fig. 34). Therefore, monitoring surface structures gives additional (and early) information about a potential Space Weather event that might affect Earth, and moreover, provides valuable input for modeling efforts.

The multiple CME events preconditioned interplanetary space and were interacting, which intensified their geomagnetic effects. Figure 36 shows over September 6-9, 2017 the complex in-situ signatures revealing multiple shocks and magnetic ejecta regions. Note that the shock of ICME2 propagated into the magnetic structure of ICME1. These so-called "shock-in-a-cloud" events are found to cause stronger geomagnetic responses than isolated geoeffective CMEs (Lugaz et al. 2015). For the September 2017 events, the shock compression might have enhanced the geoeffectiveness by a factor of 2 (Shen et al. 2018).

As the active region rotates close to the West limb of the Sun, the event from September 10 occurred as partially occulted and, because of the favorable magnetic 


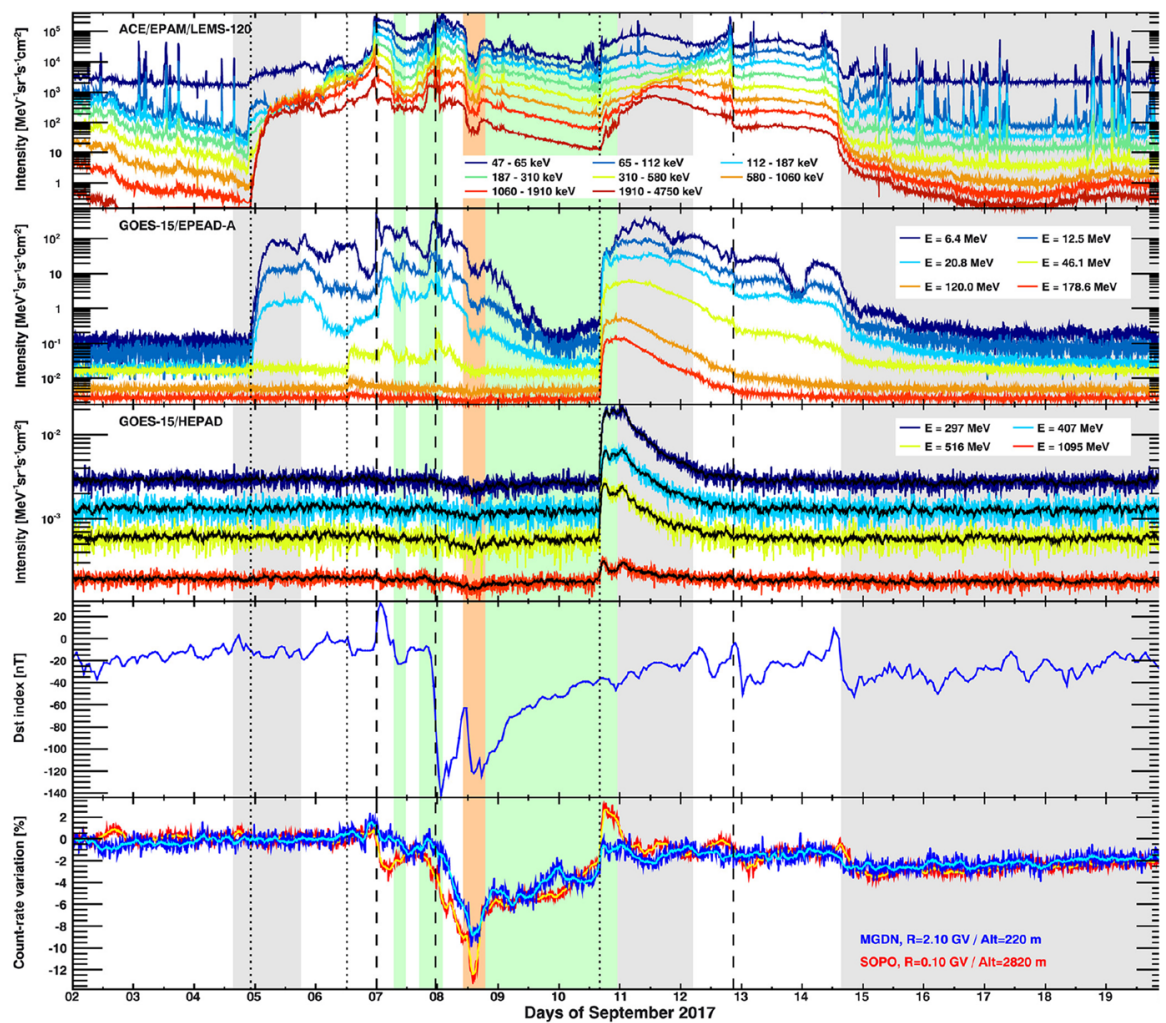

Fig. 37 Proton intensities measured by ACE/EPAM, GOES/EPEAD, and GOES/HEPAD. Dst index and count rate variations registered by SOPO and MGDN neutron monitor stations. The vertical dotted and dashed lines mark the onset of the SEP events and the time of the shocks, respectively. The green, orange, and gray areas indicate the periods of the ICME, magnetic cloud, and high speed streams, respectively. Image reproduced with permission from Bruno et al. (2019), copyright by AGU

connectivity, generated a GLE. Due to the eruption site located close to the limb, measurements of the CME kinematics were less strongly affected by projection effects. From EUV observations of the wide field of view Solar Ultraviolet Imager on board the GOES-16 spacecraft exceptionally high values for the CME acceleration and speed were derived, revealing the huge expansion in both, radial and lateral direction (Gopalswamy et al. 2018b; Seaton and Darnel 2018; Veronig et al. 2018). This caused the initiation of a coronal wave propagating over the entire solar surface (Liu et al. 2018). Figure 37 gives the SEP proton fluxes measured near Earth (ACE, GOES) together with the geomagnetic Dst index and neutron monitor profiles showing the Forbush decrease related to the arrival of the closed magnetic structure (for more details see Bruno et al. 2019).

The September events did not only affect the Earth, but were also registered at Mars. Energetic particles were observed by MAVEN orbiter, and on the surface by the Curiosity Mars rover (e.g., Hassler et al. 2018). Many further aspects of the 
September 2017 activity period are well represented in a collection of publications in the AGU Space Weather journal. ${ }^{24}$

\section{Space weather forecasting models}

"Since all models are wrong the scientist must be alert to what is importantly wrong. It is inappropriate to be concerned about mice when there are tigers abroad." by Box (1976).

For comprehensive investigations of the structure of interplanetary space, propagating transient events, and magnetic connectivity, we need to rely on modeling efforts. Having only scarcely distributed single point in-situ measurements and the lack of plasma and magnetic field information derived by applying remote sensing imaging techniques, we are clearly limited in our ability to assess the performance and reliability of these models hindering improvements. Nevertheless, the close collaboration between model developers and observational community is a need to push forward our understanding of CME-solar wind coupling and interaction processes in interplanetary space. Also on kinetic scales, particle acceleration processes and trigger mechanisms of flare-CME-SEP events need to be supported by modeling, as from observational data the information are not sufficient to make conclusive interpretations about the underlying physics. In recent years substantial progress has been made in efficiently combining models with observations. Data assimilation techniques are known to hugely improve operational forecasting models, as observational data are incorporated in a self consistent way into numerical models for increasing the model accuracy. Examples about the application to solar data can be found in e.g., Schrijver and De Rosa (2003), Arge et al. (2010). In heliospheric physics this method is difficult to apply due to the relatively sparse observations. Recent efforts comprise in-situ measurements from $1 \mathrm{AU}$ to update and improve inner-boundary conditions of solar wind models (see e.g., Lang and Owens 2019).

Forecasting flares and SEPs with a lead time of at least a few hours is related to the forecasting of the evolution of active regions producing eruptive solar flare events. However, that means to estimate the emergence of magnetic flux from below the photosphere that is not accessible from direct observations. Therefore, statistical relations between photospheric magnetic field characteristics of active regions and flare occurrence are usually used for solar flare forecasting. More precisely, it is the time evolution of the magnetic parameters that plays a major role, but is very complex to derive. The prediction of major flares seems to be more easily achievable compared to flares of medium to low energy, however, the uncertainties coming from the statistical approach are rather large preventing from more accurate single event prediction (see e.g., Schrijver 2007; Georgoulis and Rust 2007; Bloomfield et al. 2012). Real-time solar flare forecasting is provided by e.g., the EU project Flarecast $^{25}$ with a fully automated system and the NASA/CCMC flare

\footnotetext{
${ }^{24}$ https://agupubs.onlinelibrary.wiley.com/doi/toc/10.1002/(ISSN)1542-7390.SW-SEPT2017.

25 http://flarecast.eu.
} 
forecasting scoreboard provides a platform to test different forecasting methods fostering further development of the available algorithms. ${ }^{26}$ In general, having standardized metrics is a necessity in order to reliably validate and cross-check the performance of the different flare forecasting tools (Barnes and Leka 2008). See also a review on the origin, early evolution and predictability of solar eruptions by Green et al. (2018).

For forecasting CME arrival times and impact, a vast amount of models is currently available in various levels of complexity (see, e.g., Table 1 in Riley et al. 2018). Simple models, such as empirical relations between CME transit time and speed from statistical results provide tools to derive an average behavior of CME propagation in interplanetary space (Gopalswamy et al. 2001a). The forecasting accuracy can be significantly improved, when using observational data that can track the CME kinematics to beyond a distance of 50 solar radii (e.g., using image data from STEREO/HI, or radio IPS) where the CME is assumed to evolve in a linear way (see Colaninno et al. 2013; Rollett et al. 2016; Hess and Zhang 2017). Several analytical models include the physics of drag force (viscous, aerodynamic, hybrid - that means a linear or quadratic relation between solar wind and CME speed difference) that a CME experiences in interplanetary space. A widely used analytical model is the drag-based-model (DBM; see Vršnak et al. 2013). It applies the aerodynamic drag as analogon for the MHD drag force exerted on the CME embedded flux rope. More sophisticated are numerical MHD models such as e.g., EUHFORIA (Pomoell and Poedts 2018), ENLIL (Odstrčil and Pizzo 1999), CORHEL (Riley et al. 2012) or SUSANOO (Shiota and Kataoka 2016). Besides simulating transient events, MHD models also cover the variation in the background solar wind (see also Arge and Pizzo 2000). A compilation of data, services and tools can be found at CDPP (Plasma physics data center in France). ${ }^{27}$ For CMEs, the disentanglement between shock and magnetic ejecta is an important issue highly relevant for forecasting. Numerical models usually use a simple pressure pulse to ignite a CME shock front, but do not include the magnetic structure. There are recent efforts of magnetized CMEs incorporating the observed reconnected magnetic flux at the Sun as model input parameter for the CME flux rope (e.g. Scolini et al. 2019; Singh et al. 2019).

For validation purposes and for increasing the awareness of the limitations of the accuracy of the model results, the uncertainties of observational model input data need to be quantified. Riley et al. (2018) summarized hit and miss statistics from the CCMC scoreboard, ${ }^{28}$ a CME prediction board that gives the possibility to use different models in real-time forecasting (crude facts approach that challenges models and their users). Metadata and metrics are suggested to give the community a common base for an objective inter-comparison of their models (Verbeke et al. 2019). A review on the current status and open issues on CME propagation and forecasting methods is given by Vourlidas et al. (2019).

\footnotetext{
26 https://ccmc.gsfc.nasa.gov/challenges/flare.php.

27 http://cdpp.irap.omp.eu.

28 https://kauai.ccmc.gsfc.nasa.gov/CMEscoreboard/.
} 
Compared to the prediction of CME arrival times and impact speeds, SEP forecasting is a more tricky issue as the accelerated particles propagate with fractions of the speed of light and the lead time is of only a few minutes. Several models are available, mostly using empirical relations based on statistical relations with CME-flare locations or type II radio burst occurrence at decametrichectometric (DH) wavelengths. Current models cover e.g., PROTONS (Balch 2008), PPS (Kahler et al. 2007), ESPERTA (Laurenza et al. 2009), FORSPEF (Anastasiadis et al. 2017) as well as physics based models such as e.g., SOLPENCO (Aran et al. 2006) or HESPERIA (Malandraki and Crosby 2018, and references therein). Incorporating CME characteristics is difficult as the real-time coronagraph image data, from which usually the CME parameters are derived, are delivered with some delay. Nevertheless, the SEPForecast tool resulting from the EU project COMESEP $^{29}$ also uses CME parameters as model input (Dierckxsens et al. 2015). The application of solar surface proxies for some CME parameters might overcome that drawback. A detailed comparison of false alarm rates for SEP forecasting is presented in e.g., Alberti et al. (2017). The current status of forecasting and nowcasting of SEPs and open questions is given in a review by Anastasiadis et al. (2019).

Further approaches for improving forecasting purposes also cover ensemble models incorporating the uncertainties in the observational data (e.g., Lee et al. 2013; Mays et al. 2015; Dumbović et al. 2018; Amerstorfer et al. 2018), and machine learning techniques (see e.g., Camporeale 2019). In addition to methods covering large statistics, case studies that model Space Weather events from Sun to Earth, give a wealth of detailed information from which we can hugely improve our understanding of flare-CME-SEP events. Community centers like ESA/VSWMC ${ }^{30}$ or NASA/CCMC ${ }^{31}$ cover the increased need of computational power and appropriate IT infrastructure and provide a platform for models to be tested and actually used. Such platforms are also the driveway for R2O (research to operation) activities and where scientists and users meet. A collection of Space Weather tools is presented at the ESA/SSA website, including services from the European Expert Service Centers and their individual Expert Groups (cf. Fig. 38).

\section{Concluding remarks}

Nowadays, the term Space Weather covers basic research as well as application and is a platform for strong interdisciplinary research bringing the domains of solar-, heliospheric, and geo-physics closer together. The real-time forecasting results of flare-CME-CIR-SEP events at Earth using operational tools reveal that we still face large uncertainties. The reason is on the one hand the huge complexity of the solar phenomena and their unknown coupling processes and propagation behavior in interplanetary space. On the other hand, there is a clear lack of accurate enough

\footnotetext{
29 http://comesep.aeronomy.be.

30 https://esa-vswmc.eu.

31 https://ccmc.gsfc.nasa.gov.
} 


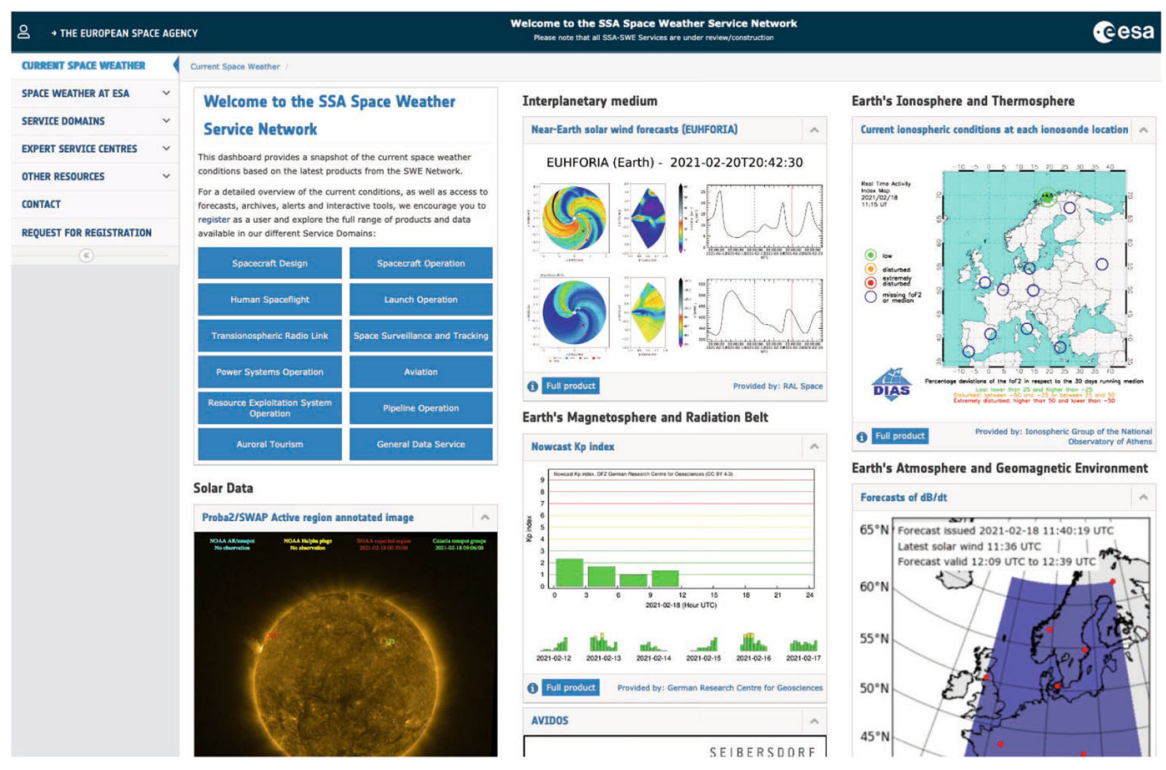

Fig. 38 Webpage of the SSA Space Weather Service Network where Expert Service Centers provide their tools and services covering Space Weather forecast and overview from the solar-heliosphere perspective to space radiation, ionospheric and geomagnetic conditions. ESA/SSA: http://swe.ssa.esa.int

measurements to properly feed the available models. The source of high errors and inaccuracies in the measurements is due to extraction of remote sensing data which are affected by projection effects, idealized assumptions of magnetic field extrapolations (lack of coronal magnetic field measurements) and 3D reconstructions, as well as localized in-situ measurements making it tricky to validate all the results. For understanding and forecasting the geoeffectiveness of Earth affecting Space Weather events, a far bigger challenge for the future is the modeling of the interplanetary magnetic field orientation and variation.

A clear advantage is given by the combination of several instruments having multiple views on the Sun covering different longitudes and latitudes. STEREO-A is still operational providing data material from varying angles. PSP will be the first spacecraft that comes as close to the Sun as 10 solar radii and aims to answer questions about the origin of the solar wind, how it is accelerated or about SEP acceleration and transport processes. The mission already gathered unprecedented data enabling new interpretation and finding on the source regions of the solar wind. Solar Orbiter revealed first data in July 2020 and will study the solar surface and its magnetic field in great detail. The spacecraft will have a highly elliptical orbit to progressively move to a more inclined orbit out of ecliptic. That perspective will give new insight onto the polar regions of the Sun and is expected to improve magnetic field modeling and to better understand the solar wind from coronal holes in the polar regions. Conjunctions with other active missions (such as STEREO, L1 missions, MAVEN), complemented by ground-based instruments, will give unique possibilities to investigate the evolution of solar flares, CMEs, or SEPs, as well as 
coronal holes and solar wind high speed streams. Future missions, such as the ESA Lagrange mission to L5 (launch is planned for 2027) or polar missions, as the proposed Solaris Solar Polar Mission (Hassler et al. 2020), will provide more valuable data and fill the gaps in our understanding of magnetic field connectivity and coupling processes between open and closed magnetic field structures in interplanetary space.

We are facing challenging and exciting times having a wealth of data at hand and promising future missions yet to come. Many of the currently available data are well prepared in ready-to-use catalogues, waiting to be explored. However, we still need to obtain data from L4/L5 Lagrange points revealing the necessary side views on a regular basis to fully track eruptions with low projection effects and for gaining a better understanding of their characteristics that affect Earth. Moreover, we need to improve solar wind models to be used in MHD simulations for properly determining CME propagation. With that we will successfully enhance the reliability of Space Weather forecasting tools and models.

Supplementary Information The online version contains supplementary material available at https://doi. org/10.1007/s41116-021-00030-3.

Acknowledgements I would like to thank Stephan G. Heinemann for his support in generating the BibTeX entries. Some images were created using the ESA and NASA funded Helioviewer Project. LASCO images courtesy of SOHO/LASCO consortium. SOHO is a project of international cooperation between ESA and NASA. SDO images courtesy of SDO/AIA consortium. STEREO images courtesy of STEREO/SECCHI consortium. SWAP images courtesy of PROBA2/SWAP consortium. PROBA2 is a project of the Centre Spatial de Liège and the Royal Observatory of Belgium funded by the Belgian Federal Science Policy Office (BELSPO) and ESA.

Open Access This article is licensed under a Creative Commons Attribution 4.0 International License, which permits use, sharing, adaptation, distribution and reproduction in any medium or format, as long as you give appropriate credit to the original author(s) and the source, provide a link to the Creative Commons licence, and indicate if changes were made. The images or other third party material in this article are included in the article's Creative Commons licence, unless indicated otherwise in a credit line to the material. If material is not included in the article's Creative Commons licence and your intended use is not permitted by statutory regulation or exceeds the permitted use, you will need to obtain permission directly from the copyright holder. To view a copy of this licence, visit http:// creativecommons.org/licenses/by/4.0/.

\section{References}

Acuña MH, Curtis D, Scheifele JL, Russell CT, Schroeder P, Szabo A, Luhmann JG (2008) The STEREO/IMPACT magnetic field experiment. Space Sci Rev 136(1-4):203-226. https://doi.org/10. 1007/s11214-007-9259-2

Akiyama S, Gopalswamy N, Yashiro S, Mäkelä P (2013) A study of coronal holes observed by SoHO/ EIT and the Nobeyama Radioheliograph. Publ Astron Soc Jpn 65:S15. https://doi.org/10.1093/pasj/ 65.sp1.S15

Al-Haddad N, Nieves-Chinchilla T, Savani NP, Möstl C, Marubashi K, Hidalgo MA, Roussev II, Poedts S, Farrugia CJ (2013) Magnetic field configuration models and reconstruction methods for interplanetary coronal mass ejections. Sol Phys 284(1):129-149. https://doi.org/10.1007/s11207013-0244-5. arXiv:1209.6394 [astro-ph.SR]

Alberti T, Laurenza M, Cliver EW, Storini M, Consolini G, Lepreti F (2017) Solar activity from 2006 to 2014 and short-term forecasts of solar proton events using the ESPERTA model. Astrophys J 838(1):59. https://doi.org/10.3847/1538-4357/aa5cb8 
Amerstorfer T, Möstl C, Hess P, Temmer M, Mays ML, Reiss MA, Lowrance P, Bourdin PA (2018) Ensemble prediction of a halo coronal mass ejection using heliospheric imagers. Space Weather. https://doi.org/10.1029/2017SW001786

Anastasiadis A, Papaioannou A, Sandberg I, Georgoulis M, Tziotziou K, Kouloumvakos A, Jiggens P (2017) Predicting flares and solar energetic particle events: the FORSPEF tool. Sol Phys 292(9):134. https://doi.org/10.1007/s11207-017-1163-7

Anastasiadis A, Lario D, Papaioannou A, Kouloumvakos A, Vourlidas A (2019) Solar energetic particles in the inner heliosphere: status and open questions. Philos Trans R Soc Lond Ser A 377(2148):20180100. https://doi.org/10.1098/rsta.2018.0100

Aran A, Sanahuja B, Lario D (2006) SOLPENCO: a solar particle engineering code. Adv Space Res 37(6):1240-1246. https://doi.org/10.1016/j.asr.2005.09.019

Arden WM, Norton AA, Sun X (2014) A "breathing"' source surface for cycles 23 and 24. J Geophys Res 119(3):1476-1485. https://doi.org/10.1002/2013JA019464

Arge CN, Pizzo VJ (2000) Improvement in the prediction of solar wind conditions using near-real time solar magnetic field updates. J Geophys Res 105(A5):10465-10480. https://doi.org/10.1029/ 1999JA000262

Arge CN, Henney CJ, Koller J, Compeau CR, Young S, MacKenzie D, Fay A, Harvey JW (2010) Air force data assimilative photospheric flux transport (ADAPT) model. In: Maksimovic M, Issautier K, Meyer-Vernet N, Moncuquet M, Pantellini F (eds) Twelfth international solar wind conference. AIP conference series, vol 1216. American Institute of Physics, pp 343-346. https://doi.org/10.1063/1. 3395870

Aschwanden MJ (2011) Solar stereoscopy and tomography. Living Rev Sol Phys 8:5. https://doi.org/10. 12942/lrsp-2011-5

Aschwanden MJ (2019) New millennium solar physics, Astrophysics and Space Science Library, vol 458. Springer, Cham. https://doi.org/10.1007/978-3-030-13956-8

Aschwanden MJ, Caspi A, Cohen CMS, Holman G, Jing J, Kretzschmar M, Kontar EP, McTiernan JM, Mewaldt RA, O'Flannagain A, Richardson IG, Ryan D, Warren HP, Xu Y (2017) Global Energetics of Solar Flares. V. Energy closure in flares and coronal mass ejections. Astrophys J 836(1):17. https://doi.org/10.3847/1538-4357/836/1/17arXiv:1701.01176 [astro-ph.SR]

Bailey RL, Möstl C, Reiss MA, Weiss AJ, Amerstorfer UV, Amerstorfer T, Hinterreiter J, Magnes W, Leonhardt R (2020) Prediction of Dst during solar minimum using in situ measurements at L5. Space Weather 18(5):e02424. https://doi.org/10.1029/2019SW002424. arXiv:2005.00249 [astroph.SR]

Baker DN, Hones JEW, Payne JB, Feldman WC (1981) A high time resolution study of interplanetary parameter correlations with AE. Geophys Res Lett 8(2):179-182. https://doi.org/10.1029/ GL008i002p00179

Baker DN, Li X, Pulkkinen A, Ngwira CM, Mays ML, Galvin AB, Simunac KDC (2013) A major solar eruptive event in July 2012: defining extreme space weather scenarios. Space Weather 11(10):585-591. https://doi.org/10.1002/swe.20097

Balch CC (2008) Updated verification of the Space Weather Prediction Center's solar energetic particle prediction model. Space Weather 6(1):S01001. https://doi.org/10.1029/2007SW000337

Bale SD, Badman ST, Bonnell JW, Bowen TA, Burgess D, Case AW, Cattell CA, Chandran BDG, Chaston CC, Chen CHK, Drake JF, de Wit TD, Eastwood JP, Ergun RE, Farrell WM, Fong C, Goetz K, Goldstein M, Goodrich KA, Harvey PR, Horbury TS, Howes GG, Kasper JC, Kellogg PJ, Klimchuk JA, Korreck KE, Krasnoselskikh VV, Krucker S, Laker R, Larson DE, MacDowall RJ, Maksimovic M, Malaspina DM, Martinez-Oliveros J, McComas DJ, Meyer-Vernet N, Moncuquet M, Mozer FS, Phan TD, Pulupa M, Raouafi NE, Salem C, Stansby D, Stevens M, Szabo A, Velli M, Woolley T, Wygant JR (2019) Highly structured slow solar wind emerging from an equatorial coronal hole. Nature 576(7786):237-242. https://doi.org/10.1038/s41586-019-1818-7

Balmaceda LA, Vourlidas A, Stenborg G, Dal Lago A (2018) How reliable are the properties of coronal mass ejections measured from a single viewpoint? Astrophys J 863(1):57. https://doi.org/10.3847/ $1538-4357 /$ aacff8

Balmaceda LA, Vourlidas A, Stenborg G, St Cyr OC (2020) On the expansion speed of coronal mass ejections: implications for self-similar evolution. Sol Phys 295(8):107. https://doi.org/10.1007/ s11207-020-01672-6

Banaszkiewicz M, Axford WI, McKenzie JF (1998) An analytic solar magnetic field model. Astron Astrophys 337:940-944 
Barnes D, Davies JA, Harrison RA, Byrne JP, Perry CH, Bothmer V, Eastwood JP, Gallagher PT, Kilpua EKJ, Möstl C, Rodriguez L, Rouillard AP, Odstrčil D (2019) CMEs in the heliosphere: II. A statistical analysis of the kinematic properties derived from single-spacecraft geometrical modelling techniques applied to CMEs detected in the heliosphere from 2007 to 2017 by STEREO/HI-1. Sol Phys 294(5):57. https://doi.org/10.1007/s11207-019-1444-4

Barnes G, Leka KD (2008) Evaluating the performance of solar flare forecasting methods. Astrophys J Lett 688(2):L107. https://doi.org/10.1086/595550

Baumgartner C, Thalmann JK, Veronig AM (2018) On the factors determining the eruptive character of solar flares. Astrophys J 853(2):105. https://doi.org/10.3847/1538-4357/aaa243. arXiv:1712.05106 [astro-ph.SR]

Bein BM, Berkebile-Stoiser S, Veronig AM, Temmer M, Muhr N, Kienreich I, Utz D, Vršnak B (2011) Impulsive acceleration of coronal mass ejections. I. Statistics and coronal mass ejection source region characteristics. Astrophys J. https://doi.org/10.1088/0004-637X/738/2/191

Bein BM, Berkebile-Stoiser S, Veronig AM, Temmer M, Vršnak B (2012) Impulsive acceleration of coronal mass ejections. II. Relation to soft X-ray flares and filament eruptions. Astrophys J. https:// doi.org/10.1088/0004-637X/755/1/44

Bein BM, Temmer M, Vourlidas A, Veronig AM, Utz D (2013) The height evolution of the "true", coronal mass ejection mass derived from STEREO COR1 and COR2 observations. Astrophys J. https://doi.org/10.1088/0004-637X/768/1/31

Bemporad A, Mancuso S (2011) Identification of super- and subcritical regions in shocks driven by coronal mass ejections. Astrophys J Lett 739(2):L64. https://doi.org/10.1088/2041-8205/739/2/L64. arXiv:1108.3783 [astro-ph.SR]

Benz AO (2017) Flare observations. Living Rev Sol Phys 14:2. https://doi.org/10.1007/s41116-016-00043

Billings DE (1959) Distribution of matter with temperature in the emission corona. Astrophys J 130:961. https://doi.org/10.1086/146785

Bisi MM, Breen AR, Jackson BV, Fallows RA, Walsh AP, Mikić Z, Riley P, Owen CJ, Gonzalez-Esparza A, Aguilar-Rodriguez E, Morgan H, Jensen EA, Wood AG, Owens MJ, Tokumaru M, Manoharan PK, Chashei IV, Giunta AS, Linker JA, Shishov VI, Tyul'bashev SA, Agalya G, Glubokova SK, Hamilton MS, Fujiki K, Hick PP, Clover JM, Pintér B (2010) From the Sun to the Earth: the 13 May 2005 coronal mass ejection. Sol Phys 265(1-2):49-127. https://doi.org/10.1007/s11207-010-9602-8

Bloomfield DS, Higgins PA, McAteer RTJ, Gallagher PT (2012) Toward reliable benchmarking of solar flare forecasting methods. Astrophys J Lett 747(2):L41. https://doi.org/10.1088/2041-8205/747/2/ L41. arXiv:1202.5995 [astro-ph.SR]

Borovsky JE, Denton MH, Smith CW (2019) Some properties of the solar wind turbulence at 1 AU statistically examined in the different types of solar wind plasma. J Geophys Res 124(4):2406-2424. https://doi.org/10.1029/2019JA026580

Bosman E, Bothmer V, Nisticò G, Vourlidas A, Howard RA, Davies JA (2012) Three-dimensional properties of coronal mass ejections from STEREO/SECCHI observations. Sol Phys 281(1):167-185. https://doi.org/10.1007/s11207-012-0123-5

Bothmer V, Schwenn R (1998) The structure and origin of magnetic clouds in the solar wind. Ann Geophys 16(1):1-24. https://doi.org/10.1007/s00585-997-0001-X

Bothmer V, Daglis IA, Bogdan TJ (2007) Space weather: physics and effects. Phys Today 60(12):59. https://doi.org/10.1063/1.2825074

Bougeret JL, Goetz K, Kaiser ML, Bale SD, Kellogg PJ, Maksimovic M, Monge N, Monson SJ, Astier PL, Davy S, Dekkali M, Hinze JJ, Manning RE, Aguilar-Rodriguez E, Bonnin X, Briand C, Cairns IH, Cattell CA, Cecconi B, Eastwood J, Ergun RE, Fainberg J, Hoang S, Huttunen KEJ, Krucker S, Lecacheux A, MacDowall RJ, Macher W, Mangeney A, Meetre CA, Moussas X, Nguyen QN, Oswald TH, Pulupa M, Reiner MJ, Robinson PA, Rucker H, Salem C, Santolik O, Silvis JM, Ullrich R, Zarka P, Zouganelis I (2008) S/WAVES: the radio and plasma wave investigation on the STEREO mission. Space Sci Rev 136(1-4):487-528. https://doi.org/10.1007/s11214-007-9298-8

Box GEP (1976) Science and statistics. J Am Stat Assoc 71(356):791-799. https://doi.org/10.2307/ 2286841

Brueckner GE, Howard RA, Koomen MJ, Korendyke CM, Michels DJ, Moses JD, Socker DG, Dere KP, Lamy PL, Llebaria A, Bout MV, Schwenn R, Simnett GM, Bedford DK, Eyles CJ (1995) The Large Angle Spectroscopic Coronagraph (LASCO). Sol Phys 162:357-402. https://doi.org/10.1007/ BF00733434 
Bruno A, Christian ER, de Nolfo GA, Richardson IG, Ryan JM (2019) Spectral analysis of the September 2017 solar energetic particle events. Space Weather 17(3):419-437. https://doi.org/10.1029/ 2018SW002085. arXiv:1902.03969 [physics.space-ph]

Burkepile JT, Hundhausen AJ, Stanger AL, St Cyr OC, Seiden JA (2004) Role of projection effects on solar coronal mass ejection properties: 1 . A study of CMEs associated with limb activity. J Geophys Res 109(A3):A03103. https://doi.org/10.1029/2003JA010149

Burlaga LF, Plunkett SP, St Cyr OC (2002) Successive CMEs and complex ejecta. J Geophys Res 107(A10):1266. https://doi.org/10.1029/2001JA000255

Burt J, Smith B (2012) Deep Space Climate Observatory: the DSCOVR mission. In: 2012 IEEE aerospace conference. IEEE, pp 1-13. https://doi.org/10.1109/AERO.2012.6187025

Byrne JP, Maloney SA, McAteer RTJ, Refojo JM, Gallagher PT (2010) Propagation of an Earth-directed coronal mass ejection in three dimensions. Nat Commun 1(6):74. https://doi.org/10.1038/ ncomms1077. arXiv:1010.0643 [astro-ph.SR]

Cairns IH, Lobzin VV, Donea A, Tingay SJ, McCauley PI, Oberoi D, Duffin RT, Reiner MJ, HurleyWalker N, Kudryavtseva NA, Melrose DB, Harding JC, Bernardi G, Bowman JD, Cappallo RJ, Corey BE, Deshpande A, Emrich D, Goeke R, Hazelton BJ, Johnston-Hollitt M, Kaplan DL, Kasper JC, Kratzenberg E, Lonsdale CJ, Lynch MJ, McWhirter SR, Mitchell DA, Morales MF, Morgan E, Ord SM, Prabu T, Roshi A, Shankar NU, Srivani KS, Subrahmanyan R, Wayth RB, Waterson M, Webster RL, Whitney AR, Williams A, Williams CL (2018) Low altitude solar magnetic reconnection, Type III solar radio bursts, and X-ray emissions. Sci Rep 8:1676. https://doi.org/10. 1038/s41598-018-19195-3

Campbell WH (2003) Introduction to geomagnetic fields, 2nd edn. Cambridge University Press, Cambridge. https://doi.org/10.1017/CBO9781139165136

Camporeale E (2019) The challenge of machine learning in space weather: nowcasting and forecasting. Space Weather 17(8):1166-1207. https://doi.org/10.1029/2018SW002061. arXiv:1903.05192 [physics.space-ph]

Cane HV (2000) Coronal mass ejections and forbush decreases. Space Sci Rev 93:55-77. https://doi.org/ 10.1023/A: 1026532125747

Cane HV, Reames DV, von Rosenvinge TT (1988) The role of interplanetary shocks in the longitude distribution of solar energetic particles. J Geophys Res 93(A9):9555-9567. https://doi.org/10.1029/ JA093iA09p09555

Canfield RC, Hudson HS, McKenzie DE (1999) Sigmoidal morphology and eruptive solar activity. Geophys Res Lett 26(6):627-630. https://doi.org/10.1029/1999GL900105

Cargill PJ (2004) On the aerodynamic drag force acting on interplanetary coronal mass ejections. Sol Phys 221:135-149. https://doi.org/10.1023/B:SOLA.0000033366.10725.a2

Carmichael H (1964) A process for flares. In: Hess WN (ed) The physics of solar flares, NASA Special Publication, vol 50. Washington, DC, p 451

Chen J (2017) Physics of erupting solar flux ropes: coronal mass ejections (CMEs) - recent advances in theory and observation. Phys Plasmas 24(9):090501. https://doi.org/10.1063/1.4993929

Chen J, Krall J (2003) Acceleration of coronal mass ejections. J Geophys Res 108(A11):1410. https://doi. org/10.1029/2003JA009849

Chen PF (2011) Coronal mass ejections: models and their observational basis. Living Rev Sol Phys 8:1. https://doi.org/10.12942/lrsp-2011-1

Ciaravella A, Raymond JC, Kahler SW (2006) Ultraviolet properties of halo coronal mass ejections: doppler shifts, angles, shocks, and bulk morphology. Astrophys J 652(1):774-792. https://doi.org/ $10.1086 / 507171$

Cliver EW, Dietrich WF (2013) The 1859 space weather event revisited: limits of extreme activity. J Space Weather Space Clim 3:A31. https://doi.org/10.1051/swsc/2013053

Cliver EW, Feynman J, Garrett HB (1990) An estimate of the maximum speed of the solar wind, 1938-1989. J Geophys Res 95(A10):17103-17112. https://doi.org/10.1029/JA095iA10p17103

Cliver EW, Kahler SW, Reames DV (2004a) Coronal shocks and solar energetic proton events. Astrophys J 605(2):902-910. https://doi.org/10.1086/382651

Cliver EW, Nitta NV, Thompson BJ, Zhang J (2004b) Coronal shocks of November 1997 revisited: the Cme Type II timing problem. Sol Phys 225(1):105-139. https://doi.org/10.1007/s11207-004-3258-1

Colaninno RC, Vourlidas A (2009) First determination of the true mass of coronal mass ejections: a novel approach to using the two STEREO viewpoints. Astrophys J 698(1):852-858. https://doi.org/10. 1088/0004-637X/698/1/852. arXiv:0903.4344 [astro-ph.SR] 
Colaninno RC, Vourlidas A, Wu CC (2013) Quantitative comparison of methods for predicting the arrival of a coronal mass ejections at Earth based on multiview imaging. J Geophys Res 118(11):6866-6879. https://doi.org/10.1002/2013JA019205

Cranmer SR, Winebarger AR (2019) The properties of the solar corona and its connection to the solar wind. Annu Rev Astron Astrophys 57:157-187. https://doi.org/10.1146/annurev-astro-091918104416. arXiv:1811.00461 [astro-ph.SR]

Cranmer SR, Gibson SE, Riley P (2017) Origins of the ambient solar wind: implications for space weather. Space Sci Rev 212(3-4):1345-1384. https://doi.org/10.1007/s11214-017-0416-y. arXiv: 1708.07169 [astro-ph.SR]

Cremades H, Bothmer V (2004) On the three-dimensional configuration of coronal mass ejections. Astron Astrophys 422:307-322. https://doi.org/10.1051/0004-6361:20035776

Cremades H, Iglesias FA, St Cyr OC, Xie H, Kaiser ML, Gopalswamy N (2015) Low-frequency Type-II radio detections and coronagraph data employed to describe and forecast the propagation of 71 CMEs/shocks. Sol Phys 290(9):2455-2478. https://doi.org/10.1007/s11207-015-0776-y. arXiv: 1505.01730 [astro-ph.SR]

Dagnew FK, Gopalswamy N, Tessema SB (2020a) A comparison of CME expansion speeds between solar cycles 23 and 24. J Phys Conf Ser 1620:012003. https://doi.org/10.1088/1742-6596/1620/1/ 012003

Dagnew FK, Gopalswamy N, Tessema SB, Akiyama S, Yashiro S, Tesfu TY (2020b) Intercycle and intracycle variation of halo CME rate obtained from SOHO/LASCO observations. Astrophys J 903(2):118. https://doi.org/10.3847/1538-4357/abb887

Dal Lago A, Schwenn R, Gonzalez WD (2003) Relation between the radial speed and the expansion speed of coronal mass ejections. Adv Space Res 32(12):2637-2640. https://doi.org/10.1016/j.asr. 2003.03.012

Dasso S, Nakwacki MS, Démoulin P, Mandrini CH (2007) Progressive transformation of a flux rope to an ICME. Comparative analysis using the direct and fitted expansion methods. Sol Phys 244(1-2):115-137. https://doi.org/10.1007/s11207-007-9034-2. arXiv:0706.2889 [astro-ph]

Davies JA, Harrison RA, Rouillard AP, Sheeley NR, Perry CH, Bewsher D, Davis CJ, Eyles CJ, Crothers SR, Brown DS (2009) A synoptic view of solar transient evolution in the inner heliosphere using the heliospheric imagers on STEREO. Geophys Res Lett 36(2):L02102. https://doi.org/10.1029/ 2008GL036182

Davis CJ, Kennedy J, Davies JA (2010) Assessing the accuracy of cme speed and trajectory estimates from STEREO observations through a comparison of independent methods. Sol Phys 263(1-2):209-222. https://doi.org/10.1007/s11207-010-9535-2

DeForest CE, Howard TA, McComas DJ (2013) Tracking coronal features from the low corona to Earth: A quantitative analysis of the 2008 December 12 coronal mass ejection. Astrophys J 769:43. https:// doi.org/10.1088/0004-637X/769/1/43

DeForest CE, Howard RA, Velli M, Viall N, Vourlidas A (2018) The highly structured outer solar corona. Astrophys J 862(1):18. https://doi.org/10.3847/1538-4357/aac8e3

Delaboudinière JP, Artzner GE, Brunaud J, Gabriel AH, Hochedez JF, Millier F, Song XY, Au B, Dere KP, Howard RA, Kreplin R, Michels DJ, Moses JD, Defise JM, Jamar C, Rochus P, Chauvineau JP, Marioge JP, Catura RC, Lemen JR, Shing L, Stern RA, Gurman JB, Neupert WM, Maucherat A, Clette F, Cugnon P, van Dessel EL (1995) EIT: Extreme-ultraviolet Imaging Telescope for the SOHO mission. Sol Phys 162(1-2):291-312. https://doi.org/10.1007/BF00733432

Démoulin P (2008) A review of the quantitative links between CMEs and magnetic clouds. Ann Geophys 26(10):3113-3125. https://doi.org/10.5194/angeo-26-3113-2008

Desai M, Giacalone J (2016) Large gradual solar energetic particle events. Living Rev Sol Phys 13:3. https://doi.org/10.1007/s41116-016-0002-5

Desai MI, Mason GM, Gold RE, Krimigis SM, Cohen CMS, Mewaldt RA, Mazur JE, Dwyer JR (2006) Heavy-ion elemental abundances in large solar energetic particle events and their implications for the seed population. Astrophys J 649(1):470-489. https://doi.org/10.1086/505649

D'Huys E, Seaton DB, Poedts S, Berghmans D (2014) Observational characteristics of coronal mass ejections without low-coronal signatures. Astrophys J 795:49. https://doi.org/10.1088/0004-637X/ $795 / 1 / 49.1409 .1422$

Dierckxsens M, Tziotziou K, Dalla S, Patsou I, Marsh MS, Crosby NB, Malandraki O, Tsiropoula G (2015) Relationship between solar energetic particles and properties of flares and CMEs: statistical analysis of solar cycle 23 events. Sol Phys 290(3):841-874. https://doi.org/10.1007/s11207-0140641-4. arXiv:1410.6070 [astro-ph.SR] 
Dissauer K, Veronig AM, Temmer M, Podladchikova T, Vanninathan K (2018) Statistics of coronal dimmings associated with coronal mass ejections. I. Characteristic dimming properties and flare association. Astrophys J 863(2):169. https://doi.org/10.3847/1538-4357/aad3c6. arXiv:1807.05056 [astro-ph.SR]

Dissauer K, Veronig AM, Temmer M, Podladchikova T (2019) Statistics of coronal dimmings associated with coronal mass ejections. II. Relationship between coronal dimmings and their associated CMEs. Astrophys J 874(2):123. https://doi.org/10.3847/1538-4357/ab0962. arXiv:1810.01589 [astroph.SR]

Domingo V, Fleck B, Poland AI (1995) The SOHO mission: an overview. Sol Phys 162(1-2):1-37. https://doi.org/10.1007/BF00733425

Dresing N, Gómez-Herrero R, Heber B, Hidalgo MA, Klassen A, Temmer M, Veronig A (2016) Injection of solar energetic particles into both loop legs of a magnetic cloud. Astron Astrophys 586:A55. https://doi.org/10.1051/0004-6361/201527347. arXiv:1601.00491 [physics.space-ph]

Dresing N, Gómez-Herrero R, Heber B, Klassen A, Temmer M, Veronig A (2018) Long-lasting injection of solar energetic electrons into the heliosphere. Astron Astrophys 613:A21. https://doi.org/10.1051/ 0004-6361/201731573. arXiv:1802.04722 [physics.space-ph]

Dumbović M, Devos A, Vršnak B, Sudar D, Rodriguez L, Ruždjak D, Leer K, Vennerstrøm S, Veronig A (2015) Geoeffectiveness of coronal mass ejections in the SOHO era. Sol Phys 290(2):579-612. https://doi.org/10.1007/s11207-014-0613-8. arXiv:1410.3303 [astro-ph.SR]

Dumbović M, Čalogović J, Vršnak B, Temmer M, Mays ML, Veronig A, Piantschitsch I (2018) The dragbased ensemble model (DBEM) for coronal mass ejection propagation. Astrophys J 854(2):12. https://doi.org/10.3847/1538-4357/aaaa66

Dumbović M, Guo J, Temmer M, Mays ML, Veronig A, Heinemann SG, Dissauer K, Hofmeister S, Halekas J, Möstl C, Amerstorfer T, Hinterreiter J, Banjac S, Herbst K, Wang Y, Holzknecht L, Leitner M, Wimmer-Schweingruber RF (2019) Unusual plasma and particle signatures at mars and STEREO_a related to CME-CME interaction. Astrophys J 880(1):18. https://doi.org/10.3847/ 1538-4357/ab27caarXiv:1906.02532 [astro-ph.SR]

Eastwood JP, Nakamura R, Turc L, Mejnertsen L, Hesse M (2017) The scientific foundations of forecasting magnetospheric space weather. Space Sci Rev 212(3-4):1221-1252. https://doi.org/10. 1007/s11214-017-0399-8

Emslie AG, Kucharek H, Dennis BR, Gopalswamy N, Holman GD, Share GH, Vourlidas A, Forbes TG, Gallagher PT, Mason GM, Metcalf TR, Mewaldt RA, Murphy RJ, Schwartz RA, Zurbuchen TH (2004) Energy partition in two solar flare/CME events. J Geophys Res 109(A10):A10104. https:// doi.org/10.1029/2004JA010571

Emslie AG, Dennis BR, Shih AY, Chamberlin PC, Mewaldt RA, Moore CS, Share GH, Vourlidas A, Welsch BT (2012) Global energetics of thirty-eight large solar eruptive events. Astrophys J 759(1):71. https://doi.org/10.1088/0004-637X/759/1/71. arXiv:1209.2654 [astro-ph.SR]

Eyles CJ, Simnett GM, Cooke MP, Jackson BV, Buffington A, Hick PP, Waltham NR, King JM, Anderson PA, Holladay PE (2003) The solar mass ejection imager (SMEI). Sol Phys 217(2):319-347. https://doi.org/10.1023/B:SOLA.0000006903.75671.49

Eyles CJ, Harrison RA, Davis CJ, Waltham NR, Shaughnessy BM, Mapson-Menard HCA, Bewsher D, Crothers SR, Davies JA, Simnett GM, Howard RA, Moses JD, Newmark JS, Socker DG, Halain JP, Defise JM, Mazy E, Rochus P (2009) The heliospheric imagers onboard the STEREO mission. Sol Phys 254(2):387-445. https://doi.org/10.1007/s11207-008-9299-0

Fan Y, Gibson SE (2007) Onset of coronal mass ejections due to loss of confinement of coronal flux ropes. Astrophys J 668(2):1232-1245. https://doi.org/10.1086/521335

Farrugia C, Berdichevsky D (2004) Evolutionary signatures in complex ejecta and their driven shocks. Ann Geophys 22(10):3679-3698. https://doi.org/10.5194/angeo-22-3679-2004

Farrugia CJ, Jordanova VK, Thomsen MF, Lu G, Cowley SWH, Ogilvie KW (2006) A two-ejecta event associated with a two-step geomagnetic storm. J Geophys Res 111(A11):A11104. https://doi.org/10. 1029/2006JA011893

Farrugia CJ, Berdichevsky DB, Möstl C, Galvin AB, Leitner M, Popecki MA, Simunac KDC, Opitz A, Lavraud B, Ogilvie KW, Veronig AM, Temmer M, Luhmann JG, Sauvaud JA (2011) Multiple, distant $\left(40^{\circ}\right)$ in situ observations of a magnetic cloud and a corotating interaction region complex. J Atmos Sol-Terr Phys 73(10):1254-1269. https://doi.org/10.1016/j.jastp.2010.09.011

Filippov B, Koutchmy S (2002) About the prominence heating mechanisms during its eruptive phase. Sol Phys 208(2):283-295. https://doi.org/10.1023/A:1020532607451 
Fletcher L, Dennis BR, Hudson HS, Krucker S, Phillips K, Veronig A, Battaglia M, Bone L, Caspi A, Chen Q, Gallagher P, Grigis PT, Ji H, Liu W, Milligan RO, Temmer M (2011) An observational overview of solar flares. Space Sci Rev 159(1-4):19-106. https://doi.org/10.1007/s11214-010-97018. arXiv:1109.5932 [astro-ph.SR]

Fokker AD (1963) Type IV solar radio emission. Space Sci Rev 2(1):70-90. https://doi.org/10.1007/ BF00174028

Forbes TG (2000) A review on the genesis of coronal mass ejections. J Geophys Res 105(A10):23153-23166. https://doi.org/10.1029/2000JA000005

Fox NJ, Velli MC, Bale SD, Decker R, Driesman A, Howard RA, Kasper JC, Kinnison J, Kusterer M, Lario D, Lockwood MK, McComas DJ, Raouafi NE, Szabo A (2016) The solar probe plus mission: humanity's first visit to our star. Space Sci Rev 204(1-4):7-48. https://doi.org/10.1007/s11214-0150211-6

Galvin AB, Kistler LM, Popecki MA, Farrugia CJ, Simunac KDC, Ellis L, Möbius E, Lee MA, Boehm M, Carroll J, Crawshaw A, Conti M, Demaine P, Ellis S, Gaidos JA, Googins J, Granoff M, Gustafson A, Heirtzler D, King B, Knauss U, Levasseur J, Longworth S, Singer K, Turco S, Vachon P, Vosbury M, Widholm M, Blush LM, Karrer R, Bochsler P, Daoudi H, Etter A, Fischer J, Jost J, Opitz A, Sigrist M, Wurz P, Klecker B, Ertl M, Seidenschwang E, Wimmer-Schweingruber RF, Koeten M, Thompson B, Steinfeld D (2008) The plasma and suprathermal ion composition (PLASTIC) investigation on the STEREO observatories. Space Sci Rev 136(1-4):437-486. https:// doi.org/10.1007/s11214-007-9296-X

Garton TM, Murray SA, Gallagher PT (2018) Expansion of high-speed solar wind streams from coronal holes through the inner heliosphere. Astrophys J Lett 869(1):L12. https://doi.org/10.3847/20418213/aaf39a. arXiv:1811.11605 [astro-ph.SR]

Georgoulis MK, Rust DM (2007) Quantitative forecasting of major solar flares. Astrophys J Lett 661(1):L109-L112. https://doi.org/10.1086/518718

Glesener L, Krucker S, Bain HM, Lin RP (2013) Observation of heating by flare-accelerated electrons in a solar coronal mass ejection. Astrophys J Lett 779(2):L29. https://doi.org/10.1088/2041-8205/779/ 2/L29

Gold T (1962) Magnetic storms. Space Sci Rev 1(1):100-114. https://doi.org/10.1007/BF00174637

Gombosi TI, van der Holst B, Manchester WB, Sokolov IV (2018) Extended MHD modeling of the steady solar corona and the solar wind. Living Rev Sol Phys 15:4. https://doi.org/10.1007/s41116018-0014-4. arXiv:1807.00417 [astro-ph.SR]

Gómez-Herrero R, Malandraki O, Dresing N, Kilpua E, Heber B, Klassen A, Müller-Mellin R, WimmerSchweingruber RF (2011) Spatial and temporal variations of CIRs: multi-point observations by STEREO. J Atmos Sol-Terr Phys 73(4):551-565. https://doi.org/10.1016/j.jastp.2010.11.017

Gómez-Herrero R, Dresing N, Klassen A, Heber B, Temmer M, Veronig A, Bučík R, Hidalgo MA, Carcaboso F, Blanco JJ, Lario D (2017) Sunward-propagating solar energetic electrons inside multiple interplanetary flux ropes. Astrophys J 840(2):85. https://doi.org/10.3847/1538-4357/aa6c5c

Good SW, Forsyth RJ, Eastwood JP, Möstl C (2018) Correlation of ICME magnetic fields at radially aligned spacecraft. Sol Phys 293(3):52. https://doi.org/10.1007/s11207-018-1264-y. arXiv:1802. 04004 [physics.space-ph]

Gopalswamy N (2016) History and development of coronal mass ejections as a key player in solar terrestrial relationship. Geosci Lett 3:8. https://doi.org/10.1186/s40562-016-0039-2. arXiv:1602. 03665 [astro-ph.SR]

Gopalswamy N (2018) Extreme solar eruptions and their space weather consequences. In: Buzulukova N (ed) Extreme events in geospace: origins, predictability, and consequences. Elsevier, Amsterdam, pp 37-63. https://doi.org/10.1016/B978-0-12-812700-1.00002-9

Gopalswamy N, Thompson BJ (2000) Early life of coronal mass ejections. J Atmos Sol-Terr Phys 62(16):1457-1469. https://doi.org/10.1016/S1364-6826(00)00079-1

Gopalswamy N, Lara A, Lepping RP, Kaiser ML, Berdichevsky D, St Cyr OC (2000) Interplanetary acceleration of coronal mass ejections. Geophys Res Lett 27(2):145-148. https://doi.org/10.1029/ 1999GL003639

Gopalswamy N, Lara A, Yashiro S, Kaiser ML, Howard RA (2001a) Predicting the 1-AU arrival times of coronal mass ejections. J Geophys Res 106(A12):29207-29218. https://doi.org/10.1029/ 2001JA000177

Gopalswamy N, Yashiro S, Kaiser ML, Howard RA, Bougeret JL (2001b) Characteristics of coronal mass ejections associated with long-wavelength type II radio bursts. J Geophys Res 106(A12):29219-29230. https://doi.org/10.1029/2001JA000234 
Gopalswamy N, Yashiro S, Michałek G, Kaiser ML, Howard RA, Reames DV, Leske R, von Rosenvinge $\mathrm{T}$ (2002) Interacting coronal mass ejections and solar energetic particles. Astrophys $\mathbf{J}$ Lett 572(1):L103-L107. https://doi.org/10.1086/341601

Gopalswamy N, Yashiro S, Krucker S, Stenborg G, Howard RA (2004) Intensity variation of large solar energetic particle events associated with coronal mass ejections. J Geophys Res 109(A12):A12105. https://doi.org/10.1029/2004JA010602

Gopalswamy N, Yashiro S, Liu Y, Michalek G, Vourlidas A, Kaiser ML, Howard RA (2005a) Coronal mass ejections and other extreme characteristics of the 2003 October-November solar eruptions. J Geophys Res 110(A9):A09S15. https://doi.org/10.1029/2004JA010958

Gopalswamy N, Yashiro S, Michalek G, Xie H, Lepping RP, Howard RA (2005b) Solar source of the largest geomagnetic storm of cycle 23. Geophys Res Lett 32(12):L12S09. https://doi.org/10.1029/ 2004GL021639

Gopalswamy N, Akiyama S, Yashiro S, Michalek G, Lepping RP (2008a) Solar sources and geospace consequences of interplanetary magnetic clouds observed during solar cycle 23. J Atmos Sol-Terr Phys 70(2-4):245-253. https://doi.org/10.1016/j.jastp.2007.08.070

Gopalswamy N, Yashiro S, Akiyama S, Mäkelä P, Xie H, Kaiser ML, Howard RA, Bougeret JL (2008b) Coronal mass ejections, type II radio bursts, and solar energetic particle events in the SOHO era. Ann Geophys 26(10):3033-3047. https://doi.org/10.5194/angeo-26-3033-2008

Gopalswamy N, Akiyama S, Yashiro S (2009) Major solar flares without coronal mass ejections. In: Gopalswamy N, Webb DF (eds) Universal heliophysical processes, vol 257. pp 283-286. https://doi. org/10.1017/S174392130902941X

Gopalswamy N, Davila JM, St Cyr OC, Sittler EC, Auchère F, Duvall TL, Hoeksema JT, Maksimovic M, MacDowall RJ, Szabo A, Collier MR (2011) Earth-affecting solar causes observatory (EASCO): a potential international living with a star mission from Sun-Earth L5. J Atmos Sol-Terr Phys 73(5-6):658-663. https://doi.org/10.1016/j.jastp.2011.01.013

Gopalswamy N, Nitta N, Akiyama S, Mäkelä P, Yashiro S (2012) Coronal magnetic field measurement from EUV images made by the solar dynamics observatory. Astrophys J 744(1):72. https://doi.org/ 10.1088/0004-637X/744/1/72. arXiv:1109.2925 [astro-ph.SR]

Gopalswamy N, Mäkelä P, Akiyama S, Xie H, Yashiro S, Reinard AA (2013a) The solar connection of enhanced heavy ion charge states in the interplanetary medium: implications for the flux-rope structure of CMEs. Sol Phys 284(1):17-46. https://doi.org/10.1007/s11207-012-0215-2. arXiv:1212. 0900 [astro-ph.SR]

Gopalswamy N, Xie H, Akiyama S, Yashiro S, Usoskin IG, Davila JM (2013b) The first ground level enhancement event of solar cycle 24: direct observation of shock formation and particle release heights. Astrophys J Lett 765(2):L30. https://doi.org/10.1088/2041-8205/765/2/L30. arXiv:1302. 1474 [astro-ph.SR]

Gopalswamy N, Akiyama S, Yashiro S, Xie H, Mäkelä P, Michalek G (2014) Anomalous expansion of coronal mass ejections during solar cycle 24 and its space weather implications. Geophys Res Lett 41(8):2673-2680. https://doi.org/10.1002/2014GL059858. arXiv:1404.0252 [astro-ph.SR]

Gopalswamy N, Yashiro S, Xie H, Akiyama S, Mäkelä P (2015) Properties and geoeffectiveness of magnetic clouds during solar cycles 23 and 24. J Geophys Res 120(11):9221-9245. https://doi.org/ 10.1002/2015JA021446. arXiv:1510.00906 [astro-ph.SR]

Gopalswamy N, Yashiro S, Thakur N, Mäkelä P, Xie H, Akiyama S (2016) The 2012 July 23 backside eruption: an extreme energetic particle event? Astrophys J 833(2):216. https://doi.org/10.3847/15384357/833/2/216arXiv:1610.05790 [astro-ph.SR]

Gopalswamy N, Mäkelä P, Yashiro S, Thakur N, Akiyama S, Xie H (2017a) A hierarchical relationship between the fluence spectra and CME kinematics in large solar energetic particle events: a radio perspective. J Phys Conf Ser 900:012009. https://doi.org/10.1088/1742-6596/900/1/012009. arXiv: 1707.00209 [astro-ph.SR]

Gopalswamy N, Yashiro S, Akiyama S, Xie H (2017b) Estimation of reconnection flux using posteruption arcades and its relevance to magnetic clouds at 1 AU. Sol Phys 292(4):65. https://doi.org/ 10.1007/s11207-017-1080-9. arXiv:1701.01943 [astro-ph.SR]

Gopalswamy N, Mäkelä P, Yashiro S, Lara A, Xie H, Akiyama S, MacDowall RJ (2018a) Interplanetary Type II radio bursts from Wind/WAVES and sustained gamma-ray emission from Fermi/LAT: evidence for shock source. Astrophys J Lett 868(2):L19. https://doi.org/10.3847/2041-8213/aaef36

Gopalswamy N, Yashiro S, Mäkelä P, Xie H, Akiyama S, Monstein C (2018b) Extreme kinematics of the 2017 September 10 solar eruption and the spectral characteristics of the associated energetic 
particles. Astrophys J Lett 863(2):L39. https://doi.org/10.3847/2041-8213/aad86c. arXiv:1807. 09906 [astro-ph.SR]

Grandin M, Aikio AT, Kozlovsky A (2019) Properties and geoeffectiveness of solar wind high-speed streams and stream interaction regions during solar cycles 23 and 24. J Geophys Res 124(6):3871-3892. https://doi.org/10.1029/2018JA026396. arXiv:2006.06302 [physics.space-ph]

Green LM, Török T, Vršnak B, Manchester W, Veronig A (2018) The origin, early evolution and predictability of solar eruptions. Space Sci Rev 214(1):46. https://doi.org/10.1007/s11214-0170462-5. arXiv:1801.04608 [astro-ph.SR]

Gressl C, Veronig AM, Temmer M, Odstrčil D, Linker JA, Mikić Z, Riley P (2014) Comparative study of MHD modeling of the background solar wind. Sol Phys 289(5):1783-1801. https://doi.org/10.1007/ s11207-013-0421-6. arXiv:1312.1220 [astro-ph.SR]

Gui B, Shen C, Wang Y, Ye P, Liu J, Wang S, Zhao X (2011) Quantitative analysis of CME deflections in the corona. Sol Phys 271(1-2):111-139. https://doi.org/10.1007/s11207-011-9791-9. arXiv:1105. 3382 [astro-ph.SR]

Gulisano AM, Démoulin P, Dasso S, Rodriguez L (2012) Expansion of magnetic clouds in the outer heliosphere. Astron Astrophys 543:A107. https://doi.org/10.1051/0004-6361/201118748. arXiv: 1206.1532 [physics.space-ph]

Guo J, Dumbović M, Wimmer-Schweingruber RF, Temmer M, Lohf H, Wang Y, Veronig A, Hassler DM, Mays LM, Zeitlin C, Ehresmann B, Witasse O, Freiherr von Forstner JL, Heber B, Holmström M, Posner A (2018) Modeling the evolution and propagation of 10 September 2017 CMEs and SEPs arriving at mars constrained by remote sensing and in situ measurement. Space Weather. https://doi. org/10.1029/2018SW001973

Guo Y, Ding MD, Schmieder B, Li H, Török T, Wiegelmann T (2010) Driving mechanism and onset condition of a confined eruption. Astrophys J Lett 725(1):L38-L42. https://doi.org/10.1088/20418205/725/1/L38

Harrison RA, Davis CJ, Eyles CJ, Bewsher D, Crothers SR, Davies JA, Howard RA, Moses DJ, Socker DG, Newmark JS, Halain JP, Defise JM, Mazy E, Rochus P, Webb DF, Simnett GM (2008) First imaging of coronal mass ejections in the heliosphere viewed from outside the Sun-Earth line. Sol Phys 247(1):171-193. https://doi.org/10.1007/s11207-007-9083-6

Harrison RA, Davies JA, Möstl C, Liu Y, Temmer M, Bisi MM, Eastwood JP, de Koning CA, Nitta N, Rollett T, Farrugia CJ, Forsyth RJ, Jackson BV, Jensen EA, Kilpua EKJ, Odstrcil D, Webb DF (2012) An analysis of the origin and propagation of the multiple coronal mass ejections of 2010 August 1. Astrophys J 750(1):45. https://doi.org/10.1088/0004-637X/750/1/45

Harvey KL, Recely F (2002) Polar coronal holes during cycles 22 and 23. Sol Phys 211(1):31-52. https:// doi.org/10.1023/A:1022469023581

Hassler DM, Zeitlin C, Ehresmann B, Wimmer-Schweingruber RF, Guo J, Matthiä D, Rafkin S, Berger T, Reitz G (2018) Space weather on the surface of Mars: impact of the September 2017 events. Space Weather 16(11):1702-1708. https://doi.org/10.1029/2018SW001959

Hassler DM, Newmark J, Gibson S, Harra L, Appourchaux T, Auchere F, Berghmans D, Colaninno R, Fineschi S, Gizon L, Gosain S, Hoeksema T, Kintziger C, Linker J, Rochus P, Schou J, Viall N, West M, Woods T, Wuelser JP (2020) The Solaris solar polar mission. In: EGU General Assembly 2020. Copernicus, p 17703. https://doi.org/10.5194/egusphere-egu2020-17703

Hathaway DH (2010) The solar cycle. Living Rev Sol Phys 7:1. https://doi.org/10.12942/lrsp-2010-1

Hayakawa H, Ribeiro P, Vaquero JM, Gallego MC, Knipp DJ, Mekhaldi F, Bhaskar A, Oliveira DM, Notsu Y, Carrasco VMS, Caccavari A, Veenadhari B, Mukherjee S, Ebihara Y (2020) The extreme space weather event in 1903 October/November: an outburst from the quiet Sun. Astrophys J Lett 897(1):L10. https://doi.org/10.3847/2041-8213/ab6a18. arXiv:2001.04575 [astro-ph.SR]

Heinemann SG, Temmer M, Farrugia CJ, Dissauer K, Kay C, Wiegelmann T, Dumbović M, Veronig AM, Podladchikova T, Hofmeister SJ, Lugaz N, Carcaboso F (2019) CME-HSS interaction and characteristics tracked from Sun to Earth. Sol Phys 294(9):121. https://doi.org/10.1007/s11207-0191515-6. arXiv:1908.10161 [astro-ph.SR]

Heinemann SG, Temmer M, Heinemann N, Dissauer K, Samara E, Jerčić V, Hofmeister SJ, Veronig AM (2019) Statistical analysis and catalog of non-polar coronal holes covering the SDO-era using CATCH. Sol Phys. https://doi.org/10.1007/s11207-019-1539-y

Heinemann SG, Jerčić V, Temmer M, Hofmeister SJ, Dumbović M, Vennerstrom S, Verbanac G, Veronig AM (2020) A statistical study of the long-term evolution of coronal hole properties as observed by SDO. Astron Astrophys 638:A68. https://doi.org/10.1051/0004-6361/202037613. arXiv:1907.02795 [astro-ph.SR] 
Hess P, Zhang J (2017) A study of the Earth-affecting CMEs of solar cycle 24. Sol Phys 292(6):80. https://doi.org/10.1007/s11207-017-1099-y

Hirayama T (1974) Theoretical model of flares and prominences. I: Evaporating flare model. Sol Phys 34(2):323-338. https://doi.org/10.1007/BF00153671

Hofmeister SJ, Veronig A, Reiss MA, Temmer M, Vennerstrom S, Vršnak B, Heber B (2017) Characteristics of low-latitude coronal holes near the maximum of solar cycle 24. Astrophys J. https://doi.org/10.3847/1538-4357/835/2/268

Hofmeister SJ, Veronig A, Temmer M, Vennerstrom S, Heber B, Vršnak B (2018) The dependence of the peak velocity of high-speed solar wind streams as measured in the ecliptic by ACE and the STEREO satellites on the area and co-latitude of their solar source coronal holes. J Geophys Res 123(3):1738-1753. https://doi.org/10.1002/2017JA024586. arXiv:1804.09579 [astro-ph.SR]

Holman GD, Pesses ME (1983) Solar type II radio emission and the shock drift acceleration of electrons. Astrophys J 267:837-843. https://doi.org/10.1086/160918

Howard RA (2006) A historical perspective on coronal mass ejections. In: Gopalswamy N, Mewaldt R, Torsti J (eds) Solar eruptions and energetic particles. Geophysical monograph series, vol 165. American Geophysical Union, Washington, DC, p 7. https://doi.org/10.1029/165GM03

Howard RA, Moses JD, Vourlidas A, Newmark JS, Socker DG, Plunkett SP, Korendyke CM, Cook JW, Hurley A, Davila JM, Thompson WT, St Cyr OC, Mentzell E, Mehalick K, Lemen JR, Wuelser JP, Duncan DW, Tarbell TD, Wolfson CJ, Moore A, Harrison RA, Waltham NR, Lang J, Davis CJ, Eyles CJ, Mapson-Menard H, Simnett GM, Halain JP, Defise JM, Mazy E, Rochus P, Mercier R, Ravet MF, Delmotte F, Auchere F, Delaboudiniere JP, Bothmer V, Deutsch W, Wang D, Rich N, Cooper S, Stephens V, Maahs G, Baugh R, McMullin D, Carter T (2008) Sun Earth Connection Coronal and Heliospheric Investigation (SECCHI). Space Sci Rev 136(1-4):67-115. https://doi.org/ 10.1007/s11214-008-9341-4

Howard RA, Vourlidas A, Bothmer V, Colaninno RC, DeForest CE, Gallagher B, Hall JR, Hess P, Higginson AK, Korendyke CM, Kouloumvakos A, Lamy PL, Liewer PC, Linker J, Linton M, Penteado P, Plunkett SP, Poirier N, Raouafi NE, Rich N, Rochus P, Rouillard AP, Socker DG, Stenborg G, Thernisien AF, Viall NM (2019) Near-Sun observations of an F-corona decrease and K-corona fine structure. Nature 576(7786):232-236. https://doi.org/10.1038/s41586-019-1807-x

Howard RA, Vourlidas A, Colaninno RC, Korendyke CM, Plunkett SP, Carter MT, Wang D, Rich N, Lynch S, Thurn A, Socker DG, Thernisien AF, Chua D, Linton MG, Koss S, Tun-Beltran S, Dennison H, Stenborg G, McMullin DR, Hunt T, Baugh R, Clifford G, Keller D, Janesick JR, Tower J, Grygon M, Farkas R, Hagood R, Eisenhauer K, Uhl A, Yerushalmi S, Smith L, Liewer PC, Velli MC, Linker J, Bothmer V, Rochus P, Halain JP, Lamy PL, Auchère F, Harrison RA, Rouillard A, Patsourakos S, St Cyr OC, Gilbert H, Maldonado H, Mariano C, Cerullo J (2020) The Solar Orbiter Heliospheric Imager (SoloHI). Astron Astrophys 642:A13. https://doi.org/10.1051/0004-6361/ 201935202

Howard TA, Harrison RA (2013) Stealth coronal mass ejections: a perspective. Sol Phys 285(1-2):269-280. https://doi.org/10.1007/s11207-012-0217-0

Howard TA, Tappin SJ (2008) Three-dimensional reconstruction of two solar coronal mass ejections using the STEREO spacecraft. Sol Phys 252(2):373-383. https://doi.org/10.1007/s11207-008-92620

Howard TA, DeForest CE, Schneck UG, Alden CR (2017) Challenging some contemporary views of coronal mass ejections. II. The case for absent filaments. Astrophys J 834(1):86. https://doi.org/10. $3847 / 1538-4357 / 834 / 1 / 86$

Hudson HS (2011) Global properties of solar flares. Space Sci Rev 158(1):5-41. https://doi.org/10.1007/ s11214-010-9721-4arXiv:1108.3490 [astro-ph.SR]

Hudson HS, Acton LW, Freeland SL (1996) A long-duration solar flare with mass ejection and global consequences. Astrophys J 470:629. https://doi.org/10.1086/177894

Hundhausen AJ (1993) Sizes and locations of coronal mass ejections: SMM observations from 1980 and 1984-1989. J Geophys Res 98(A8):13177-13200. https://doi.org/10.1029/93JA00157

Isavnin A, Vourlidas A, Kilpua EKJ (2014) Three-dimensional evolution of flux-rope CMEs and its relation to the local orientation of the heliospheric current sheet. Sol Phys 289(6):2141-2156. https://doi.org/10.1007/s11207-013-0468-4. arXiv:1312.0458 [physics.space-ph]

Jang S, Moon YJ, Kim RS, Kim S, Lee JO (2017) Two distinct types of CME-flare relationships based on SOHO and STEREO observations. Astrophys J 845(2):169. https://doi.org/10.3847/1538-4357/ aa82b4 
Janvier M, Winslow RM, Good S, Bonhomme E, Démoulin P, Dasso S, Möstl C, Lugaz N, Amerstorfer T, Soubrié E, Boakes PD (2019) Generic magnetic field intensity profiles of interplanetary coronal mass ejections at Mercury, Venus, and Earth from superposed epoch analyses. J Geophys Res 124(2):812-836. https://doi.org/10.1029/2018JA025949. arXiv:1901.09921 [physics.space-ph]

Jian L, Russell CT, Luhmann JG, Skoug RM (2006) Properties of stream interactions at one AU during 1995-2004. Sol Phys 239(1-2):337-392. https://doi.org/10.1007/s11207-006-0132-3

Jian LK, Russell CT, Luhmann JG, Galvin AB, MacNeice PJ (2009) Multi-spacecraft observations: stream interactions and associated structures. Sol Phys 259(1-2):345-360. https://doi.org/10.1007/ s11207-009-9445-3

Jian LK, Russell CT, Luhmann JG (2011) Comparing solar minimum 23/24 with historical solar wind records at 1 AU. Sol Phys 274(1-2):321-344. https://doi.org/10.1007/s11207-011-9737-2

Jian LK, Russell CT, Luhmann JG, Galvin AB (2018) STEREO observations of interplanetary coronal mass ejections in 2007-2016. Astrophys J 855(2):114. https://doi.org/10.3847/1538-4357/aab189

Jian LK, Luhmann JG, Russell CT, Galvin AB (2019) Solar TErrestrial RElations Observatory (STEREO) observations of stream interaction regions in 2007-2016: relationship with heliospheric current sheets, solar cycle variations, and dual observations. Sol Phys 294(3):31. https://doi.org/10. 1007/s11207-019-1416-8

Kahler SW, Ling AG (2018) Relating solar energetic particle event fluences to peak intensities. Sol Phys 293(2):30. https://doi.org/10.1007/s11207-018-1249-x

Kahler SW, Vourlidas A (2005) Fast coronal mass ejection environments and the production of solar energetic particle events. J Geophys Res 110(A12):A12S01. https://doi.org/10.1029/2005JA011073

Kahler SW, Vourlidas A (2013) A comparison of the intensities and energies of gradual solar energetic particle events with the dynamical properties of associated coronal mass ejections. Astrophys $\mathrm{J}$ 769(2):143. https://doi.org/10.1088/0004-637X/769/2/143

Kahler SW, Cliver EW, Ling AG (2007) Validating the proton prediction system (PPS). J Atmos Sol-Terr Phys 69(1-2):43-49. https://doi.org/10.1016/j.jastp.2006.06.009

Kahler SW, Ling AG, Gopalswamy N (2019) Are solar energetic particle events and type II bursts associated with fast and narrow coronal mass ejections? Sol Phys 294(9):134. https://doi.org/10. 1007/s11207-019-1518-3

Kaiser ML, Kucera TA, Davila JM, St Cyr OC, Guhathakurta M, Christian E (2008) The STEREO mission: an introduction. Space Sci Rev 136(1-4):5-16. https://doi.org/10.1007/s11214-007-9277-0

Kasper JC, Bale SD, Belcher JW, Berthomier M, Case AW, Chandran BDG, Curtis DW, Gallagher D, Gary SP, Golub L, Halekas JS, Ho GC, Horbury TS, Hu Q, Huang J, Klein KG, Korreck KE, Larson DE, Livi R, Maruca B, Lavraud B, Louarn P, Maksimovic M, Martinovic M, McGinnis D, Pogorelov NV, Richardson JD, Skoug RM, Steinberg JT, Stevens ML, Szabo A, Velli M, Whittlesey PL, Wright KH, Zank GP, MacDowall RJ, McComas DJ, McNutt RL, Pulupa M, Raouafi NE, Schwadron NA (2019) Alfvénic velocity spikes and rotational flows in the near-Sun solar wind. Nature 576(7786):228-231. https://doi.org/10.1038/s41586-019-1813-z

Kay C, Gopalswamy N (2017) Using the coronal evolution to successfully forward model CMEs' in situ magnetic profiles. J Geophys Res 122(12):11810-11834. https://doi.org/10.1002/2017JA024541. arXiv:1710.03825 [physics.space-ph]

Kay C, Opher M (2015) The heliocentric distance where the deflections and rotations of solar coronal mass ejections occur. Astrophys J Lett 811(2):L36. https://doi.org/10.1088/2041-8205/811/2/L36. arXiv:1509.04948 [astro-ph.SR]

Kazachenko MD, Lynch BJ, Welsch BT, Sun X (2017) A database of flare ribbon properties from the Solar Dynamics Observatory. I. Reconnection flux. Astrophys J 845(1):49. https://doi.org/10.3847/ 1538-4357/aa7ed6. arXiv:1704.05097 [astro-ph.SR]

Kienreich IW, Temmer M, Veronig AM (2009) STEREO quadrature observations of the threedimensional structure and driver of a global coronal wave. Astrophys J Lett 703(2):L118-L122. https://doi.org/10.1088/0004-637X/703/2/L118. arXiv:0908.3571 [astro-ph.SR]

Kilpua E, Koskinen HEJ, Pulkkinen TI (2017) Coronal mass ejections and their sheath regions in interplanetary space. Living Rev Sol Phys 14:5. https://doi.org/10.1007/s41116-017-0009-6

Kilpua EKJ, Jian LK, Li Y, Luhmann JG, Russell CT (2011) Multipoint ICME encounters: pre-STEREO and STEREO observations. J Atmos Sol-Terr Phys 73(10):1228-1241. https://doi.org/10.1016/j. jastp.2010.10.012

Kilpua EKJ, Hietala H, Turner DL, Koskinen HEJ, Pulkkinen TI, Rodriguez JV, Reeves GD, Claudepierre SG, Spence HE (2015) Unraveling the drivers of the storm time radiation belt response. Geophys Res Lett 42(9):3076-3084. https://doi.org/10.1002/2015GL063542 
Klein KL, Dalla S (2017) Acceleration and propagation of solar energetic particles. Space Sci Rev 212(3-4):1107-1136. https://doi.org/10.1007/s11214-017-0382-4. arXiv:1705.07274 [astro-ph.SR]

Klein KL, Trottet G, Klassen A (2010) Energetic particle acceleration and propagation in strong CMEless flares. Sol Phys 263(1-2):185-208. https://doi.org/10.1007/s11207-010-9540-5

Kopp RA, Pneuman GW (1976) Magnetic reconnection in the corona and the loop prominence phenomenon. Sol Phys 50(1):85-98. https://doi.org/10.1007/BF00206193

Kouloumvakos A, Rouillard AP, Wu Y, Vainio R, Vourlidas A, Plotnikov I, Afanasiev A, Önel H (2019) Connecting the properties of coronal shock waves with those of solar energetic particles. Astrophys J 876(1):80. https://doi.org/10.3847/1538-4357/ab15d7

Kozarev KA, Korreck KE, Lobzin VV, Weber MA, Schwadron NA (2011) Off-limb solar coronal wavefronts from SDO/AIA extreme-ultraviolet observations-implications for particle production. Astrophys J Lett 733(2):L25. https://doi.org/10.1088/2041-8205/733/2/L25. arXiv:1406.2372 [astroph.SR]

Kozarev KA, Davey A, Kendrick A, Hammer M, Keith C (2017) The coronal analysis of shocks and waves (CASHeW) framework. J Space Weather Space Clim 7:A32. https://doi.org/10.1051/swsc/ 2017028. arXiv:1710.05302 [astro-ph.SR]

Künzel H (1960) Die Flare-Häufigkeit in Fleckengruppen unterschiedlicher Klasse und magnetischer Struktur. Astron Nachr 285(5):271. https://doi.org/10.1002/asna.19592850516

Kwon RY, Vourlidas A (2017) Investigating the wave nature of the outer envelope of halo coronal mass ejections. Astrophys J 836(2):246. https://doi.org/10.3847/1538-4357/aa5b92

Kwon RY, Vourlidas A (2018) The density compression ratio of shock fronts associated with coronal mass ejections. J Space Weather Space Clim 8:A08. https://doi.org/10.1051/swsc/2017045. arXiv: 1801.04355 [astro-ph.SR]

Kwon RY, Zhang J, Vourlidas A (2015) Are halo-like solar coronal mass ejections merely a matter of geometric projection effects? Astrophys J Lett 799(2):L29. https://doi.org/10.1088/2041-8205/799/ 2/L29

Lamy PL, Floyd O, Boclet B, Wojak J, Gilardy H, Barlyaeva T (2019) Coronal mass ejections over solar cycles 23 and 24. Space Sci Rev 215(5):39. https://doi.org/10.1007/s11214-019-0605-y

Lang M, Owens MJ (2019) A variational approach to data assimilation in the solar wind. Space Weather 17(1):59-83. https://doi.org/10.1029/2018SW001857. arXiv:1806.07306 [physics.space-ph]

Lario D, Karelitz A (2014) Influence of interplanetary coronal mass ejections on the peak intensity of solar energetic particle events. J Geophys Res 119(6):4185-4209. https://doi.org/10.1002/ 2014JA019771

Laurenza M, Cliver EW, Hewitt J, Storini M, Ling AG, Balch CC, Kaiser ML (2009) A technique for short-term warning of solar energetic particle events based on flare location, flare size, and evidence of particle escape. Space Weather 7(4):S04008. https://doi.org/10.1029/2007SW000379

Lavraud B, Liu Y, Segura K, He J, Qin G, Temmer M, Vial JC, Xiong M, Davies JA, Rouillard AP, Pinto R, Auchère F, Harrison RA, Eyles C, Gan W, Lamy P, Xia L, Eastwood JP, Kong L, Wang J, Wimmer-Schweingruber RF, Zhang S, Zong Q, Soucek J, An J, Prech L, Zhang A, Rochus P, Bothmer V, Janvier M, Maksimovic M, Escoubet CP, Kilpua EKJ, Tappin J, Vainio R, Poedts S, Dunlop MW, Savani N, Gopalswamy N, Bale SD, Li G, Howard T, DeForest C, Webb D, Lugaz N, Fuselier SA, Dalmasse K, Tallineau J, Vranken D, Fernández JG (2016) A small mission concept to the Sun-Earth Lagrangian L5 point for innovative solar, heliospheric and space weather science. J Atmos Sol-Terr Phys. https://doi.org/10.1016/j.jastp.2016.06.004

Leblanc Y, Dulk GA, Bougeret JL (1998) Tracing the electron density from the corona to 1AU. Sol Phys 183(1):165-180. https://doi.org/10.1023/A:1005049730506

Lee CO, Arge CN, Odstrčil D, Millward G, Pizzo V, Quinn JM, Henney CJ (2013) Ensemble modeling of CME propagation. Sol Phys 285(1-2):349-368. https://doi.org/10.1007/s11207-012-9980-1

Lee JY, Raymond JC, Ko YK, Kim KS (2009) Three-dimensional structure and energy balance of a coronal mass ejection. Astrophys J 692(2):1271-1286. https://doi.org/10.1088/0004-637X/692/2/ 1271. arXiv:0810.4950 [astro-ph]

Leitner M, Farrugia CJ, Möstl C, Ogilvie KW, Galvin AB, Schwenn R, Biernat HK (2007) Consequences of the force-free model of magnetic clouds for their heliospheric evolution. J Geophys Res 112(A6):A06113. https://doi.org/10.1029/2006JA011940

Li G, Zank GP (2005) Mixed particle acceleration at CME-driven shocks and flares. Geophys Res Lett 32(2):L02101. https://doi.org/10.1029/2004GL021250

Liebenberg DH, Bessey RJ, Watson B (1975) Observed coronal temperatures at $1.37 R_{\odot}$ in the region of a helmet structure. Sol Phys 40(2):387-396. https://doi.org/10.1007/BF00162385 
Liewer PC, Dejong EM, Hall JR, Howard RA, Thompson WT, Thernisien A (2010) Determination of CME trajectories by stereoscopic analysis of STEREO/SECCHI data. In: Maksimovic M, Issautier K, Meyer-Vernet N, Moncuquet M, Pantellini F (eds) Twelfth international solar wind conference. AIP conference series, vol 1216. American Institute of Physics, pp 412-415. https://doi.org/10.1063/ 1.3395891

Lin J, Forbes TG (2000) Effects of reconnection on the coronal mass ejection process. J Geophys Res 105(A2):2375-2392. https://doi.org/10.1029/1999JA900477

Lin RP (2006) Effects of reconnection on the coronal mass ejection process. Hard X-rays/Gamma-rays. Space Sci Rev 124(1-4):233-248. https://doi.org/10.1007/s11214-006-9107-9

Linker JA, Caplan RM, Downs C, Riley P, Mikić Z, Lionello R, Henney CJ, Arge CN, Liu Y, DeRosa ML, Yeates A, Owens MJ (2017) The open flux problem. Astrophys J 848(1):70. https://doi.org/10. 3847/1538-4357/aa8a70. arXiv:1708.02342 [astro-ph.SR]

Liu W, Jin M, Downs C, Ofman L, Cheung MCM, Nitta NV (2018) A truly global extreme ultraviolet wave from the SOL2017-09-10 X8.2+ solar flare-coronal mass ejection. Astrophys J 864(2):L24. https://doi.org/10.3847/2041-8213/aad77b

Liu YD, Luhmann JG, Lugaz N, Möstl C, Davies JA, Bale SD, Lin RP (2013) On Sun-to-Earth propagation of coronal mass ejections. Astrophys J 769(1):45. https://doi.org/10.1088/0004-637X/ 769/1/45. arXiv:1304.3777 [astro-ph.SR]

Liu YD, Luhmann JG, Kajdič P, Kilpua EKJ, Lugaz N, Nitta NV, Möstl C, Lavraud B, Bale SD, Farrugia CJ, Galvin AB (2014) Observations of an extreme storm in interplanetary space caused by successive coronal mass ejections. Nat Commun 5:3481. https://doi.org/10.1038/ncomms4481. arXiv:1405.6088 [physics.space-ph]

Lockwood M (2013) Reconstruction and prediction of variations in the open solar magnetic flux and interplanetary conditions. Living Rev Sol Phys 10:4. https://doi.org/10.12942/lrsp-2013-4

Longo F, Omodei N, Digel S (2017) Fermi LAT detection of X-class solar flares of September 6, 2017. The Astronomer's Telegram 10720:1

Lugaz N (2010) Accuracy and limitations of fitting and stereoscopic methods to determine the direction of coronal mass ejections from heliospheric imagers observations. Sol Phys 267(2):411-429. https:// doi.org/10.1007/s11207-010-9654-9. arXiv:1010.1949 [astro-ph.SR]

Lugaz N, Farrugia CJ, Huang CL, Spence HE (2015) Extreme geomagnetic disturbances due to shocks within CMEs. Geophys Res Lett 42(12):4694-4701. https://doi.org/10.1002/2015GL064530

Lugaz N, Farrugia CJ, Winslow RM, Small CR, Manion T, Savani NP (2017a) Importance of CME radial expansion on the ability of slow CMEs to drive shocks. Astrophys J 848(2):75. https://doi.org/10. 3847/1538-4357/aa8ef9. arXiv:1707.06676 [physics.space-ph]

Lugaz N, Temmer M, Wang Y, Farrugia CJ (2017b) The interaction of successive coronal mass ejections: a review. Sol Phys 292(4):64. https://doi.org/10.1007/s11207-017-1091-6. arXiv:1612.02398 [physics.space-ph]

Lugaz N, Farrugia CJ, Winslow RM, Al-Haddad N, Galvin AB, Nieves-Chinchilla T, Lee CO, Janvier M (2018) On the spatial coherence of magnetic ejecta: measurements of coronal mass ejections by multiple spacecraft longitudinally separated by 0.01 au. Astrophys J Lett 864(1):L7. https://doi.org/ 10.3847/2041-8213/aad9f4. arXiv:1812.00911 [physics.space-ph]

Lugaz N, Salman TM, Winslow RM, Al-Haddad N, Farrugia CJ, Zhuang B, Galvin AB (2020) Inconsistencies between local and global measures of CME radial expansion as revealed by spacecraft conjunctions. Astrophys J 899(2):119. https://doi.org/10.3847/1538-4357/aba26b. arXiv: 2007.01699 [physics.space-ph]

Luhmann JG, Curtis DW, Schroeder P, McCauley J, Lin RP, Larson DE, Bale SD, Sauvaud JA, Aoustin C, Mewaldt RA, Cummings AC, Stone EC, Davis AJ, Cook WR, Kecman B, Wiedenbeck ME, von Rosenvinge T, Acuna MH, Reichenthal LS, Shuman S, Wortman KA, Reames DV, Mueller-Mellin R, Kunow H, Mason GM, Walpole P, Korth A, Sanderson TR, Russell CT, Gosling JT (2008) STEREO IMPACT investigation goals, measurements, and data products overview. Space Sci Rev 136(1-4):117-184. https://doi.org/10.1007/s11214-007-9170-x

Luhmann JG, Gopalswamy N, Jian LK, Lugaz N (2020) ICME evolution in the inner heliosphere. Sol Phys 295(4):61. https://doi.org/10.1007/s11207-020-01624-0

Ma S, Raymond JC, Golub L, Lin J, Chen H, Grigis P, Testa P, Long D (2011) Observations and interpretation of a low coronal shock wave observed in the EUV by the SDO/AIA. Astrophys J 738(2):160. https://doi.org/10.1088/0004-637X/738/2/160. arXiv:1106.6056 [astro-ph.SR] 
Mackay DH, Yeates AR (2012) The Sun's global photospheric and coronal magnetic fields: observations and models. Living Rev Sol Phys 9:6. https://doi.org/10.12942/lrsp-2012-6. arXiv:1211.6545 [astroph.SR]

MacQueen RM, Eddy JA, Gosling JT, Hildner E, Munro RH, Newkirk JGA, Poland AI, Ross CL (1974) The outer solar corona as observed from Skylab: preliminary results. Astrophys J Lett 187:L85. https://doi.org/10.1086/181402

Magdalenić J, Marqué C, Krupar V, Mierla M, Zhukov AN, Rodriguez L, Maksimović M, Cecconi B (2014) Tracking the CME-driven shock wave on 2012 March 5 and radio triangulation of associated radio emission. Astrophys J 791(2):115. https://doi.org/10.1088/0004-637X/791/2/115

Mäkelä P, Gopalswamy N, Yashiro S (2016) The radial speed-expansion speed relation for Earth-directed CMEs. Space Weather 14(5):368-378. https://doi.org/10.1002/2015SW001335

Malandraki OE, Crosby NB (2018) Solar energetic particles and space weather: science and applications. In: Malandraki OE, Crosby NB (eds) Solar particle radiation storms forecasting and analysis. Astrophysics and Space Science Library, vol 444. Springer, Cham, pp 1-26. https://doi.org/10.1007/ 978-3-319-60051-2_1

Maloney SA, Gallagher PT, McAteer RTJ (2009) Reconstructing the 3-D trajectories of CMEs in the inner heliosphere. Sol Phys 256(1-2):149-166. https://doi.org/10.1007/s11207-009-9364-3. arXiv: 0905.2153 [astro-ph.SR]

Manchester W, Kilpua EKJ, Liu YD, Lugaz N, Riley P, Török T, Vršnak B (2017) The physical processes of CME/ICME evolution. Space Sci Rev 212(3-4):1159-1219. https://doi.org/10.1007/s11214-0170394-0

Manchester WB, Kozyra JU, Lepri ST, Lavraud B (2014) Simulation of magnetic cloud erosion during propagation. J Geophys Res 119(7):5449-5464. https://doi.org/10.1002/2014JA019882

Mandrini CH, Nakwacki MS, Attrill G, van Driel-Gesztelyi L, Démoulin P, Dasso S, Elliott H (2007) Are CME-related dimmings always a simple signature of interplanetary magnetic cloud footpoints? Sol Phys 244(1-2):25-43. https://doi.org/10.1007/s11207-007-9020-8

Mann G, Klassen A (2005) Electron beams generated by shock waves in the solar corona. Astron Astrophys 441(1):319-326. https://doi.org/10.1051/0004-6361:20034396

Mann G, Jansen F, MacDowall RJ, Kaiser ML, Stone RG (1999) A heliospheric density model and type III radio bursts. Astron Astrophys 348:614-620

Maričić D, Vršnak B, Stanger AL, Veronig AM, Temmer M, Roša D (2007) Acceleration phase of coronal mass ejections: II. Synchronization of the energy release in the associated flare. Sol Phys 241(1):99-112. https://doi.org/10.1007/s11207-007-0291-x

Maričić D, Vršnak B, Dumbović M, Žic T, Roša D, Hržina D, Lulić S, Romštajn I, Bušić I, Salamon K, Temmer M, Rollett T, Veronig A, Bostanjyan N, Chilingarian A, Mailyan B, Arakelyan K, Hovhannisyan A, Mujić N (2014) Kinematics of interacting ICMEs and related forbush decrease: case study. Sol Phys 289(1):351-368. https://doi.org/10.1007/s11207-013-0314-8

Marubashi K, Lepping RP (2007) Long-duration magnetic clouds: a comparison of analyses using torusand cylinder-shaped flux rope models. Ann Geophys 25:2453-2477. https://doi.org/10.5194/angeo25-2453-2007

Mason GM, Cohen CMS, Cummings AC, Dwyer JR, Gold RE, Krimigis SM, Leske RA, Mazur JE, Mewaldt RA, Möbius E, Popecki M, Stone EC, von Rosenvinge TT, Wiedenbeck ME (1999) Particle acceleration and sources in the November 1997 solar energetic particle events. Geophys Res Lett 26(2):141-144. https://doi.org/10.1029/1998GL900235

Masson S, Démoulin P, Dasso S, Klein KL (2012) The interplanetary magnetic structure that guides solar relativistic particles. Astron Astrophys 538:A32. https://doi.org/10.1051/0004-6361/201118145. arXiv:1110.6811 [astro-ph.SR]

Mays ML, Taktakishvili A, Pulkkinen A, MacNeice PJ, Rastätter L, Odstrcil D, Jian LK, Richardson IG, LaSota JA, Zheng Y, Kuznetsova MM (2015) Ensemble modeling of CMEs using the WSAENLIL+cone model. Sol Phys 290(6):1775-1814. https://doi.org/10.1007/s11207-015-06921arXiv: 1504.04402 [astro-ph.SR]

McComas DJ, Barraclough BL, Funsten HO, Gosling JT, Santiago-Muñoz E, Skoug RM, Goldstein BE, Neugebauer M, Riley P, Balogh A (2000) Solar wind observations over Ulysses' first full polar orbit. J Geophys Res 105(A5):10419-10434. https://doi.org/10.1029/1999JA000383

McComas DJ, Angold N, Elliott HA, Livadiotis G, Schwadron NA, Skoug RM, Smith CW (2013) Weakest solar wind of the space age and the current "mini" solar maximum. Astrophys J 779(1):2. https://doi.org/10.1088/0004-637X/779/1/2 
McIntosh PS (1990) The classification of sunspot groups. Sol Phys 125(2):251-267. https://doi.org/10. 1007/BF00158405

Mewaldt RA (2006) Solar energetic particle composition, energy spectra, and space weather. Space Sci Rev 124(1-4):303-316. https://doi.org/10.1007/s11214-006-9091-0

Mewaldt RA, Looper MD, Cohen CMS, Haggerty DK, Labrador AW, Leske RA, Mason GM, Mazur JE, von Rosenvinge TT (2012) Energy spectra, composition, and other properties of ground-level events during solar cycle 23. Space Sci Rev 171(1-4):97-120. https://doi.org/10.1007/s11214-012-9884-2

Michalek G, Gopalswamy N, Lara A, Yashiro S (2006) Properties and geoeffectiveness of halo coronal mass ejections. Space Weather 4(10):S10003. https://doi.org/10.1029/2005SW000218. arXiv:0710. 4526 [astro-ph]

Michalek G, Gopalswamy N, Yashiro S (2009) Expansion speed of coronal mass ejections. Sol Phys 260(2):401-406. https://doi.org/10.1007/s11207-009-9464-0

Mierla M, Inhester B, Antunes A, Boursier Y, Byrne JP, Colaninno R, Davila J, de Koning CA, Gallagher PT, Gissot S, Howard RA, Howard TA, Kramar M, Lamy P, Liewer PC, Maloney S, Marqué C, McAteer RTJ, Moran T, Rodriguez L, Srivastava N, St Cyr OC, Stenborg G, Temmer M, Thernisien A, Vourlidas A, West MJ, Wood BE, Zhukov AN (2010) On the 3-D reconstruction of coronal mass ejections using coronagraph data. Ann Geophys 28(1):203-215. https://doi.org/10.5194/angeo-28203-2010

Mikić Z, Lee MA (2006) An introduction to theory and models of CMEs, shocks, and solar energetic particles. Space Sci Rev 123(1-3):57-80. https://doi.org/10.1007/s11214-006-9012-2

Miroshnichenko LI (2003) Radiation hazard in space, Astrophysics and Space Science Library, vol 297. Springer, Dordrecht. https://doi.org/10.1007/978-94-017-0301-7

Mishra W, Srivastava N (2013) Estimating the arrival time of Earth-directed coronal mass ejections at in situ spacecraft using COR and HI observations from STEREO. Astrophys J 772(1):70. https://doi. org/10.1088/0004-637X/772/1/70. arXiv:1306.1397 [astro-ph.SR]

Miteva R, Klein KL, Kienreich I, Temmer M, Veronig A, Malandraki OE (2014) Solar energetic particles and associated EIT disturbances in solar cycle 23. Sol Phys 289(7):2601-2631. https://doi.org/10. 1007/s11207-014-0499-5. arXiv:1402.1676 [astro-ph.SR]

Miyoshi Y, Kataoka R, Kasahara Y, Kumamoto A, Nagai T, Thomsen MF (2013) High-speed solar wind with southward interplanetary magnetic field causes relativistic electron flux enhancement of the outer radiation belt via enhanced condition of whistler waves. Geophys Res Lett 40(17):4520-4525. https://doi.org/10.1002/grl.50916

Moore RL, Sterling AC, Hudson HS, Lemen JR (2001) Onset of the magnetic explosion in solar flares and coronal mass ejections. Astrophys J 552(2):833-848. https://doi.org/10.1086/320559

Moore RL, Sterling AC, Suess ST (2007) The width of a solar coronal mass ejection and the source of the driving magnetic explosion: a test of the standard scenario for CME production. Astrophys $\mathrm{J}$ 668(2):1221-1231. https://doi.org/10.1086/521215

Morosan DE, Palmerio E, Pomoell J, Vainio R, Palmroth M, Kilpua EKJ (2020) Three-dimensional reconstruction of multiple particle acceleration regions during a coronal mass ejection. Astron Astrophys 635:A62. https://doi.org/10.1051/0004-6361/201937133. arXiv:2001.08473 [astroph.SR]

Möstl C, Miklenic C, Farrugia CJ, Temmer M, Veronig A, Galvin AB, Vršnak B, Biernat HK (2008) Two-spacecraft reconstruction of a magnetic cloud and comparison to its solar source. Ann Geophys 26(10):3139-3152. https://doi.org/10.5194/angeo-26-3139-2008

Möstl C, Farrugia CJ, Temmer M, Miklenic C, Veronig AM, Galvin AB, Leitner M, Biernat HK (2009) Linking remote imagery of a coronal mass ejection to its in situ signatures at 1 AU. Astrophys J Lett 705(2):L180-L185. https://doi.org/10.1088/0004-637X/705/2/L180arXiv:0910.1188 [astro-ph.SR]

Möstl C, Temmer M, Rollett T, Farrugia CJ, Liu Y, Veronig AM, Leitner M, Galvin AB, Biernat HK (2010) STEREO and wind observations of a fast ICME flank triggering a prolonged geomagnetic storm on 5-7 April 2010. Geophys Res Lett 37:L24103. https://doi.org/10.1029/2010GL045175. arXiv: 1010.4150

Möstl C, Amla K, Hall JR, Liewer PC, De Jong EM, Colaninno RC, Veronig AM, Rollett T, Temmer M, Peinhart V, Davies JA, Lugaz N, Liu YD, Farrugia CJ, Luhmann JG, Vršnak B, Harrison RA, Galvin AB (2014) Connecting speeds, directions and arrival times of 22 coronal mass ejections from the Sun to 1 AU. Astrophys J 787(2):119. https://doi.org/10.1088/0004-637X/787/2/119. arXiv: 1404.3579 [astro-ph.SR]

Möstl C, Rollett T, Frahm RA, Liu YD, Long DM, Colaninno RC, Reiss MA, Temmer M, Farrugia CJ, Posner A, Dumbović M, Janvier M, Démoulin P, Boakes P, Devos A, Kraaikamp E, Mays ML, 
Vršnak B (2015) Strong coronal channelling and interplanetary evolution of a solar storm up to Earth and Mars. Nat Commun 6:7135. https://doi.org/10.1038/ncomms8135. arXiv:1506.02842 [astro-ph.SR]

Möstl C, Isavnin A, Boakes PD, Kilpua EKJ, Davies JA, Harrison RA, Barnes D, Krupar V, Eastwood JP, Good SW, Forsyth RJ, Bothmer V, Reiss MA, Amerstorfer T, Winslow RM, Anderson BJ, Philpott LC, Rodriguez L, Rouillard AP, Gallagher P, Nieves-Chinchilla T, Zhang TL (2017) Modeling observations of solar coronal mass ejections with heliospheric imagers verified with the heliophysics system observatory. Space Weather 15(7):955-970. https://doi.org/10.1002/2017SW001614. arXiv: 1703.00705 [astro-ph.SR]

Müller D, Zouganelis I, St Cyr OC, Gilbert HR, Nieves-Chinchilla T (2020) Europe's next mission to the Sun. Nat Astron 4:205. https://doi.org/10.1038/s41550-020-1015-5

Mulligan T, Russell CT, Luhmann JG (1998) Solar cycle evolution of the structure of magnetic clouds in the inner heliosphere. Geophys Res Lett 25(15):2959-2962. https://doi.org/10.1029/98GL01302

Murray SA, Guerra JA, Zucca P, Park SH, Carley EP, Gallagher PT, Vilmer N, Bothmer V (2018) Connecting coronal mass ejections to their solar active region sources: combining results from the HELCATS and FLARECAST projects. Sol Phys 293(4):60. https://doi.org/10.1007/s11207-0181287-4. arXiv:1803.06529 [astro-ph.SR]

Neupert WM (1968) Comparison of solar X-ray line emission with microwave emission during flares. Astrophys J Lett 153:L59. https://doi.org/10.1086/180220

Ngwira CM, Pulkkinen A, Kuznetsova MM, Glocer A (2014) Modeling extreme "Carrington-type", space weather events using three-dimensional global MHD simulations. J Geophys Res 119(6):4456-4474. https://doi.org/10.1002/2013JA019661

Nieves-Chinchilla T, Colaninno R, Vourlidas A, Szabo A, Lepping RP, Boardsen SA, Anderson BJ, Korth H (2012) Remote and in situ observations of an unusual Earth-directed coronal mass ejection from multiple viewpoints. J Geophys Res 117(A6):A06106. https://doi.org/10.1029/2011JA017243

Nitta NV, Mulligan T (2017) Earth-affecting coronal mass ejections without obvious low coronal signatures. Sol Phys 292(9):125. https://doi.org/10.1007/s11207-017-1147-7

Odstrčil D, Pizzo VJ (1999) Three-dimensional propagation of CMEs in a structured solar wind flow: 1. CME launched within the streamer belt. J Geophys Res 104(A1):483-492. https://doi.org/10.1029/ 1998JA900019

Ogilvie KW, Chornay DJ, Fritzenreiter RJ, Hunsaker F, Keller J, Lobell J, Miller G, Scudder JD, Sittler JEC, Torbert RB, Bodet D, Needell G, Lazarus AJ, Steinberg JT, Tappan JH, Mavretic A, Gergin E (1995) SWE, a comprehensive plasma instrument for the Wind spacecraft. Space Sci Rev 71(1-4):55-77. https://doi.org/10.1007/BF00751326

Olmedo O, Zhang J (2010) Partial torus instability. Astrophys J 718(1):433-440. https://doi.org/10.1088/ 0004-637X/718/1/433

Omodei N, Pesce-Rollins M, Longo F, Allafort A, Krucker S (2018) Fermi-LAT observations of the 2017 September 10 solar flare. Astrophys J Lett 865(1):L7. https://doi.org/10.3847/2041-8213/aae077. arXiv:1803.07654 [astro-ph.HE]

Ontiveros V, Vourlidas A (2009) Quantitative measurements of coronal mass ejection-driven shocks from LASCO observations. Astrophys J 693(1):267-275. https://doi.org/10.1088/0004-637X/693/1/267. arXiv:0811.3743 [astro-ph]

Opitz A, Fedorov A, Wurz P, Szego K, Sauvaud JA, Karrer R, Galvin AB, Barabash S, Ipavich F (2010) Solar-wind bulk velocity throughout the inner heliosphere from multi-spacecraft measurements. Sol Phys 264(2):377-382. https://doi.org/10.1007/s11207-010-9583-7

Owens MJ, Forsyth RJ (2013) The heliospheric magnetic field. Living Rev Sol Phys 10:5. https://doi.org/ 10.12942/lrsp-2013-5

Owens MJ, Challen R, Methven J, Henley E, Jackson DR (2013) A 27 day persistence model of nearEarth solar wind conditions: a long lead-time forecast and a benchmark for dynamical models. Space Weather 11(5):225-236. https://doi.org/10.1002/swe.20040

Owens MJ, Riley P, Lang M, Lockwood M (2019) Near-Earth solar wind forecasting using corotation from L5: the error introduced by heliographic latitude offset. Space Weather 17(7):1105-1113. https://doi.org/10.1029/2019SW002204

Paassilta M, Papaioannou A, Dresing N, Vainio R, Valtonen E, Heber B (2018) Catalogue of > $55 \mathrm{MeV}$ wide-longitude solar proton events observed by SOHO, ACE, and the STEREOs at $\approx 1$ AU during 2009-2016. Sol Phys 293(4):70. https://doi.org/10.1007/s11207-018-1284-7. arXiv:1803.05370 [physics.space-ph] 
Palmerio E, Kilpua EKJ, James AW, Green LM, Pomoell J, Isavnin A, Valori G (2017) Determining the intrinsic CME flux rope type using remote-sensing solar disk observations. Sol Phys 292(2):39. https://doi.org/10.1007/s11207-017-1063-X. arXiv:1701.08595 [astro-ph.SR]

Palmerio E, Kilpua EKJ, Möstl C, Bothmer V, James AW, Green LM, Isavnin A, Davies JA, Harrison RA (2018) Coronal magnetic structure of earthbound CMEs and in situ comparison. Space Weather 16(5):442-460. https://doi.org/10.1002/2017SW001767. arXiv:1803.04769 [astro-ph.SR]

Panasenco O, Martin SF, Velli M, Vourlidas A (2013) Origins of rolling, twisting, and non-radial propagation of eruptive solar events. Sol Phys 287(1-2):391-413. https://doi.org/10.1007/s11207012-0194-3. arXiv:1211.1376 [astro-ph.SR]

Papaioannou A, Sandberg I, Anastasiadis A, Kouloumvakos A, Georgoulis MK, Tziotziou K, Tsiropoula G, Jiggens P, Hilgers A (2016) Solar flares, coronal mass ejections and solar energetic particle event characteristics. J Space Weather Space Clim 6:A42. https://doi.org/10.1051/swsc/2016035

Parenti S (2014) Solar prominences: observations. Living Rev Sol Phys 11:1. https://doi.org/10.12942/ lrsp-2014-1

Park J, Innes DE, Bucik R, Moon YJ (2013) The source regions of solar energetic particles detected by widely separated spacecraft. Astrophys J 779(2):184. https://doi.org/10.1088/0004-637X/779/2/184

Patsourakos S, Vourlidas A (2009) "Extreme ultraviolet waves" are waves: first quadrature observations of an extreme ultraviolet wave from STEREO. Astrophys J Lett 700(2):L182-L186. https://doi.org/ 10.1088/0004-637X/700/2/L182. arXiv:0905.2164 [astro-ph.SR]

Patsourakos S, Vourlidas A, Kliem B (2010) Toward understanding the early stages of an impulsively accelerated coronal mass ejection. SECCHI observations. Astron Astrophys 522:A100. https://doi. org/10.1051/0004-6361/200913599arXiv:1008.1171 [astro-ph.SR]

Patsourakos S, Georgoulis MK, Vourlidas A, Nindos A, Sarris T, Anagnostopoulos G, Anastasiadis A, Chintzoglou G, Daglis IA, Gontikakis C, Hatzigeorgiu N, Iliopoulos AC, Katsavrias C, Kouloumvakos A, Moraitis K, Nieves-Chinchilla T, Pavlos G, Sarafopoulos D, Syntelis P, Tsironis C, Tziotziou K, Vogiatzis II, Balasis G, Georgiou M, Karakatsanis LP, Malandraki OE, Papadimitriou C, Odstrčil D, Pavlos EG, Podlachikova O, Sand berg I, Turner DL, Xenakis MN, Sarris E, Tsinganos K, Vlahos L (2016) The major geoeffective solar eruptions of 2012 March 7: comprehensive Sun-to-Earth analysis. Astrophys J 817(1):14. https://doi.org/10.3847/0004-637X/ $817 / 1 / 14$

Pesnell WD, Thompson BJ, Chamberlin PC (2012) The Solar Dynamics Observatory (SDO). Sol Phys 275:3-15. https://doi.org/10.1007/s11207-011-9841-3

Petrosian V, Liu S (2004) Stochastic acceleration of electrons and protons. I. Acceleration by parallelpropagating waves. Astrophys J 610(1):550-571. https://doi.org/10.1086/421486. arXiv:astro-ph/ 0401585 [astro-ph]

Pirjola R, Viljanen A, Pulkkinen A, Kilpua S, Amm O (2005) Ground effects of space weather. In: Daglis IA (ed) Effects of space weather on technology infrastructure. Springer, Dordrecht, pp 235-256

Pizzo V (1978) A three-deminsional model of corotating streams in the solar wind 1. Theoretical foundations. J Geophys Res 83(A12):5563-5572. https://doi.org/10.1029/JA083iA12p05563

Plotnikov I, Rouillard AP, Share GH (2017) The magnetic connectivity of coronal shocks from behindthe-limb flares to the visible solar surface during $\gamma$-ray events. Astron Astrophys 608:A43. https:// doi.org/10.1051/0004-6361/201730804

Pomoell J, Poedts S (2018) EUHFORIA: European heliospheric forecasting information asset. J Space Weather Space Clim 8:A35. https://doi.org/10.1051/swsc/2018020

Pomoell J, Vainio R, Kissmann R (2008) MHD modeling of coronal large-amplitude waves related to CME lift-off. Sol Phys 253(1-2):249-261. https://doi.org/10.1007/s11207-008-9186-8

Pulkkinen T (2007) Space weather: terrestrial perspective. Living Rev Sol Phys 4:1. https://doi.org/10. 12942/lrsp-2007-1

Reames DV (1999) Particle acceleration at the Sun and in the heliosphere. Space Sci Rev 90:413-491. https://doi.org/10.1023/A:1005105831781

Reames DV (2009) Solar release times of energetic particles in ground-level events. Astrophys J 693(1):812-821. https://doi.org/10.1088/0004-637X/693/1/812

Reames DV (2017) Solar energetic particles: a modern primer on understanding sources, acceleration and propagation, Lecture Notes in Physics, vol 932. Springer. https://doi.org/10.1007/978-3-319-508719

Reames DV, Barbier LM, Ng CK (1996) The spatial distribution of particles accelerated by coronal mass ejection-driven shocks. Astrophys J 466:473. https://doi.org/10.1086/177525 
Reeves GD, McAdams KL, Friedel RHW, O'Brien TP (2003) Acceleration and loss of relativistic electrons during geomagnetic storms. Geophys Res Lett 30(10):1529. https://doi.org/10.1029/ 2002GL016513

Reeves KK, Linker JA, Mikić Z, Forbes TG (2010) Current sheet energetics, flare emissions, and energy partition in a simulated solar eruption. Astrophys J 721(2):1547-1558. https://doi.org/10.1088/0004$637 \mathrm{X} / 721 / 2 / 1547$

Reiner MJ, Goetz K, Fainberg J, Kaiser ML, Maksimovic M, Cecconi B, Hoang S, Bale SD, Bougeret JL (2009) Multipoint observations of solar type III radio bursts from STEREO and wind. Sol Phys 259(1-2):255-276. https://doi.org/10.1007/s11207-009-9404-z

Reiss MA, Temmer M, Veronig AM, Nikolic L, Vennerstrom S, Schöngassner F, Hofmeister SJ (2016) Verification of high-speed solar wind stream forecasts using operational solar wind models. Space Weather. https://doi.org/10.1002/2016SW001390

Richardson IG (2018) Solar wind stream interaction regions throughout the heliosphere. Living Rev Sol Phys 15:1. https://doi.org/10.1007/s41116-017-0011-z

Richardson IG, Cane HV (1995) Regions of abnormally low proton temperature in the solar wind (1965-1991) and their association with ejecta. J Geophys Res 100(A12):23397-23412. https://doi. org/10.1029/95JA02684

Richardson IG, Cane HV (2010) Near-Earth interplanetary coronal mass ejections during solar cycle 23 (1996-2009): Catalog and Summary of Properties. Sol Phys 264(1):189-237. https://doi.org/10. $1007 / \mathrm{s} 11207-010-9568-6$

Richardson IG, Cane HV (2012) Near-Earth solar wind flows and related geomagnetic activity during more than four solar cycles (1963-2011). J Space Weather Space Clim 2:A02. https://doi.org/10. $1051 /$ swsc/2012003

Richardson IG, Cliver EW, Cane HV (2001) Sources of geomagnetic storms for solar minimum and maximum conditions during 1972-2000. Geophys Res Lett 28(13):2569-2572. https://doi.org/10. 1029/2001GL013052

Richardson IG, von Rosenvinge TT, Cane HV (2015) The properties of solar energetic particle eventassociated coronal mass ejections reported in different CME catalogs. Sol Phys 290(6):1741-1759. https://doi.org/10.1007/s11207-015-0701-4. arXiv:1505.03071 [astro-ph.SR]

Riley P, Crooker NU (2004) Kinematic treatment of coronal mass ejection evolution in the solar wind. Astrophys J 600(2):1035-1042. https://doi.org/10.1086/379974

Riley P, Lionello R, Linker JA, Mikic Z, Luhmann J, Wijaya J (2011) Global MHD modeling of the solar corona and inner heliosphere for the whole heliosphere interval. Sol Phys 274(1-2):361-377. https:// doi.org/10.1007/s11207-010-9698-x

Riley P, Linker JA, Lionello R, Mikić Z (2012) Corotating interaction regions during the recent solar minimum: the power and limitations of global MHD modeling. J Atmos Sol-Terr Phys 83:1-10. https://doi.org/10.1016/j.jastp.2011.12.013

Riley P, Mays ML, Andries J, Amerstorfer T, Biesecker D, Delouille V, Dumbović M, Feng X, Henley E, Linker JA, Möstl C, Nuñez M, Pizzo V, Temmer M, Tobiska WK, Verbeke C, West MJ, Zhao X (2018) Forecasting the arrival time of coronal mass ejections: analysis of the CCMC CME scoreboard. Space Weather 16(9):1245-1260. https://doi.org/10.1029/2018SW001962 . arXiv:1810. 07289 [physics.space-ph]

Robbrecht E, Patsourakos S, Vourlidas A (2009) No trace left behind: STEREO observation of a coronal mass ejection without low coronal signatures. Astrophys J 701(1):283-291. https://doi.org/10.1088/ 0004-637X/701/1/283. arXiv:0905.2583 [astro-ph.SR]

Rochus P, Auchere F, Berghmans D et al (2020) The solar orbiter EUI instrument: the extreme ultraviolet imager. Astron Astrophys. https://doi.org/10.1051/0004-6361/201936663

Rollett T, Möstl C, Temmer M, Veronig AM, Farrugia CJ, Biernat HK (2012) Constraining the kinematics of coronal mass ejections in the inner heliosphere with in-situ signatures. Sol Phys 276(1-2):293-314. https://doi.org/10.1007/s11207-011-9897-0. arXiv:1110.0300 [astro-ph.SR]

Rollett T, Möstl C, Isavnin A, Davies JA, Kubicka M, Amerstorfer UV, Harrison RA (2016) ElEvoHI: a novel CME prediction tool for heliospheric imaging combining an elliptical front with drag-based model fitting. Astrophys J 824(2):131. https://doi.org/10.3847/0004-637X/824/2/131. arXiv:1605. 00510 [astro-ph.SR]

Rosenbauer H, Schwenn R, Marsch E, Meyer B, Miggenrieder H, Montgomery MD, Muehlhaeuser KH, Pilipp W, Voges W, Zink SM (1977) A survey on initial results of the Helios plasma experiment. J Geophys/Z Geophys 42(6):561-580 
Rotter T, Veronig AM, Temmer M, Vršnak B (2012) Relation between coronal hole areas on the sun and the solar wind parameters at 1 AU. Sol Phys 281(2):793-813. https://doi.org/10.1007/s11207-012$0101-y$

Rouillard AP (2011) Relating white light and in situ observations of coronal mass ejections: a review. J Atmos Sol-Terr Phys 73(10):1201-1213. https://doi.org/10.1016/j.jastp.2010.08.015

Rouillard AP, Davies JA, Forsyth RJ, Rees A, Davis CJ, Harrison RA, Lockwood M, Bewsher D, Crothers SR, Eyles CJ, Hapgood M, Perry CH (2008) First imaging of corotating interaction regions using the STEREO spacecraft. Geophys Res Lett 35(10):L10110. https://doi.org/10.1029/ 2008GL033767

Rouillard AP, Lavraud B, Sheeley NR, Davies JA, Burlaga LF, Savani NP, Jacquey C, Forsyth RJ (2010) White light and in situ comparison of a forming merged interaction region. Astrophys $\mathrm{J}$ 719(2):1385-1392. https://doi.org/10.1088/0004-637X/719/2/1385

Rouillard AP, Sheeley NR, Tylka A, Vourlidas A, Ng CK, Rakowski C, Cohen CMS, Mewaldt RA, Mason GM, Reames D, Savani NP, StCyr OC, Szabo A (2012) The longitudinal properties of a solar energetic particle event investigated using modern solar imaging. Astrophys J 752(1):44. https://doi. org/10.1088/0004-637X/752/1/44

Rouillard AP, Plotnikov I, Pinto RF, Tirole M, Lavarra M, Zucca P, Vainio R, Tylka AJ, Vourlidas A, De Rosa ML, Linker J, Warmuth A, Mann G, Cohen CMS, Mewaldt RA (2016) Deriving the properties of coronal pressure fronts in 3D: application to the 2012 May 17 Ground Level Enhancement. Astrophys J 833(1):45. https://doi.org/10.3847/1538-4357/833/1/45. arXiv:1605.05208 [astroph.SR]

Ruffenach A, Lavraud B, Farrugia CJ, Démoulin P, Dasso S, Owens MJ, Sauvaud JA, Rouillard AP, Lynnyk A, Foullon C, Savani NP, Luhmann JG, Galvin AB (2015) Statistical study of magnetic cloud erosion by magnetic reconnection. J Geophys Res 120(1):43-60. https://doi.org/10.1002/ 2014JA020628

Sachdeva N, Subramanian P, Colaninno R, Vourlidas A (2015) CME propagation: where does aerodynamic drag "take over"? Astrophys J 809(2):158. https://doi.org/10.1088/0004-637X/809/2/ 158. arXiv:1507.05199 [astro-ph.SR]

Salman TM, Winslow RM, Lugaz N (2020) Radial evolution of coronal mass ejections between MESSENGER, venus express, STEREO, and L1: catalog and analysis. J Geophys Res 125(1):e27084. https://doi.org/10.1029/2019JA027084. arXiv:1912.11731 [physics.space-ph]

Sammis I, Tang F, Zirin H (2000) The dependence of large flare occurrence on the magnetic structure of sunspots. Astrophys J 540(1):583-587. https://doi.org/10.1086/309303

Santandrea S, Gantois K, Strauch K, Teston F, Tilmans E, Baijot C, Gerrits D, De Groof A, Schwehm G, Zender J (2013) PROBA2: mission and spacecraft overview. Sol Phys 286(1):5-19. https://doi.org/ 10.1007/s11207-013-0289-5

Savani NP, Vourlidas A, Szabo A, Mays ML, Richardson IG, Thompson BJ, Pulkkinen A, Evans R, Nieves-Chinchilla T (2015) Predicting the magnetic vectors within coronal mass ejections arriving at Earth: 1. Initial architecture. Space Weather 13(6):374-385. https://doi.org/10.1002/ 2015SW001171. arXiv:1502.02067 [astro-ph.EP]

Schmieder B, Aulanier G, Vršnak B (2015) Flare-CME models: an observational perspective (invited review). Sol Phys 290(12):3457-3486. https://doi.org/10.1007/s11207-015-0712-1

Schrijver CJ (2007) A characteristic magnetic field pattern associated with all major solar flares and its use in flare forecasting. Astrophys J Lett 655(2):L117-L120. https://doi.org/10.1086/511857

Schrijver CJ, De Rosa ML (2003) Photospheric and heliospheric magnetic fields. Sol Phys 212(1):165-200. https://doi.org/10.1023/A:1022908504100

Schwenn R (1983) Direct correlations between coronal transients and interplanetary disturbances. Space Sci Rev 34(1):85-99. https://doi.org/10.1007/BF00221199

Schwenn R (2006) Space weather: the solar perspective. Living Rev Sol Phys 3:2. https://doi.org/10. 12942/lrsp-2006-2

Schwenn R, Rosenbauer H, Miggenrieder H (1975) Das Plasmaexperiment auf Helios (E1). Raumfahrtforschung 19:226-232

Schwenn R, dal Lago A, Huttunen E, Gonzalez WD (2005) The association of coronal mass ejections with their effects near the Earth. Ann Geophys 23(3):1033-1059. https://doi.org/10.5194/angeo-231033-2005

Scolini C, Rodriguez L, Mierla M, Pomoell J, Poedts S (2019) Observation-based modelling of magnetised coronal mass ejections with EUHFORIA. Astron Astrophys 626:A122. https://doi.org/ 10.1051/0004-6361/201935053. arXiv:1904.07059 [astro-ph.SR] 
Scolini C, Chané E, Temmer M, Kilpua EKJ, Dissauer K, Veronig AM, Palmerio E, Pomoell J, Dumbović M, Guo J, Rodriguez L, Poedts S (2020) CME-CME interactions as sources of CME geoeffectiveness: the formation of the complex ejecta and intense geomagnetic storm in 2017 early September. Astrophys J Suppl Ser 247(1):21. https://doi.org/10.3847/1538-4365/ab6216. arXiv: 1911.10817 [astro-ph.SR]

Seaton DB, Darnel JM (2018) Observations of an eruptive solar flare in the extended EUV solar corona. Astrophys J Lett 852(1):L9. https://doi.org/10.3847/2041-8213/aaa28e. arXiv:1712.06003 [astroph.SR]

Shanmugaraju A, Vršnak B (2014) Transit time of coronal mass ejections under different ambient solar wind conditions. Sol Phys 289(1):339-349. https://doi.org/10.1007/s11207-013-0322-8

Sheeley NR, Wang YM, Hawley SH, Brueckner GE, Dere KP, Howard RA, Koomen MJ, Korendyke CM, Michels DJ, Paswaters SE, Socker DG, St Cyr OC, Wang D, Lamy PL, Llebaria A, Schwenn R, Simnett GM, Plunkett S, Biesecker DA (1997) Measurements of flow speeds in the corona between 2 and $30 R_{\odot}$. Astrophys J 484(1):472-478. https://doi.org/10.1086/304338

Shen C, Wang Y, Ye P, Wang S (2006) Is there any evident effect of coronal holes on gradual solar energetic particle events? Astrophys J 639(1):510-515. https://doi.org/10.1086/499199

Shen C, Wang Y, Gui B, Ye P, Wang S (2011) Kinematic evolution of a slow CME in corona viewed by STEREO-B on 8 October 2007. Sol Phys 269(2):389-400. https://doi.org/10.1007/s11207-0119715-8. arXiv:1101.3205 [astro-ph.SR]

Shen C, Wang Y, Pan Z, Zhang M, Ye P, Wang S (2013) Full halo coronal mass ejections: do we need to correct the projection effect in terms of velocity? J Geophys Res 118(11):6858-6865. https://doi.org/ 10.1002/2013JA018872. arXiv:1303.4059 [astro-ph.SR]

Shen C, Xu M, Wang Y, Chi Y, Luo B (2018) Why the shock-ICME complex structure is important: learning from the early 2017 September CMEs. Astrophys J 861(1):28. https://doi.org/10.3847/ 1538-4357/aac204. arXiv:1805.05763 [physics.space-ph]

Shi T, Wang Y, Wan L, Cheng X, Ding M, Zhang J (2015) Predicting the arrival time of coronal mass ejections with the graduated cylindrical shell and drag force model. Astrophys J 806(2):271. https:// doi.org/10.1088/0004-637X/806/2/271. arXiv:1505.00884 [astro-ph.SR]

Shibata K, Magara T (2011) Solar flares: magnetohydrodynamic processes. Living Rev Sol Phys 8:6. https://doi.org/10.12942/lrsp-2011-6

Shiota D, Kataoka R (2016) Magnetohydrodynamic simulation of interplanetary propagation of multiple coronal mass ejections with internal magnetic flux rope (SUSANOO-CME). Space Weather 14(2):56-75. https://doi.org/10.1002/2015SW001308

Singh T, Yalim MS, Pogorelov NV, Gopalswamy N (2019) Simulating solar coronal mass ejections constrained by observations of their speed and poloidal flux. Astrophys J Lett 875(2):L17. https:// doi.org/10.3847/2041-8213/ab14e9arXiv:1904.00140 [astro-ph.SR]

St Cyr OC, Plunkett SP, Michels DJ, Paswaters SE, Koomen MJ, Simnett GM, Thompson BJ, Gurman JB, Schwenn R, Webb DF, Hildner E, Lamy PL (2000) Properties of coronal mass ejections: SOHO LASCO observations from January 1996 to June 1998. J Geophys Res 105(A8):18169-18186. https://doi.org/10.1029/1999JA000381

St Cyr OC, Posner A, Burkepile JT (2017) Solar energetic particle warnings from a coronagraph. Space Weather 15(1):240-257. https://doi.org/10.1002/2016SW001545

Stone EC, Frandsen AM, Mewaldt RA, Christian ER, Margolies D, Ormes JF, Snow F (1998) The advanced composition explorer. Space Sci Rev 86:1-22. https://doi.org/10.1023/A:1005082526237

Sturrock PA (1966) Model of the high-energy phase of solar flares. Nature 211(5050):695-697. https:// doi.org/10.1038/211695a0

Sun X, Bobra MG, Hoeksema JT, Liu Y, Li Y, Shen C, Couvidat S, Norton AA, Fisher GH (2015) Why is the great solar active region 12192 flare-rich but CME-poor? Astrophys J Lett 804(2):L28. https:// doi.org/10.1088/2041-8205/804/2/L28. arXiv:1502.06950 [astro-ph.SR]

Tandberg-Hanssen E (1995) The nature of solar prominences. Astrophysics and Space Science Library, vol 199. Springer, Dordrecht. https://doi.org/10.1007/978-94-017-3396-0

Temmer M (2016) Kinematical properties of coronal mass ejections. Astron Nachr 337(10):1010. https:// doi.org/10.1002/asna.201612425. arXiv:1603.01398 [astro-ph.SR]

Temmer M, Nitta NV (2015) Interplanetary Propagation Behavior of the Fast Coronal Mass Ejection on 23 July 2012. Sol Phys 290(3):919-932. https://doi.org/10.1007/s11207-014-0642-3. arXiv:1411. 6559 [astro-ph.SR] 
Temmer M, Veronig AM, Vršnak B, Rybák J, Gömöry P, Stoiser S, Maričić D (2008) Acceleration in fast halo CMEs and synchronized flare HXR bursts. Astrophys J Lett 673(1):L95. https://doi.org/10. $1086 / 527414$

Temmer M, Preiss S, Veronig AM (2009) CME projection effects studied with STEREO/COR and SOHO/LASCO. Sol Phys 256(1-2):183-199. https://doi.org/10.1007/s11207-009-9336-7

Temmer M, Veronig AM, Kontar EP, Krucker S, Vršnak B (2010) Combined STEREO/RHESSI study of coronal mass ejection acceleration and particle acceleration in solar flares. Astrophys $\mathrm{J}$ 712(2):1410-1420. https://doi.org/10.1088/0004-637X/712/2/1410. arXiv:1002.3080 [astro-ph.SR]

Temmer M, Reiss MA, Nikolic L, Hofmeister SJ, Veronig AM (2017a) Preconditioning of interplanetary space due to transient CME disturbances. Astrophys J 835(2):141. https://doi.org/10.3847/15384357/835/2/141. arXiv:1612.06080 [astro-ph.SR]

Temmer M, Thalmann JK, Dissauer K, Veronig AM, Tschernitz J, Hinterreiter J, Rodriguez L (2017b) On flare-CME characteristics from Sun to Earth combining remote-sensing image data with in situ measurements supported by modeling. Sol Phys 292(7):93. https://doi.org/10.1007/s11207-0171112-5. arXiv:1703.00694 [astro-ph.SR]

Temmer M, Hinterreiter J, Reiss MA (2018) Coronal hole evolution from multi-viewpoint data as input for a STEREO solar wind speed persistence model. J Space Weather Space Clim 8:A18. https://doi. org/10.1051/swsc/2018007. arXiv:1801.10213 [astro-ph.SR]

Temmer M, Holzknecht L, Dumbović M, Vršnak B, Sachdeva N, Heinemann SG, Dissauer K, Scolini C, Asvestari E, Veronig AM, Hofmeister SJ (2021) Deriving CME density from remote sensing data and comparison to in-situ measurements. J Geophys Res. https://doi.org/10.1029/2020ja028380

Thalmann JK, Su Y, Temmer M, Veronig AM (2015) The confined X-class flares of solar active region 2192. Astrophys J Lett 801(2):L23. https://doi.org/10.1088/2041-8205/801/2/L23. arXiv:1502. 05157 [astro-ph.SR]

Thernisien A, Vourlidas A, Howard RA (2009) Forward modeling of coronal mass ejections using STEREO/SECCHI data. Sol Phys 256(1-2):111-130. https://doi.org/10.1007/s11207-009-9346-5

Thernisien AFR, Howard RA, Vourlidas A (2006) Modeling of flux rope coronal mass ejections. Astrophys J 652(1):763-773. https://doi.org/10.1086/508254

Thompson BJ, Plunkett SP, Gurman JB, Newmark JS, St Cyr OC, Michels DJ (1998) SOHO/EIT observations of an Earth-directed coronal mass ejection on May 12, 1997. Geophys Res Lett 25(14):2465-2468. https://doi.org/10.1029/98GL50429

Toriumi S, Wang H (2019) Flare-productive active regions. Living Rev Sol Phys 16:3. https://doi.org/10. 1007/s41116-019-0019-7. arXiv:1904.12027 [astro-ph.SR]

Toriumi S, Schrijver CJ, Harra LK, Hudson H, Nagashima K (2017) Magnetic properties of solar active regions that govern large solar flares and eruptions. Astrophys J 834(1):56. https://doi.org/10.3847/ 1538-4357/834/1/56. arXiv:1611.05047 [astro-ph.SR]

Török T, Kliem B (2005) Confined and ejective eruptions of kink-unstable flux ropes. Astrophys J Lett 630(1):L97-L100. https://doi.org/10.1086/462412. arXiv:astro-ph/0507662 [astro-ph]

Török T, Temmer M, Valori G, Veronig AM, van Driel-Gesztelyi L, Vršnak B (2013) Initiation of coronal mass ejections by sunspot rotation. Sol Phys 286(2):453-477. https://doi.org/10.1007/ s11207-013-0269-9. arXiv:1401.2922 [astro-ph.SR]

Tousey R (1971) Chromosphere and corona: observations of the extreme ultraviolet solar spectrum. Philos Trans R Soc Lond Ser A 270(1202):59-70. https://doi.org/10.1098/rsta.1971.0060

Tripathi D, Bothmer V, Cremades H (2004) The basic characteristics of EUV post-eruptive arcades and their role as tracers of coronal mass ejection source regions. Astron Astrophys 422:337-349. https:// doi.org/10.1051/0004-6361:20035815

Trottet G, Samwel S, Klein KL, Dudok de Wit T, Miteva R (2015) Statistical evidence for contributions of flares and coronal mass ejections to major solar energetic particle events. Sol Phys 290(3):819-839. https://doi.org/10.1007/s11207-014-0628-1. arXiv:1411.4133 [astro-ph.SR]

Tschernitz J, Veronig AM, Thalmann JK, Hinterreiter J, Pötzi W (2018) Reconnection fluxes in eruptive and confined flares and implications for superflares on the Sun. Astrophys J 853(1):41. https://doi. org/10.3847/1538-4357/aaa199. arXiv:1712.04701 [astro-ph.SR]

Tsurutani BT, Gonzalez WD, Gonzalez ALC, Guarnieri FL, Gopalswamy N, Grande M, Kamide Y, Kasahara Y, Lu G, Mann I, McPherron R, Soraas F, Vasyliunas V (2006) Corotating solar wind streams and recurrent geomagnetic activity: a review. J Geophys Res 111(A7):A07S01. https://doi. org/10.1029/2005JA011273

van Driel-Gesztelyi L, Green LM (2015) Evolution of active regions. Living Rev Sol Phys 12:1. https:// doi.org/10.1007//rsp-2015-1 
van Haarlem MP, Wise MW, Gunst AW, Heald G, McKean JP, Hessels JWT, de Bruyn AG, Nijboer R et al (2013) LOFAR: the low-frequency array. Astron Astrophys 556:A2. https://doi.org/10.1051/ 0004-6361/201220873. arXiv:1305.3550 [astro-ph.IM]

Vennerstrom S, Lefevre L, Dumbović M, Crosby N, Malandraki O, Patsou I, Clette F, Veronig A, Vršnak B, Leer K, Moretto T (2016) Extreme geomagnetic storms-1868-2010. Sol Phys 291(5):1447-1481. https://doi.org/10.1007/s11207-016-0897-y

Venzmer MS, Bothmer V (2018) Solar-wind predictions for the Parker Solar Probe orbit. Near-Sun extrapolations derived from an empirical solar-wind model based on helios and OMNI observations. Astron Astrophys 611:A36. https://doi.org/10.1051/0004-6361/201731831. arXiv:1711.07534 [astro-ph.SR]

Verbanac G, Živković S, Vršnak B, Bandić M, Hojsak T (2013) Comparison of geoeffectiveness of coronal mass ejections and corotating interaction regions. Astron Astrophys 558:A85. https://doi. org/10.1051/0004-6361/201220417

Verbeke C, Mays ML, Temmer M, Bingham S, Steenburgh R, Dumbović M, Núñez M, Jian LK, Hess P, Wiegand C, Taktakishvili A, Andries J (2019) Benchmarking CME arrival time and impact: progress on metadata, metrics, and events. Space Weather 17(1):6-26. https://doi.org/10.1029/ 2018SW002046. arXiv:1811.10695 [astro-ph.SR]

Veronig AM, Muhr N, Kienreich IW, Temmer M, Vrnak B (2010) First observations of a dome-shaped large-scale coronal extreme-ultraviolet wave. Astrophys J Lett. https://doi.org/10.1088/2041-8205/ 716/1/L57

Veronig AM, Podladchikova T, Dissauer K, Temmer M, Seaton DB, Long D, Guo J, Vršnak B, Harra L, Kliem B (2018) Genesis and impulsive evolution of the 2017 September 10 coronal mass ejection. Astrophys J 868(2):107. https://doi.org/10.3847/1538-4357/aaeac5. arXiv:1810.09320 [astro-ph.SR]

Verscharen D, Klein KG, Maruca BA (2019) The multi-scale nature of the solar wind. Living Rev Sol Phys 16:5. https://doi.org/10.1007/s41116-019-0021-0. arXiv:1902.03448 [physics.space-ph]

Vial JC, Engvold O (2015) Solar prominences, Astrophysics and Space Science Library, vol 415. Springer, Cham. https://doi.org/10.1007/978-3-319-10416-4

Vlahos L, Anastasiadis A, Papaioannou A, Kouloumvakos A, Isliker H (2019) Sources of solar energetic particles. Philos Trans R Soc Lond Ser A 377(2148):20180095. https://doi.org/10.1098/rsta.2018. 0095. arXiv:1903.08200 [astro-ph.HE]

Vourlidas A, Howard RA (2006) The proper treatment of coronal mass ejection brightness: a new methodology and implications for observations. Astrophys J 642(2):1216-1221. https://doi.org/10. $1086 / 501122$

Vourlidas A, Webb DF (2018) Streamer-blowout coronal mass ejections: their properties and relation to the coronal magnetic field structure. Astrophys J 861(2):103. https://doi.org/10.3847/1538-4357/ aaca3e. arXiv:1806.00644 [astro-ph.SR]

Vourlidas A, Howard RA, Esfandiari E, Patsourakos S, Yashiro S, Michalek G (2010) Comprehensive analysis of coronal mass ejection mass and energy properties over a full solar cycle. Astrophys $\mathbf{J}$ 722(2):1522-1538. https://doi.org/10.1088/0004-637X/722/2/1522. arXiv:1008.3737 [astro-ph.SR]

Vourlidas A, Colaninno R, Nieves-Chinchilla T, Stenborg G (2011) The first observation of a rapidly rotating coronal mass ejection in the middle corona. Astrophys J Lett 733(2):L23. https://doi.org/10. 1088/2041-8205/733/2/L23

Vourlidas A, Lynch BJ, Howard RA, Li Y (2013) How many CMEs have flux ropes? Deciphering the signatures of shocks, flux ropes, and prominences in coronagraph observations of CMEs. Sol Phys 284(1):179-201. https://doi.org/10.1007/s11207-012-0084-8. arXiv:1207.1599 [astro-ph.SR]

Vourlidas A, Balmaceda LA, Stenborg G, Dal Lago A (2017) Multi-viewpoint coronal mass ejection catalog based on STEREO COR2 observations. Astrophys J 838(2):141. https://doi.org/10.3847/ 1538-4357/aa67f0

Vourlidas A, Patsourakos S, Savani NP (2019) Predicting the geoeffective properties of coronal mass ejections: current status, open issues and path forward. Philos Trans R Soc Lond Ser A 377(2148):20180096. https://doi.org/10.1098/rsta.2018.0096

Vršnak B, Maričić D, Stanger AL, Veronig AM, Temmer M, Roša D (2007) Acceleration phase of coronal mass ejections: I. Temporal and spatial scales. Sol Phys 241(1):12. https://doi.org/10.1007/ s11207-006-0290-3

Vršnak B (2001) Deceleration of coronal mass ejections. Sol Phys 202(1):173-189. https://doi.org/10. 1023/A:1011833114104

Vršnak B (2006) Forces governing coronal mass ejections. Adv Space Res 38(3):431-440. https://doi.org/ 10.1016/j.asr.2005.03.090 
Vršnak B (2008) Processes and mechanisms governing the initiation and propagation of CMEs. Ann Geophys 26(10):3089-3101. https://doi.org/10.5194/angeo-26-3089-2008

Vršnak B, Cliver EW (2008) Origin of coronal shock waves. Invited review. Sol Phys 253(1-2):215-235. https://doi.org/10.1007/s11207-008-9241-5

Vršnak B, Magdalenić J, Aurass H, Mann G (2002) Band-splitting of coronal and interplanetary type II bursts. II. Coronal magnetic field and Alfvén velocity. Astron Astrophys 396:673-682. https://doi. org/10.1051/0004-6361:20021413

Vršnak B, Temmer M, Veronig AM (2007) Coronal holes and solar wind high-speed streams: I. Forecasting the solar wind parameters. Sol Phys 240(2):315-330. https://doi.org/10.1007/s11207007-0285-8

Vršnak B, Žic T, Falkenberg TV, Möstl C, Vennerstrom S, Vrbanec D (2010) The role of aerodynamic drag in propagation of interplanetary coronal mass ejections. Astron Astrophys 512:A43. https://doi. org/10.1051/0004-6361/200913482

Vršnak B, Žic T, Vrbanec D, Temmer M, Rollett T, Möstl C, Veronig A, Čalogović J, Dumbović M, Lulić S, Moon YJ, Shanmugaraju A (2013) Propagation of interplanetary coronal mass ejections: the drag-based model. Sol Phys 285(1-2):295-315. https://doi.org/10.1007/s11207-012-0035-4

Vršnak B, Amerstorfer T, Dumbović M, Leitner M, Veronig AM, Temmer M, Möstl C, Amerstorfer UV, Farrugia CJ, Galvin AB (2019) Heliospheric evolution of magnetic clouds. Astrophys J 877(2):77. https://doi.org/10.3847/1538-4357/ab190a. arXiv:1904.08266 [astro-ph.SR]

Wagner WJ, Wagner JJ (1984) Coronal mass ejection recurrence studies indicating global activity and local suppression. Astron Astrophys 133(2):288-292

Wallace S, Arge CN, Pattichis M, Hock-Mysliwiec RA, Henney CJ (2019) Estimating total open heliospheric magnetic flux. Sol Phys 294(2):19. https://doi.org/10.1007/s11207-019-1402-1arXiv: 1903.12613 [astro-ph.SR]

Wang Y, Zhang J (2007) A comparative study between eruptive X-class flares associated with coronal mass ejections and confined X-class flares. Astrophys J 665(2):1428-1438. https://doi.org/10.1086/ 519765. arXiv:0808.2976 [astro-ph]

Wang Y, Shen C, Wang S, Ye P (2004) Deflection of coronal mass ejection in the interplanetary medium. Sol Phys 222(2):329-343. https://doi.org/10.1023/B:SOLA.0000043576.21942.aa

Wang Y, Wang B, Shen C, Shen F, Lugaz N (2014) Deflected propagation of a coronal mass ejection from the corona to interplanetary space. J Geophys Res 119(7):5117-5132. https://doi.org/10.1002/ 2013JA019537. arXiv:1406.4684 [physics.space-ph]

Wang YM (2013) On the strength of the hemispheric rule and the origin of active-region helicity. Astrophys J Lett 775(2):L46. https://doi.org/10.1088/2041-8205/775/2/L46

Wang YM, Sheeley NR, Socker DG, Howard RA, Rich NB (2000) The dynamical nature of coronal streamers. J Geophys Res 105(A11):25133-25142. https://doi.org/10.1029/2000JA000149

Wang YM, Ye PZ, Wang S (2003) Multiple magnetic clouds: several examples during March-April 2001. J Geophys Res 108(A10):1370. https://doi.org/10.1029/2003JA009850

Warmuth A (2015) Large-scale globally propagating coronal waves. Living Rev Sol Phys 12:3. https:// doi.org/10.1007//rsp-2015-3

Watari S (2018) Intense geomagnetic storms associated with coronal holes under the weak solar-wind conditions of cycle 24. Sol Phys 293(2):23. https://doi.org/10.1007/s11207-018-1248-y

Webb DF, Howard TA (2012) Coronal mass ejections: observations. Living Rev Sol Phys 9:3. https://doi. org/10.12942/lrsp-2012-3

Webb DF, Cliver EW, Crooker NU, St Cyr OC, Thompson BJ (2000) Relationship of halo coronal mass ejections, magnetic clouds, and magnetic storms. J Geophys Res 105(A4):7491-7508. https://doi. org/10.1029/1999JA000275

Welsch BT (2018) Flux accretion and coronal mass ejection dynamics. Sol Phys 293(7):113. https://doi. org/10.1007/s11207-018-1329-y. arXiv:1701.09082 [astro-ph.SR]

Wenzel KP, Marsden RG, Page DE, Smith EJ (1992) The Ulysses mission. Astron Astrophys Suppl 92:207

Werner ALE, Yordanova E, Dimmock AP, Temmer M (2019) Modeling the multiple CME interaction event on 6-9 September 2017 with WSA-ENLIL+Cone. Space Weather. https://doi.org/10.1029/ 2018SW001993

Wiegelmann T, Thalmann JK, Solanki SK (2014) The magnetic field in the solar atmosphere. Astron Astrophys Rev 22:78. https://doi.org/10.1007/s00159-014-0078-7. arXiv:1410.4214 [astro-ph.SR]

Wilcox JM, Hoeksema JT, Scherrer PH (1980) Origin of the warped heliospheric current sheet. Science 209:603-605. https://doi.org/10.1126/science.209.4456.603 
Wimmer-Schweingruber RF, von Steiger R, Paerli R (1997) Solar wind stream interfaces in corotating interaction regions: SWICS/Ulysses results. J Geophys Res 102(A8):17407-17418. https://oi.org/ 10.1029/97JA00951

Wood BE, Howard RA, Thernisien A, Plunkett SP, Socker DG (2009) Reconstructing the 3D morphology of the 17 May 2008 CME. Sol Phys 259(1-2):163-178. https://doi.org/10.1007/s11207-009-9391-0

Wood BE, Howard RA, Thernisien A, Socker DG (2010) The three-dimensional morphology of a corotating interaction region in the inner heliosphere. Astrophys J Lett 708(2):L89-L94. https://doi. org/10.1088/2041-8205/708/2/L89

Wu CC, Lepping RP (2002) Effect of solar wind velocity on magnetic cloud-associated magnetic storm intensity. J Geophys Res 107(A11):1346. https://doi.org/10.1029/2002JA009396

Xie H, Ofman L, Lawrence G (2004) Cone model for halo CMEs: application to space weather forecasting. J Geophys Res 109(A3):A03109. https://doi.org/10.1029/2003JA010226

Xie H, Mäkelä P, St Cyr OC, Gopalswamy N (2017) Comparison of the coronal mass ejection shock acceleration of three widespread SEP events during solar cycle 24. J Geophys Res 122(7):7021-7041. https://doi.org/10.1002/2017JA024218

Xie Y, Wei F, Feng X, Zhong D (2006) Prediction test for the two extremely strong solar storms in October 2003. Sol Phys 234(2):363-377. https://doi.org/10.1007/s11207-006-0091-8

Xu F, Borovsky JE (2015) A new four-plasma categorization scheme for the solar wind. J Geophys Res 120(1):70-100. https://doi.org/10.1002/2014JA020412

Yashiro S, Akiyama S, Gopalswamy N, Howard RA (2006) Different power-law indices in the frequency distributions of flares with and without coronal mass ejections. Astrophys J Lett 650(2):L143-L146. https://doi.org/10.1086/508876arXiv:astro-ph/0609197 [astro-ph]

Yermolaev YI, Nikolaeva NS, Lodkina IG, Yermolaev MY (2009) Catalog of large-scale solar wind phenomena during 1976-2000. Cosmic Res 47(2):81-94. https://doi.org/10.1134/ S0010952509020014

Yurchyshyn V, Abramenko V, Tripathi D (2009) Rotation of white-light coronal mass ejection structures as inferred from LASCO coronagraph. Astrophys J 705(1):426-435. https://doi.org/10.1088/0004$637 \mathrm{X} / 705 / 1 / 426$

Yurchyshyn VB, Wang H, Goode PR, Deng Y (2001) Orientation of the magnetic fields in interplanetary flux ropes and solar filaments. Astrophys J 563(1):381-388. https://doi.org/10.1086/323778

Zhang J, Dere KP (2006) A statistical study of main and residual accelerations of coronal mass ejections. Astrophys J 649:1100-1109. https://doi.org/10.1086/506903

Zhang J, Dere KP, Howard RA, Kundu MR, White SM (2001) On the temporal relationship between coronal mass ejections and flares. Astrophys J 559(1):452-462. https://doi.org/10.1086/322405

Zhang J, Dere KP, Howard RA, Vourlidas A (2004) A Study of the kinematic evolution of coronal mass ejections. Astrophys J 604(1):420-432. https://doi.org/10.1086/381725

Zhang J, Blanco-Cano X, Nitta N, Srivastava N, Mandrini CH (2018) Editorial: Earth-affecting solar transients. Sol Phys 293(5):80. https://doi.org/10.1007/s11207-018-1302-9

Zhao L, Zurbuchen TH, Fisk LA (2009) Global distribution of the solar wind during solar cycle 23: ACE observations. Geophys Res Lett 36(14):L14104. https://doi.org/10.1029/2009GL039181

Žic T, Vršnak B, Temmer M, Jacobs C (2008) Cylindrical and spherical pistons as drivers of MHD shocks. Sol Phys. https://doi.org/10.1007/s11207-008-9173-0

Zucca P, Morosan DE, Rouillard AP, Fallows R, Gallagher PT, Magdalenic J, Klein KL, Mann G, Vocks C, Carley EP, Bisi MM, Kontar EP, Rothkaehl H, Dabrowski B, Krankowski A, Anderson J, Asgekar A, Bell ME, Bentum MJ, Best P, Blaauw R, Breitling F, Broderick JW, Brouw WN, Brüggen M, Butcher HR, Ciardi B, de Geus E, Deller A, Duscha S, Eislöffel J, Garrett MA, Grießmeier JM, Gunst AW, Heald G, Hoeft M, Hörandel J, Iacobelli M, Juette E, Karastergiou A, van Leeuwen J, McKay-Bukowski D, Mulder H, Munk H, Nelles A, Orru E, Paas H, Pand ey VN, Pekal R, Pizzo R, Polatidis AG, Reich W, Rowlinson A, Schwarz DJ, Shulevski A, Sluman J, Smirnov O, Sobey C, Soida M, Thoudam S, Toribio MC, Vermeulen R, van Weeren RJ, Wucknitz O, Zarka P (2018) Shock location and CME 3D reconstruction of a solar type II radio burst with LOFAR. Astron Astrophys 615:A89. https://doi.org/10.1051/0004-6361/201732308. arXiv:1804. 01025 [astro-ph.SR]

Publisher's Note Springer Nature remains neutral with regard to jurisdictional claims in published maps and institutional affiliations. 